\title{
Irreversible Investment in Stochastically Cyclical Markets*
}

\author{
Francisco Ruiz-Aliseda \\ Universitat Pompeu Fabra
}

\author{
Jianjun $\mathrm{Wu}$ \\ University of Arizona
}

March 6, 2007

\begin{abstract}
This paper presents a new framework for studying irreversible (dis)investment when a market follows a random number of random-length cycles (such as a high-tech product market). It is assumed that a firm facing such market evolution is always unsure about whether the current cycle is the last one, although it can update its beliefs about the probability of facing a permanent decline by observing that no further growth phase arrives. We show that the existence of regime shifts in fluctuating markets suffices for an option value of waiting to (dis)invest to arise, and we provide a marginal interpretation of the optimal (dis)investment policies, absent in the real options literature. The paper also shows that, despite the stochastic process of the underlying variable has a continuous sample path, the discreteness in the regime changes implies that the sample path of the firm's value experiences jumps whenever the regime switches all of a sudden, irrespective of whether the firm is active or not.
\end{abstract}

Keywords: Real Options, Regime-Switching, Bad News Principle, Signal Extraction Problem, Entry and Exit, Industry Life Cycles.

\footnotetext{
*Corresponding author: Jianjun Wu, Department of Economics, University of Arizona, Tucson, AZ, 85721-0108. E-mails: fran.ruiz@upf.edu; jwu@eller.arizona.edu. The authors thank Rabah Amir, David Besanko, James Dana, Janice Eberly, Kathleen Hagerty, Frank Riedel, Mark Satterthwaite, Daniel Spulber for helpful conversations, as well as seminar participants at the University of Arizona and the University of Bonn for their comments, although the usual disclaimer applies. Francisco Ruiz-Aliseda acknowledges financial support from Fundación la Caixa.
} 


\section{Introduction}

Numerous products such as pharmaceuticals, electronics, or more generally high-tech goods, are characterized by a temporal evolution of demand that alternates between periods of growth and decline at random times. An additional feature of high-tech products is that they eventually disappear, replaced by superior substitutes, ${ }^{1}$ although it is actually rather hard to predict whether the introduction of an innovation will lead to a permanent decay in the demand for an established product. The case of floppy disk drives for computers is especially illuminating in this respect. Such drives dominated the market from the mideighties to the late nineties, and they successfully survived the challenge posed by several innovations such as zip drives (and a few others less known), which, despite a promising start, simply led to a transitory decline in demand for floppy drives. The irreversible decline in the demand for floppy drives had to await until the arrival of USB and CD-ROM/DVDROM drives, whose introduction led to the end of a reign of around fifteen years. ${ }^{2}$ This example suggests that when the decline phase of a high-tech industry starts, established firms (or potential entrants) are unsure about whether a sharp decline in demand is going to be temporary or permanent. However, a firm can use the length of such a phase as a signal to update its beliefs about the probability that the downturn in demand is permanent. In this sense, the longer a decline phase, the less likely that a firm perceives the demand decline as a transitory shock.

Investment decisions in high-tech industries must certainly account for such stochastically cyclical evolution of important variables such as market demand (or profit), especially when the degree of specificity of assets in which to invest is high. For these industries, the dynamics of the underlying state variable can be better represented by means of a regime-switching stochastic process rather than the standard diffusion process traditionally employed by the real options literature. ${ }^{3}$ For instance, there is no way to explicitly model growth and decline phases - i.e., the regimes of the process - using a single Geometric Brownian Motion (GBM). Recently, a few papers such as Drifill, Raybaudi and Sola (2003) or Guo, Miao and Morellec (2005) have addressed this issue by introducing models of investment behavior in which the market regime switches at random dates. In particular, these papers propose a perpetual

\footnotetext{
${ }^{1}$ See Adner and Zemsky (2005) for a discussion and a formalization of how gradual competition by "disruptive technologies" can sometimes displace established technologies, leading to their abandoning (or forcing them to serve a residual market niche).

${ }^{2}$ As pointed out by McAfee (2002, p. 102), it is worth remarking that "often the decline of one industry is caused by the growth of another, closely related industry."

${ }^{3}$ Two classic papers on real options theory are McDonald and Siegel (1986) and Dixit (1989). Pindyck (1991) or Dixit and Pindyck (1994) provide excellent surveys of some of the most relevant theoretical developments. See also the recent work by Riedel and Su (2007) for an elegant general approach to irreversible investment under uncertainty.
} 
evolution of the market demand such that it alternates between growth and decline phases at uncertain times. These models are especially well suited for analyzing (dis)investment decisions in industries that are highly sensitive to business cycle movements. Yet, they do not capture several properties that characterize the temporal evolution of demand in hightech industries, in which perpetual alternation of regimes generally does not take place due to the very own nature of such industries. At some point, demand for high-tech products starts an irreversible decline, even if it is reasonable to suppose that such decline is difficult to forecast, as illustrated by the unexpectedly long life of floppy disk drives.

In this paper, we introduce a piecewise deterministic process in continuous time in which the regime switches at random dates, but only a random number of times. Our aim is to provide a stylized model for a high-tech industry whose profit/demand follows a random number of random-length cycles (where each cycle is characterized by a growth and a decline phase). In addition to this dual source of uncertainty, economic agents face a simple filtering problem in that they cannot observe the realized number of regime shifts, that is, agents are always unsure about whether the decline phase of the current cycle is permanent or not. However, once a decline phase starts, a firm can revise its beliefs about the probability that the market decays forever as no growth phase arrives. Our main purpose is to understand general properties of this type of regime-switching models of market evolution, as well as study their implications for (dis)investment decisions in high-tech industries. For this reason, we first characterize some properties of the stochastic process that we introduce, and then apply them to the analysis and characterization of a single agent's optimal entry and exit behavior.

A novel contribution of this paper is to show that, in fluctuating market environments, the possibility of regime switching suffices for an option value of waiting to (dis)invest to arise. ${ }^{4}$ Because the length of growth and decline phases is random, a firm has an incentive to wait and continuously update its information about the duration of the current phase without making any irreversible decision, and at the same time it can capitalize on favorable market evolutions. ${ }^{5}$ The existence of an option value of delaying (dis)investment in regime-switching

\footnotetext{
${ }^{4}$ Our notion of fluctuating market evolution refers to the idea that markets grow and decline over time. However, even if the market is assumed to remain stable at a certain level, a continuously changing state variable can arise by allowing a firm to improve its payoff if it discovers a certain state of the world. If the realized state can be discovered only by exerting an a priori unknown amount of investment effort, then beliefs continuously change over time as an agent invests, and there can be an option value of investing in (time-consuming) experimentation even if the market remains at the same level. Recent pieces of work building on this idea are the game-theoretic papers by Décamps and Mariotti (2004) or Keller, Rady and Cripps (2005), or the paper on entrepreneurship by Miao and Wang (2006). See Lucas (1971) for a pioneering analysis.

${ }^{5}$ In the case of a declining market, the firm also incorporates the change in its assessment about the transitory character of the current decline phase into its information updating, which affects option values,
} 
models of fluctuating market evolution has relevant conceptual implications for the theory of real options. This theory lacks a marginal interpretation of optimal (dis)investment policies, which probably explains to a large extent why both scholars and practitioners find it hardly intuitive. Another major contribution of this paper is to provide a marginal interpretation that is linked to the "bad news principle of irreversible investment" spelled out by Bernanke (1983), which in our view is the conceptual pillar of real options theory. ${ }^{6}$ According to this principle, a firm that slightly delays investment when there is fully ongoing uncertainty should only care about the arrival of bad news and their adverse effect on payoffs. ${ }^{7}$

In the light of the "bad news principle," the optimal entry policy lends itself to the following marginal interpretation. On the one hand, slightly delaying entry into a growing market that will stop expanding after some random time is marginally valuable for two reasons. First, the firm can save part of its investment cost. Second, there is a marginal option value of waiting to invest because the firm avoids making a wrong entry decision with some probability. In particular, a slight delay allows the firm to avoid the adverse effect of a sudden regime shift — this is the "bad news"—, something which happens with positive probability. As a result, the marginal option value of waiting to invest is measured by the expected loss in the project's NPV that is avoided by waiting. On the other hand, delaying investment is costly for a firm in that it entails forgoing a profit flow, ${ }^{8}$ so optimal entry behavior must balance the marginal cost and the marginal benefit of deferring investment.

The marginal interpretation of the optimal exit policy in a declining market that may stop declining after some random time is similar in spirit. ${ }^{9}$ On the one hand, a slight delay in exit is (marginally) costly because the firm forgoes earning interest on the asset's outside value. On the other hand, there are two reasons why delaying exit is valuable. First, remaining a little bit longer in the market allows the firm to extend the period of profit harvesting. Second, deferring exit is also valuable because there is a marginal option value of waiting to disinvest, which again arises due to the possible arrival of bad news - and the start of a growth phase is bad news for an exit decision in a declining market. With some probability,

but does not dissipate them.

${ }^{6}$ Actually, a marginal interpretation of investment policies can be found in the influential paper by Abel and Eberly (1996). However, their interpretation is linked to the Jorgensonian user cost of capital rather than Bernanke's (1983) "bad news principle of irreversible investment."

${ }^{7}$ The point is that an irreversible decision is costly in that it can be regretted ex post, which can happen only if bad news have arrived. As a direct consequence, a firm should consider only the effect of the arrival of bad news (and not good news) when contemplating a delay in the execution of an irreversible decision.

${ }^{8}$ In some sense, waiting to invest amounts to exercising an option to learn about future market conditions without making any irreversible decision. The cost of exercising such an option is the profit flow forgone by delaying investment.

${ }^{9}$ It is worth pointing out that the paper shows that the firm finds it optimal to exit only if the market is declining. Similarly, the firm finds it optimal to enter only if the market is growing. 
the regime may shift and the market may start growing, so waiting allows the firm to avoid making a poor exit decision in such a case, since remaining active in a growing market while keeping the option to exit alive can be shown to be more valuable than seizing the outside option. $^{10}$ It is important to note that the marginal option value of waiting to disinvest arises in a market environment subject to fluctuations because the regime shifts at random dates. In particular, it does not arise because the regime switches just a random number of times. In fact, based on the insights provided by the "bad news principle," it holds that the randomness in the number of regime switches affects only the probability of arrival of bad news, ${ }^{11}$ and hence creates an incentive to exit at higher demand/profit levels because it lowers the marginal option value of waiting to disinvest. In turn, the unobservability of the realized number of regime shifts affects only the probability of regime shift that is perceived by the firm based on the length of time elapsed since the market last switched. Because the firm becomes pessimistic about the arrival of bad news as no growth phase starts, another application of the "bad news principle" yields that the level of demand/profit at which the firm finds it optimal to disinvest is higher the earlier the decline phase started.

Another contribution of this paper is to draw implications for firm valuation of regimeswitching models of market evolution. By construction, the stochastic process that we examine has a continuous sample path despite its discrete number of regimes. ${ }^{12}$ However, we show that the sample path of the value of a (dis)investment opportunity experiences jumps whenever the regime switches all of a sudden. In particular, firm value jumps upwards (downwards) whenever a growth (decline) phase starts, so imperceptible changes in the profit flow collected by the firm may be accompanied by significant falls or rises in firm value due to the discreteness of the regime shifts. ${ }^{13}$ Such prediction is in stark contrast to that of conventional real options models, which do not predict jumps in the value of a firm, whether active or not, when the stochastic process of the underlying variable has a continuous sample path. In addition, our paper gives a rationale for using jump processes in the valuation of high-tech firms' stock prices based on a regime-switching stochastic process for the underlying state variable.

Our paper contributes to the recent real options literature based on regime-switching

\footnotetext{
${ }^{10}$ Of course, it need not be true for other (suboptimal) exit policies that seizing the outside option is less valuable than remaining active in a growing market while keeping the option to exit alive.

${ }^{11}$ Recall that the start of a growth phase constitutes bad news for an exit decision in a market that is declining.

${ }^{12}$ More precisely, because regime shifts only refer to the instantaneous growth rate of the flow profits, which preserves the continuity of the process that governs profit evolution.

${ }^{13}$ It is worth pointing out that the assumption that the market may not go through more cycles in the future with some probability, and the unobservability of this event, do not cause the jumps. Yet, they do affect their size (and the rate at which firm value decreases during a decline phase).
} 
models. The work by Drifill, Raybaudi and Sola (2003) numerically analyzes how the value of a single investment opportunity is affected by the existence of an infinite number of regime shifts. In a similar market environment, Guo, Miao and Morellec (2005) examine a firm's capital accumulation process and find the remarkable result that regime shifts may explain why investment in divisible capital can be intermittent and lumpy even if fixed adjustment costs are negligible.

The current paper differs from these papers in several respects. From a technical standpoint, these regime-switching models of investment have not paid attention to those situations in which the regime switches a random number of times, let alone to those in which the realized number of regime shifts is unobservable. As a result, their models are more adequate for markets whose evolution is highly correlated with that of the business cycle, ${ }^{14}$ whereas our framework is intended to model high-tech industries subject to market-specific fluctuations. In addition, to the best of our knowledge, we are the first in characterizing some general properties of a regime-switching stochastic process such as the (conditionally) expected stream of discounted profits harvested while the process transitions from one state to another. From a conceptual standpoint, our paper differs in that we show that the existence of regime shifts in a fluctuating market is sufficient to give rise to an option value of waiting to (dis)invest. ${ }^{15}$ Furthermore, we strengthen the conceptual foundations of real options theory by providing a marginal interpretation linked to Bernanke's (1983) "bad news principle" for both entry and exit policies, even in the complicated case in which the firm cannot observe whether the current cycle is going to be the last one. Lastly, from a predictive point of view, we show that the discreteness in regime changes leads to a discontinuous sample path for firm value although the sample path of the stochastic process for instantaneous profit is continuous. Not only do we show that the sample path is discontinuous, but also show that it jumps up (down) when a decline phase stops (starts), irrespective of whether the firm has an option to invest or disinvest.

The remainder of the paper is organized as follows. Section 2 describes the stochastic

\footnotetext{
${ }^{14}$ Some representative industries that are sensitive to macroeconomic conditions are those producing durable goods such as motor vehicles or electrical appliances (see Berman and Pfleeger 1997 for many more examples). It is worth noting that our main results apply to this type of industries as well (since it corresponds to the specific case in which the market reaches an irreversible market decline with zero probability).

${ }^{15}$ To be precise, the papers by Drifill, Raybaudi and Sola (2003) and Guo, Miao and Morellec (2005) model the temporal evolution of demand by means of a GBM together with a (discrete) switching of the drift and volatility parameters of such process at random dates. Apart from assuming a random number of regime shifts whose realization is unobservable to the decision-maker, we differ from these two papers in that we set the volatility parameters of their process equal to zero. Doing so allows us to show in a transparent manner that switches in the drift of their diffusion process are sufficient to create an option value of waiting to (dis)invest, which unveils relevant implications of regime switching models of investment that were previously hidden by the GBM assumption.
} 
process that constitutes the starting point of our analysis. Given the novelty of the process, Section 3 provides several mathematical results such as expected discount factors on the state space and present value calculations, which makes some of the results in subsequent sections quite straightforward. Section 4 and 5 respectively characterize the optimal entry and exit policies, relate them to the "bad news principle," and analyze their implications for firm valuation. Section 6 concludes. Proofs of the results can be found in two appendices.

\section{The model}

In this section we construct a stochastic process with continuous sample paths that represents the random evolution of a certain variable $\Pi$. For the sake of concreteness, $\Pi(t)$ denotes instantaneous profit at time $t$, although it could certainly represent any other variable such as demand or price of a product.

Let the dynamics of flow profits be such that $d \Pi=\alpha(t) \Pi d t$, where $\{\alpha(t), t \geq 0\}$ is a Markov chain with states $\left\{\alpha_{1}, \alpha_{2}\right\} \in \Re_{++} \times \Re_{--}$. It is assumed for convenience that the chain starts at state $\alpha_{1}$ (i.e., $\alpha(0)=\alpha_{1}$ ), while the transition probabilities of this process are as follows. On the one hand, if the chain is in state $\alpha_{1}$ at time $t \geq 0$, then the probability that it moves to state $\alpha_{2}$ between times $t$ and $t+d t$ is $\operatorname{Pr}\left(\alpha(t+d t)=\alpha_{2} \mid \alpha(t)=\alpha_{1}\right)=\lambda_{1} d t+o(d t)$. On the other hand, if the chain is in state $\alpha_{2}$ at time $t>0$, then the probability that it moves to state $\alpha_{1}$ between times $t$ and $t+d t$ is $\operatorname{Pr}\left(\alpha(t+d t)=\alpha_{1} \mid \alpha(t)=\alpha_{2}\right)=\widetilde{\Lambda} \lambda_{2} d t+o(d t)$, where $\widetilde{\Lambda}$ is a Bernoulli random variable that is independently drawn every time the chain leaves state $\alpha_{1}$. It is assumed that $\Lambda=1$ with probability $p_{0} \in[0,1]$ and $\Lambda=0$ with probability $1-p_{0} \cdot{ }^{16}$ It is also assumed that every draw is unobservable as long as the chain does not leave state $\alpha_{2} \cdot{ }^{17}$

Letting $\widetilde{\tau}_{i}$ denote the inter-arrival time of event $i \in\{1,2, \ldots\}$ (where an event is a change in the state of the chain), we have that $\left\{\widetilde{\tau}_{i}\right\}_{i=1}^{\infty}$ is a sequence of exponential random variables such that the inter-arrival times with odd (even) subscripts are exponentially distributed with rate $\lambda_{1}>0\left(\lambda_{2}>0\right) .{ }^{18}$ We define $T_{i}=T_{i-1}+\tau_{i}$, for all $i \in\{1,2, \ldots\}$, where the initial date is $T_{0}=0$, and we refer to each $T_{i}$ as a (realized) switching date. Because of the i.i.d. assumption on the Bernoulli random variable $\widetilde{\Lambda}$, only the first $2 \widetilde{n}-1$ elements of sequence

\footnotetext{
${ }^{16}$ When $p_{0}=1$, this process is the continuous-time limit of the stochastic process put forward by Bagwell and Staiger (1997) in their analysis of collusive behavior over the business cycle.

${ }^{17}$ Equivalently, we assume that state $\alpha_{1}$ is always transient, whereas state $\alpha_{2}$ can be either transient or absorbing. In particular, such a state becomes absorbing once the chain has been $\widetilde{n}$ times in state $\alpha_{1}$, where $\widetilde{n}$ is a geometrically distributed random variable with parameter $1-p_{0}$ whose draw is unobservable. (Hence, $\operatorname{Pr}(\widetilde{n}=n)=\left(1-p_{0}\right) p_{0}^{n-1}$ for $n \in\{1,2, \ldots\}$.) If state $\alpha_{2}$ has not yet become absorbing and rather is transient, then we assume that the transition probability is $\operatorname{Pr}\left(\alpha(t+d t)=\alpha_{1} \mid \alpha(t)=\alpha_{2}\right)=\lambda_{2} d t+o(d t)$.

${ }^{18}$ The rates $\lambda_{1}$ and $\lambda_{2}$ are allowed to be different.
} 
$\left\{\widetilde{\tau}_{i}\right\}_{i=1}^{\infty}$ matter, where $\widetilde{n}$ is a geometrically distributed random variable with parameter $1-p_{0}$. As mentioned earlier, the draw of such probability distribution is never observed (although it is perfectly learnt ex post if the chain leaves state $\alpha_{2}$, which can happen only if $\Lambda=1$ was drawn when the process left state $\alpha_{1}$; otherwise, learning of the realization is imperfect).

Figure 1 shows two sample paths of the process we have described. As seen in the figure, $\Pi(t)$ grows exponentially at rate $\alpha_{1}>0$ during the random length period $\left(T_{i-1}, T_{i}\right)$ $(i=1,3, \ldots)$, and decreases exponentially at rate $\alpha_{2}<0$ during $\left(T_{i-1}, T_{i}\right)(i=2,4, \ldots)$, but after some cycles it reaches a downturn that never ends. The sample path is assumed to be continuous because $\lim _{t \uparrow T_{i}} \Pi(t)=\lim _{t \downarrow T_{i}} \Pi(t)$, although the path will exhibit a kink at any realized switching date $T_{i}(i=1,2, \ldots)$, and it will be almost everywhere differentiable. Lastly, it holds that $\Pi(t)>0$ for any $t \geq 0$ and any set of realizations of the random variables involved because we assume that $\Pi(0)>0$.

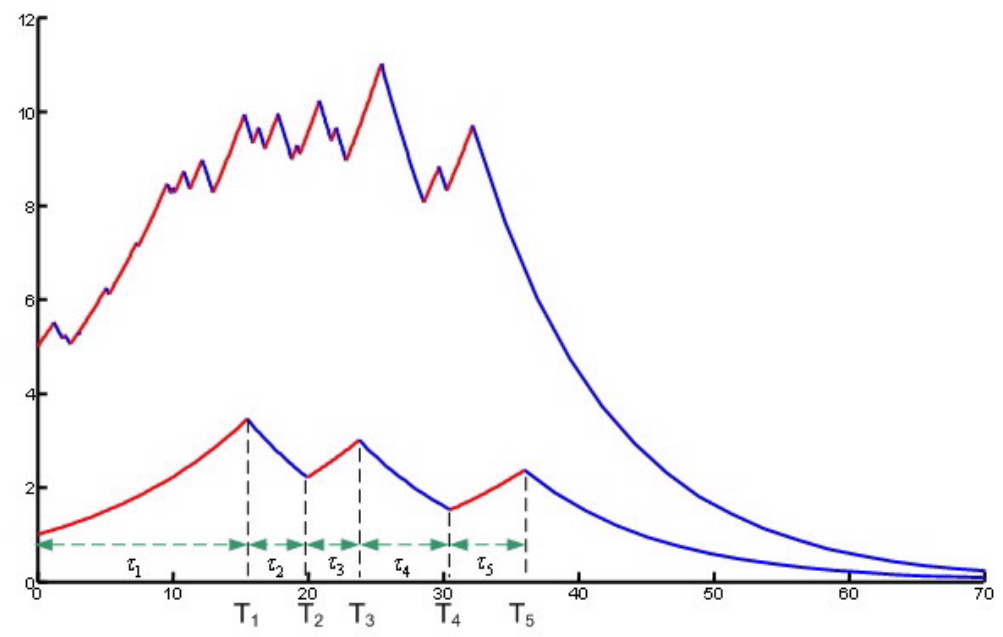

Figure 1: Two sample paths

We say that the process (or, more fundamentally, market) is in a growth phase when it is characterized by a positive instantaneous growth rate; otherwise, we say that the process is in a decline phase. Because of the i.i.d. exponential random variables, the current state of the market is clearly summarized by the current level of the flow profit and the current type of phase through which the market is going. In addition, if the market is in a decline phase, then there exists a simple signal extraction problem because the transition probability is unknown. Given that $\widetilde{\Lambda}$ is an i.i.d. Bernoulli random variable drawn every time the market switches to decline, the level at which the last decline phase started (together with the current level of the market) is another state variable, since such information is a sufficient statistic for forming (posterior) beliefs about the probability that the current declining phase 
is not the last one.

This paper is concerned with (dis)investment decisions given the random temporal evolution of a variable that determines the profitability of a (dis)investment opportunity. Our points can be conveyed more efficiently by focusing on a single decision-maker (as in Dixit and Pindyck 1994), even if further applications may be based on more agents. In particular, the paper studies a single firm's decisions to enter and exit a market, each in isolation, ${ }^{19}$ given that the flow of profits made by the firm if active in the market follows the stochastic process previously described. Specifically, at each date, the firm does not know when the next upturns or downturns will happen, although it knows the current level of the profit flow, whether the cycle is growing or declining and at what level the market started declining (if applicable). The firm also observes realized switching dates immediately once they arrive. The firm is assumed to be risk-neutral and uses a constant discount rate $r>0$. If the firm enters the market, then it is assumed to incur a positive sunk cost $K$ and in return starts operating immediately (i.e., there is no time-to-build). Similarly, if the firm exits the market, then it is assumed to recover a non-negative value $S$, which can be the salvage or redeployment value of the asset.

\section{Mathematical preliminaries}

In this section we derive some relevant mathematical results regarding the stochastic process defined in Section 2. They are useful properties for working on the state space of the process and they will be used in Sections 4 and 5. Throughout, we denote the current level of the profit flow by $\pi_{0},{ }^{20}$ and relegate proofs to Appendix A.

We first deal with the manner in which the firm updates its beliefs about the probability that the current cycle is the last one. Clearly, when the market is growing, the firm does not learn anything about whether the next decline phase is going to be the last one or not. However, once the market has started declining, the firm does not know whether the decline phase that has just begun is the last one, although it can revise its prior belief about whether the current downturn is the last one or not by observing that no growth phase arrives. Intuitively, the firm becomes more pessimistic as more time elapses without a growth phase arriving. The following result based on Bayes' rule corroborates this intuition, but on the state space rather than the time space:

\footnotetext{
${ }^{19}$ One can study combined entry and exit as in Dixit (1989), but the results yield little insights additional to those we derive in this paper.

${ }^{20}$ We also use "upperbar" and "lowerbar" notation to respectively denote growth and decline phases. The only exception is $\bar{\pi}$, which is used to denote the state (i.e., level of the flow profit) at which the current decline phase has started.
} 
Lemma 1 Suppose that the process is in a decline phase that started at level $\bar{\pi}$ and that the current state is $\pi_{0} \leq \bar{\pi}$. Then the posterior probability that the decline phase is not going to last forever is $p\left(\pi_{0} \mid \bar{\pi}\right)=\frac{p_{0}\left(\bar{\pi} / \pi_{0}\right)^{\frac{\lambda_{2}}{\alpha_{2}}}}{p_{0}\left(\bar{\pi} / \pi_{0}\right)^{\frac{\lambda_{2}}{\alpha_{2}}}+\left(1-p_{0}\right)}$. Such posterior probability is increasing in $\pi_{0}$

Figure 2 uses Lemma 1 to illustrate the sample path of the posterior belief that the current cycle is the last one (denoted by $P(t)$ and plotted in the bottom graph) that is induced by a sample path of the stochastic process for profits (see top graph). It can be observed that the firm becomes pessimistic during a decline phase as long as no growth phase arrives. Also, once the market starts an irreversible decline (which in this case happens in the third cycle), the posterior belief that the decline phase is transitory converges to zero as the period of time elapsed since the last switching date grows large.

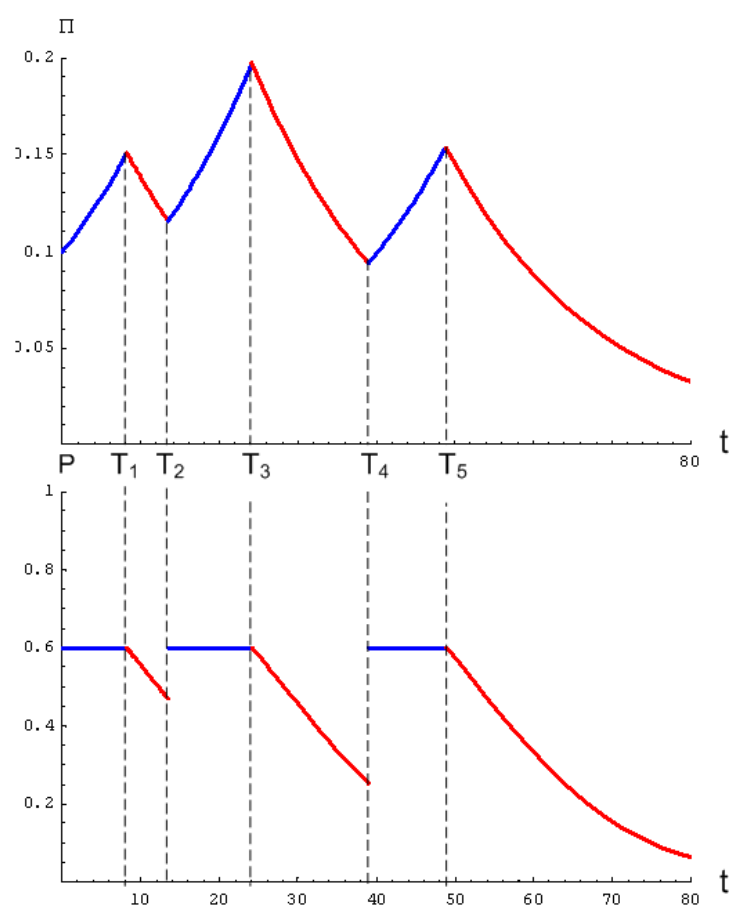

Figure 2: Sample path of $\Pi(t)$ and induced sample path of $P(t)$ when $\alpha_{1}=0.05=-\alpha_{2}, \lambda_{1}=$ $\lambda_{2}=0.1$ and $p_{0}=0.6$.

Our next result deals with the expected values of discounted streams of flow profits, but before proceeding to computing them, it is useful to introduce some notation. In particular, let

$$
\rho_{1} \equiv \frac{\left(r-\alpha_{2}\right)\left(r+\lambda_{2}-\alpha_{2}\right)+\lambda_{1}\left[r+\lambda_{2}\left(1-p_{0}\right)-\alpha_{2}\right]}{\left(r-\alpha_{2}\right)\left[\left(r+\lambda_{1}-\alpha_{1}\right)\left(r+\lambda_{2}-\alpha_{2}\right)-p_{0} \lambda_{1} \lambda_{2}\right]}
$$


and

$$
\rho_{2}\left(\pi_{0}, \bar{\pi}\right) \equiv \frac{\left(r-\alpha_{2}\right)\left(r+\lambda_{1}-\alpha_{1}\right)+\lambda_{2}\left[r+\lambda_{1}\left(1-p_{0}\right)-\alpha_{1}\right]+\lambda_{2}\left(\alpha_{1}-\alpha_{2}\right) p\left(\pi_{0} \mid \bar{\pi}\right)}{\left(r-\alpha_{2}\right)\left[\left(r+\lambda_{1}-\alpha_{1}\right)\left(r+\lambda_{2}-\alpha_{2}\right)-p_{0} \lambda_{1} \lambda_{2}\right]},
$$

and assume that

$$
\left(r+\lambda_{1}-\alpha_{1}\right)\left(r+\lambda_{2}-\alpha_{2}\right)>p_{0} \lambda_{1} \lambda_{2}
$$

in order to have an economically meaningful setting (otherwise, streams of discounted profits fail to be integrable). A necessary condition for (1) to hold is that $r+\lambda_{1}>\alpha_{1}$.

We now deal with the expected stream of discounted profits if the firm is active in the market forever given the current state $\pi_{0}$. To this end, let $\overline{\mathcal{E}}\left(\pi_{0}, \pi^{*}\right)$ denote the expected stream of discounted profits while the process transitions from $\pi_{0}$ until it first hits $\pi^{*} \leq \pi_{0}$ from above, given that the process is in a growth phase and that the current state is $\pi_{0}$. Similarly, let $\underline{\mathcal{E}}\left(\pi_{0}, \pi^{*} \mid \bar{\pi}\right)$ denote the expected stream of discounted profits while the process transitions from $\pi_{0}$ until it first hits $\pi^{*}<\pi_{0}$ from above, given that the process is in a decline phase that started at level $\bar{\pi}$ and that the current state is $\pi_{0} \leq \bar{\pi}$. Letting $\pi^{*}=0$ yields that the expected stream of discounted profits if the firm is active forever as a function of the corresponding state variables takes the following form:

Theorem 1 Suppose that the firm is operating at $\pi_{0}$ and is active forever. Then the expected stream of discounted profits is $\overline{\mathcal{E}}\left(\pi_{0}, 0\right)=\rho_{1} \pi_{0}$ if the process is in a growth phase, and $\underline{\mathcal{E}}\left(\pi_{0}, 0 \mid \bar{\pi}\right)=\rho_{2}\left(\pi_{0}, \bar{\pi}\right) \pi_{0}$ if the process is in a decline phase that started at $\bar{\pi}$ and has not stopped declining since then. Furthermore, $\overline{\mathcal{E}}\left(\pi_{0}, 0\right)>\underline{\mathcal{E}}\left(\pi_{0}, 0 \mid \bar{\pi}\right)>0$.

In order to properly discount (one-shot) payoffs - such as investment costs or scrap values - attained when the process reaches a certain state, it is also necessary to derive the (conditionally) expected discounted value of a claim to a dollar at the first date at which the process hits a certain state from above or below. ${ }^{21}$ Such value is commonly referred to as "the expected discount factor," and we stick to this terminology in the remainder of the paper. Letting

$$
\Delta \equiv\left[\alpha_{1}\left(\lambda_{2}+r\right)-\alpha_{2}\left(\lambda_{1}+r\right)\right]^{2}+4 p_{0} \alpha_{1} \alpha_{2} \lambda_{1} \lambda_{2}>0
$$

we can derive the expected discount factor to be used when the dollar is achieved the first time the process reaches a certain state from below:

Theorem 2 (i) Suppose that the process is in a growth phase and that the current state is $\pi_{0}$. Then the expected discounted value of a claim to a dollar when the process first hits

\footnotetext{
${ }^{21}$ Expectations are conditional upon the current state being $\pi_{0}$ and upon whether the process is growing or not. In the latter case, it is also conditional on the state $\bar{\pi} \geq \pi_{0}$ at which the market started its decline.
} 
$\pi^{*}>\pi_{0}$ from below is

$$
\bar{\varphi}_{1}\left(\pi_{0}, \pi^{*}\right)=\left(\frac{\pi_{0}}{\pi^{*}}\right)^{\beta_{1}},
$$

where $\beta_{1} \equiv \frac{\alpha_{1}\left(r+\lambda_{2}\right)+\alpha_{2}\left(r+\lambda_{1}\right)-\sqrt{\Delta}}{2 \alpha_{1} \alpha_{2}}>1$.

(ii) Suppose that the process is in a decline phase that started at level $\bar{\pi}$ and that the current state is $\pi_{0} \leq \bar{\pi}$. Then the expected discounted value of a claim to a dollar when the process first hits $\pi^{*} \geq \pi_{0}$ from below is

$$
\underline{\varphi}_{1}\left(\pi_{0}, \pi^{*} \mid \bar{\pi}\right)=\frac{p\left(\pi_{0} \mid \bar{\pi}\right) \delta_{2}}{p_{0}}\left(\frac{\pi_{0}}{\pi^{*}}\right)^{\beta_{1}},
$$

where $\delta_{2} \equiv-\frac{\alpha_{1}\left(r+\lambda_{2}\right)-\alpha_{2}\left(r+\lambda_{1}\right)-\sqrt{\Delta}}{2 \alpha_{2} \lambda_{1}} \in(0,1)$.

Figure 3 provides a visual illustration of the problem. The process starts growing from $\pi_{0}$ and after several cycles first hits $\pi^{*}$ from below at the (random) first-passage time $T^{*}$. Theorem 2 shows that the expected discounted value of a claim to a dollar attained at the random time $T^{*}$ is given by $\bar{\varphi}_{1}\left(\pi_{0}, \pi^{*}\right)$.

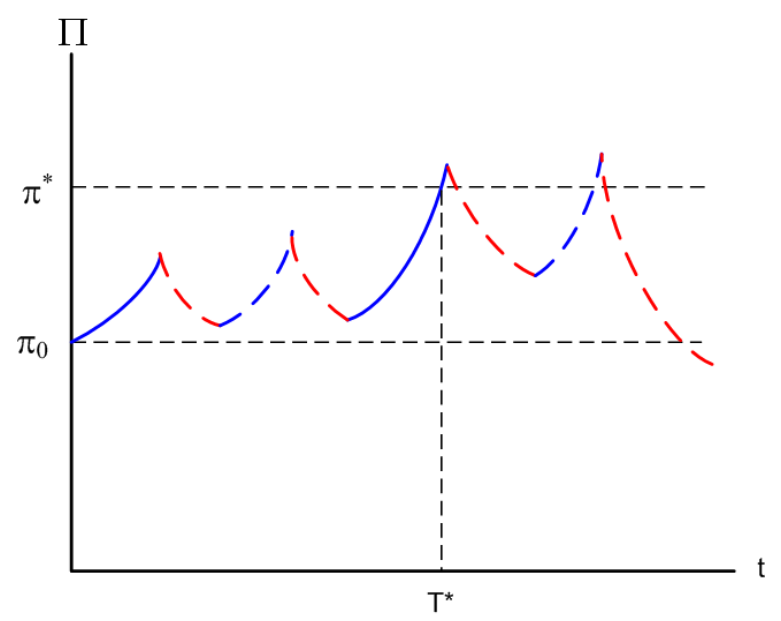

Figure 3: Expected discounted factor conditional upon the process growing at $\pi_{0}$

Theorem 2 deals with expected discount factors that are useful when discounting one-shot payoffs that are attained when the process first hits a certain state from below. Discounting of one-shot payoffs achieved when the process first hits a certain level from above requires some additional notation. In particular, letting

$$
\phi \equiv \frac{r\left(\alpha_{1}-\alpha_{2}\right)}{r\left(\alpha_{1}-\alpha_{2}\right)-\lambda_{1} \alpha_{2}\left(1-p_{0}\right)} \in(0,1),
$$


we have the parallel result to Theorem 2:

Theorem 3 (i) Suppose that the process is in a decline phase that started at level $\bar{\pi}$ and that the current state is $\pi_{0} \leq \bar{\pi}$. Then the expected discounted value of a claim to a dollar when the process first hits $\pi^{*}<\pi_{0}$ from above is

$$
\underline{\varphi}_{2}\left(\pi_{0}, \pi^{*} \mid \bar{\pi}\right)=\phi p\left(\pi_{0} \mid \bar{\pi}\right)\left(\frac{\pi_{0}}{\pi^{*}}\right)^{\beta_{2}}+\left(1-\phi p\left(\pi_{0} \mid \bar{\pi}\right)\right)\left(\frac{\pi_{0}}{\pi^{*}}\right)^{\frac{r}{\alpha_{2}}}
$$

where $\beta_{2} \equiv \frac{\alpha_{1}\left(r+\lambda_{2}\right)+\alpha_{2}\left(r+\lambda_{1}\right)+\sqrt{\Delta}}{2 \alpha_{1} \alpha_{2}}<0$.

(ii) Suppose that the process is in a growth phase and that the current state is $\pi_{0}$. Then the expected discounted value of a claim to a dollar when the process first hits $\pi^{*} \leq \pi_{0}$ from above is

$$
\bar{\varphi}_{2}\left(\pi_{0}, \pi^{*}\right)=\left(\phi+\delta_{1}-1\right)\left(\frac{\pi_{0}}{\pi^{*}}\right)^{\beta_{2}}+(1-\phi)\left(\frac{\pi_{0}}{\pi^{*}}\right)^{\frac{r}{\alpha_{2}}}
$$

where $\delta_{1} \equiv 1-\phi+\frac{\left(\lambda_{2}+r-\alpha_{2} \beta_{2}\right) \phi}{\lambda_{2}} \in(0,1)$.

This problem is represented in Figure 4, which depicts a situation in which the process starts declining at $\bar{\pi}$ and the current state is $\pi_{0}$. It can be observed that, after several cycles, the process first hits $\pi^{* *}$ from above at the (random) first-passage time $T^{* *}$. Theorem 3 shows that the expected discounted value of a claim to a dollar attained at time $T^{* *}$ is given by $\underline{\varphi}_{2}\left(\pi_{0}, \pi^{* *} \mid \bar{\pi}\right)$.

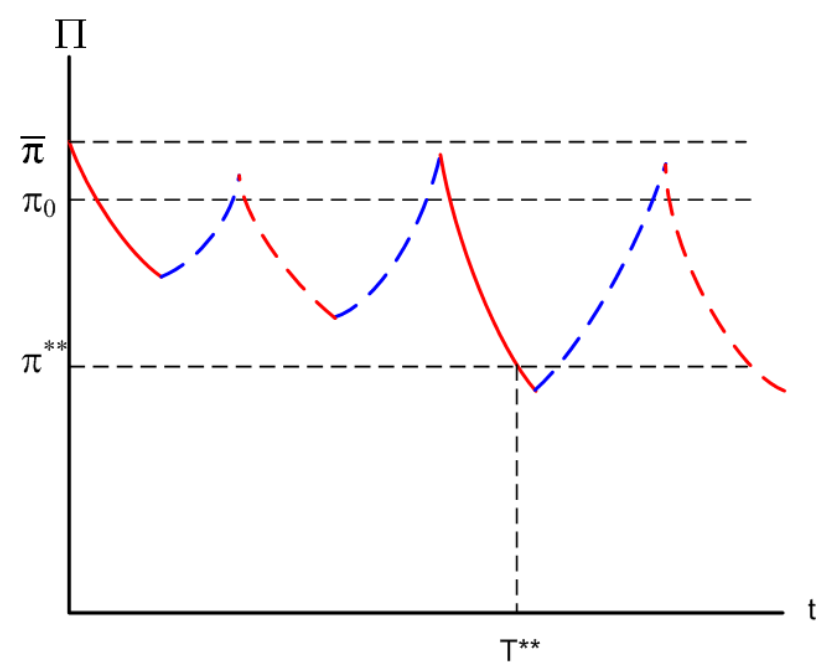

Figure 4: Expected discounted factor conditional upon the process declining at $\pi_{0}$ given that its decline started at $\bar{\pi}$

We can draw a useful corollary from the previous two theorems: 
Corollary 1 ( $i$ ) Suppose that the process is in a growth phase and that the current state is $\pi_{0}$. Then the expected discounted value of a claim to a dollar when the process first hits $\pi_{0}$ from above is $\bar{\varphi}_{2}\left(\pi_{0}, \pi_{0}\right)=\delta_{1} \in(0,1)$.

(ii) Suppose that the process is in a decline phase that started at level $\pi_{0}$ and that the current state is $\pi_{0}$. Then the expected discounted value of a claim to a dollar when the process first hits $\pi_{0}$ from below is $\underline{\varphi}_{1}\left(\pi_{0}, \pi_{0} \mid \pi_{0}\right)=\delta_{2} \in(0,1)$.

To conclude with our results in this section, notice that the expected stream of discounted profits derived in Theorem 1 is based on the hypothesis that an active firm never becomes inactive, which is relevant for completely irreversible investment decisions. Sometimes, as when the firm foresees exiting at some random time in the future, it is also necessary to compute the (conditionally) expected stream of discounted profits harvested until a certain state is first hit by the process. The following result deals with the expected discounted profit stream while transitioning from the current state to another one.

Theorem 4 (i) Suppose that the process is in a decline phase that started at level $\bar{\pi}$ and that the current state is $\pi_{0} \leq \bar{\pi}$. Then the expected stream of discounted profits while the process transitions from $\pi_{0}$ until it first hits $\pi^{*}<\pi_{0}$ from above is

$$
\underline{\mathcal{E}}\left(\pi_{0}, \pi^{*} \mid \bar{\pi}\right)=\rho_{2}\left(\pi_{0}, \bar{\pi}\right) \pi_{0}\left[1-\left(\frac{\pi_{0}}{\pi^{*}}\right)^{\beta_{2}-1}\right]-\frac{\pi_{0}\left(1-\phi p\left(\pi_{0} \mid \bar{\pi}\right)\right)}{r-\alpha_{2}}\left[\left(\frac{\pi_{0}}{\pi^{*}}\right)^{\frac{r-\alpha_{2}}{\alpha_{2}}}-\left(\frac{\pi_{0}}{\pi^{*}}\right)^{\beta_{2}-1}\right] .
$$

(ii) Suppose that the process is in a growth phase and that the current state is $\pi_{0}$. Then the expected stream of discounted profits while the process transitions from $\pi_{0}$ until it first hits $\pi^{*} \leq \pi_{0}$ from above is

$\overline{\mathcal{E}}\left(\pi_{0}, \pi^{*}\right)=\rho_{1} \pi_{0}-\frac{(1-\phi) \pi_{0}}{r-\alpha_{2}}\left(\frac{\pi_{0}}{\pi^{*}}\right)^{\frac{r-\alpha_{2}}{\alpha_{2}}}+\frac{\left(1-\delta_{1}-\phi\right) \pi_{0}}{\phi}\left(\frac{1+\lambda_{2} \rho_{1}}{r+\lambda_{2}-\alpha_{2}}-\frac{1-\phi}{r-\alpha_{2}}\right)\left(\frac{\pi_{0}}{\pi^{*}}\right)^{\beta_{2}-1}$.

Figure 4 illustrates the situation faced by the firm when computing the expected stream of discounted profits while the process transitions from $\pi_{0}$ until it first hits $\pi^{* *} \leq \pi_{0}$ from above, which happens at the first-passage time $T^{* *}$. The formula for computing such expected payoff is given by $\underline{\mathcal{E}}\left(\pi_{0}, \pi^{* *} \mid \bar{\pi}\right)$.

It is worth noting that Theorem 1 is a corollary from Theorem 4, since we have that $\underline{\mathcal{E}}\left(\pi_{0}, \pi^{*} \mid \bar{\pi}\right)=\underline{\mathcal{E}}\left(\pi_{0}, 0 \mid \bar{\pi}\right)$ and $\overline{\mathcal{E}}\left(\pi_{0}, \pi^{*}\right)=\overline{\mathcal{E}}\left(\pi_{0}, 0\right)$ for $\pi^{*}=0$. This theorem is also useful in drawing a simple but relevant result that will be used when dealing with disinvestment decisions. Letting

$$
\gamma_{1} \equiv \rho_{1}-\frac{(1-\phi)}{r-\alpha_{2}}+\frac{\left(1-\delta_{1}-\phi\right)}{\phi}\left(\frac{1+\lambda_{2} \rho_{1}}{r+\lambda_{2}-\alpha_{2}}-\frac{1-\phi}{r-\alpha_{2}}\right)
$$


so that

$$
\gamma_{1}=\frac{\rho_{2}\left[\lambda_{2}\left(1-\delta_{1}\right)+r-\alpha_{2}\right]-1}{\lambda_{2}}
$$

in the case that $p_{0}=1,{ }^{22}$ Theorem 4 leads to the following result:

Corollary 2 Suppose that the process is in a growth phase and that the current state is $\pi_{0}$. Then the expected stream of discounted profits while the process transitions from $\pi_{0}$ until it first hits $\pi_{0}$ from above is $\overline{\mathcal{E}}\left(\pi_{0}, \pi_{0}\right)=\gamma_{1} \pi_{0}$.

\section{$4 \quad$ Entry decision under uncertainty}

The purpose of this section is to characterize the main properties of optimal investment behavior and analyze its implications for firm valuation. For this reason, we assume that the firm is not initially active in a stochastically cyclical market such as the one described in Section 2. If the firm decides to undertake the investment and incur a sunk cost $K>0$, then it is assumed to operate indefinitely, i.e., the value of the outside option, $S$, equals 0 . To shorten proofs, it is also assumed throughout this section that $p_{0}=1$, although this assumption has no impact on optimal investment behavior in the unrestricted case, since the firm is never willing to enter a declining market when $p_{0}=1$ (see Lemma 2 below). The main implication is that the appeal of entering a declining market is even lower when $p_{0}<1$, since the firm becomes more and more pessimistic about the chances that the market starts another growth phase in the future as it keeps on declining. ${ }^{23}$

The stochastic process of Section 2 is Markovian and homogeneous when $p_{0}=1$, so the firm's optimal investment rule for each phase of a cycle is stationary. The firm's entry problem is even simpler because, as claimed earlier, investment does not take place while the market is declining, except for corner solutions which are ruled out to make the analysis nontrivial. This intuitive result is formally stated as follows:

Lemma 2 The firm's optimal investment policy calls for no investment while the market is declining.

Proof. See Appendix B.

To see why the lemma holds intuitively, suppose that the firm's optimal entry rule called for investment during a decline phase. Given that any profit level that is reached in a

\footnotetext{
${ }^{22}$ Since in this case $\phi=1$ and $\rho_{1}=\frac{\rho_{2}(r}{\left.+\lambda_{2}-\alpha_{2}\right)-1} \lambda_{2}$.

${ }^{23}$ In other words, continuously learning bad news about the prospects of the market while it is declining makes investment even less attractive than when the firm does not learn such bad news.
} 
declining phase must have been reached in a growth phase, it is clear that the firm could have done better by investing at the same level in the growth phase. The reason is that, in the worst-case scenario, the market would suddenly stop growing and start declining at such level, so the firm should expect to gain more if the market were growing than if it invested immediately in the downturn. This would entail a contradiction.

Therefore, Lemma 2 yields that it suffices to pay attention to phases in which the market is in growth when solving for the firm's optimal investment threshold. Thus, suppose that the market is currently growing and denote the current state of the market by $\pi_{0}$. The firm simply chooses a threshold $\pi_{E}$ such that it enters the market the first time the process hits such threshold from below. Hence, the firm solves the following optimization problem:

$$
\begin{aligned}
\max _{\pi_{E}} \bar{V}_{E}\left(\pi_{E} \mid \pi_{0}\right) & =\left[\overline{\mathcal{E}}\left(\pi_{E}, 0\right)-K\right] \bar{\varphi}_{1}\left(\pi_{0}, \pi_{E}\right) \\
& =\left(\rho_{1} \pi_{E}-K\right)\left(\frac{\pi_{0}}{\pi_{E}}\right)^{\beta_{1}}
\end{aligned}
$$

where the last equality makes use of Theorems 1 and 2 . Thus, the firm achieves an expected net payoff of $\overline{\mathcal{E}}\left(\pi_{E}, 0\right)-K$ the first time the market reaches level $\pi_{E}$ starting from state $\pi_{0}$. For this reason, $\bar{\varphi}_{1}\left(\pi_{0}, \pi_{E}\right)$ is the expected discount factor that must be used when discounting on the state space.

Because $\bar{V}_{E}\left(\pi_{E} \mid \pi_{0}\right)$ is strictly quasi-concave and

$$
\frac{\partial \bar{V}_{E}\left(\pi_{E} \mid \pi_{0}\right)}{\partial \pi_{E}}=\left[\frac{\rho_{1}\left(1-\beta_{1}\right) \pi_{E}+\beta_{1} K}{\pi_{E}}\right]\left(\frac{\pi_{0}}{\pi_{E}}\right)^{\beta_{1}}
$$

easy manipulations lead to the optimal investment threshold and the value of the investment opportunity:

Proposition 1 Suppose that the market is growing and that the firm is currently inactive. Then the firm's optimal entry rule is to invest as soon as the market reaches state

$$
\pi_{E}^{*}=\frac{1}{\rho_{1}} \frac{\beta_{1}}{\beta_{1}-1} K
$$

where $\beta_{1}=\frac{\alpha_{1}\left(r+\lambda_{2}\right)+\alpha_{2}\left(r+\lambda_{1}\right)-\sqrt{\Delta}}{2 \alpha_{1} \alpha_{2}}>1$. The value of the firm is

$$
\bar{V}_{E}^{*}\left(\pi_{0}\right) \equiv \bar{V}_{E}\left(\pi_{E}^{*} \mid \pi_{0}\right)=\left\{\begin{array}{cl}
\left(\frac{K}{\beta_{1}-1}\right)^{1-\beta_{1}}\left(\frac{\rho_{1} \pi_{0}}{\beta_{1}}\right)^{\beta_{1}} & \text { if } \pi_{0} \leq \pi_{E}^{*} \\
\rho_{1} \pi_{0}-K & \text { if } \pi_{0}>\pi_{E}^{*}
\end{array}\right.
$$


The optimal investment threshold and the value of the option to invest conditional upon the market being in a growth phase are very similar to those derived in a GBM-based setup (c.f. Dixit and Pindyck, 1994, pp. 142-143). Similarly, Proposition 1 implies that the firm chooses to invest when the expected net present value of its investment exceeds $\frac{K}{\beta_{1}-1}>0$, the opportunity cost of exercising the option to invest.

A major contribution of this paper is to improve our conceptual understanding regarding the optimality of a "wait-and-see" approach. In particular, unlike traditional real options models based on Ito processes, the firm's problem can be given the following marginal interpretation:

Proposition 2 The optimal investment threshold $\pi_{E}^{*}$ satisfies the following equation:

$$
\pi_{E}^{*}=r K+\lambda_{1}\left[\delta_{2}\left(\rho_{1} \pi_{E}^{*}-K\right)-\left(\rho_{2} \pi_{E}^{*}-K\right)\right]
$$

Proof. See Appendix B.

Proposition 2 states that the firm must equate the marginal cost and the marginal value of waiting to invest. ${ }^{24}$ The left hand side of equation (3) is the profit flow forgone by delaying entry a short period of time, $\pi_{E}^{*} d t$, while the right hand side of (3) quantifies the marginal value of waiting, which consists of two components. The first part, $r K d t$, is the part of the investment cost saved by waiting an infinitesimal unit of time. The second component, $\lambda_{1} d t\left[\delta_{2}\left(\rho_{1} \pi_{E}^{*}-K\right)-\left(\rho_{2} \pi_{E}^{*}-K\right)\right]$, is the marginal option value of waiting and stems from Bernanke's "bad news principle of irreversible investment." ${ }^{25}$ When the process reaches $\pi_{E}^{*}$, waiting to invest allows the firm to avoid making a poor investment in case the process switches to decline immediately. This happens with conditional probability $\lambda_{1} d t$, and would only bring an expected stream of discounted profits of $\rho_{2} \pi_{E}^{*}-K=\underline{\mathcal{E}}\left(\pi_{E}^{*}, 0\right)-K$, at the expense of sacrificing the option to invest, which would yield an expected payoff of $\delta_{2}\left(\rho_{1} \pi_{E}^{*}-K\right)=\delta_{2}\left[\overline{\mathcal{E}}\left(\pi_{E}^{*}, 0\right)-K\right]$ (accounting for the random-length period of time elapsed until the profit flow grows back to the investment threshold, $\left.\pi_{E}^{*}\right)$.

To conclude with this section, it only remains to show that the value of an investment opportunity experiences an upward (downward) jump when a decline phase stops (starts). This is a relevant result that gives a rationale for using jump processes in the valuation of stock even if the underlying state variable follows a continuous stochastic process. Note that if the firm has not invested, whenever the market switches from growth to decline, the

\footnotetext{
${ }^{24}$ It is worth pointing out that the proposition is unchanged if $p_{0}<1$.

${ }^{25}$ Such principle states that irreversibility yields no advantages but implies some costs because the firm cannot recoup its investment if market conditions turn out to be adverse, which creates the asymmetry that the firm cares only about adverse events — which would not be regrettable were investment reversible - but not favorable ones.
} 
value of an inactive firm switches from $\bar{V}_{E}^{*}\left(\pi_{0}\right)$ to $\underline{V}_{E}^{*}\left(\pi_{0}\right)$, where $\underline{V}_{E}^{*}\left(\pi_{0}\right)$ denotes the firm's value when the market is declining at $\pi_{0}$. Because the firm never invests while the market is declining, it follows from Corollary 1 that $\underline{V}_{E}^{*}\left(\pi_{0}\right)=\delta_{2} \bar{V}_{E}^{*}\left(\pi_{0}\right)<\bar{V}_{E}^{*}\left(\pi_{0}\right) \cdot{ }^{26}$ Although the instantaneous profit stays (roughly) at the same level, the firm's value jumps down (up) significantly whenever the market suddenly stops growing (declining). ${ }^{27}$ This result is formally stated as follows:

Proposition 3 Let $\pi_{0}$ be the current profit flow level. If the firm is inactive, then $\bar{V}_{E}^{*}\left(\pi_{0}\right)>$ $\underline{V}_{E}^{*}\left(\pi_{0}\right)$.

\section{$5 \quad$ Exit decision under uncertainty}

In this section, the firm is assumed to be already active in the market. Although in principle it can operate in the market forever, its (indivisible) asset has a one-time opportunity cost of $S>0$. Moreover, the firm cannot reenter in the future if it exits, i.e., $K=\infty$.

Our main purpose is to analyze and characterize disinvestment behavior in markets that follow a random number of random-length cycles whenever the firm is always unsure about whether the current cycle is the last one. However, the case in which there always exists a positive prior probability that any decline phase will not be the last one is analytically intractable (unless the probability $p_{0}$ is equal to 1 ). ${ }^{28}$ Notwithstanding, one can get around

\footnotetext{
${ }^{26}$ If $p_{0}<1$, then it can be shown that $\underline{V}_{E}^{*}\left(\pi_{0} \mid \bar{\pi}\right)=p\left(\pi_{0} \mid \bar{\pi}\right) \delta_{2} \bar{V}_{E}^{*}\left(\pi_{0}\right) / p_{0}$ (see Theorem 2), where $\underline{V}_{E}^{*}\left(\pi_{0} \mid \bar{\pi}\right)$ denotes the value of the investment opportunity if the market is currently at state $\pi_{0}$ given that it has started declining at state $\bar{\pi} \geq \pi_{0}$. Hence, jumps in firm value also take place in this case, since $p\left(\pi_{0} \mid \bar{\pi}\right) \delta_{2} / p_{0}<1$.

${ }^{27}$ It is worth remarking that this result holds even if the firm becomes active, since $\rho_{1}>\rho_{2}\left(\pi_{0}, \pi_{0}\right) \geq$ $\rho_{2}\left(\pi_{0}, \bar{\pi}\right)$.

${ }^{28}$ Essentially because it requires solving an unwieldy system of two non-linear partial differential equations. To see this, let $\bar{V}^{*}(\cdot)$ and $\underline{V}^{*}(\cdot)$ respectively denote the value of the disinvestment opportunity when the market is growing and declining. Using dynamic programming, we have that the value function if the market started declining at $\bar{\pi}$ and is currently at state $\pi_{0}$ is

$$
\underline{V}^{*}\left(\pi_{0}, \bar{\pi}\right)=\max \left\{S, \pi_{0} d t+(1-r d t)\left[p\left(\pi_{0} \mid \bar{\pi}\right) \lambda_{2} d t \bar{V}^{*}\left(\pi_{0}+d \pi\right)+\left(1-p\left(\pi_{0} \mid \bar{\pi}\right) \lambda_{2} d t\right) \underline{V}^{*}\left(\pi_{0}+d \pi, \bar{\pi}\right)\right]\right\},
$$
}

whereas the value function when the market is growing at state $\pi_{0}$ is

$$
\bar{V}^{*}\left(\pi_{0}\right)=\max \left\{S, \pi_{0} d t+(1-r d t)\left[\left(1-\lambda_{1} d t\right) \bar{V}^{*}\left(\pi_{0}+d \pi\right)+\lambda_{1} d t \underline{V}^{*}\left(\pi_{0}+d \pi, \pi_{0}+d \pi\right)\right]\right\} .
$$

Standard manipulations of this pair of Bellman equations in their waiting regions lead to the following system of non-linear differential equations:

$$
\left(r+\lambda_{1}\right) \bar{V}^{*}\left(\pi_{0}\right)=\pi_{0}+\alpha_{1} \pi_{0} \frac{d \bar{V}^{*}\left(\pi_{0}\right)}{d \pi}+\lambda_{1} \underline{V}^{*}\left(\pi_{0}, \pi_{0}\right)
$$

and

$$
\left(r+\lambda_{2} p\left(\pi_{0} \mid \bar{\pi}\right)\right) \underline{V}^{*}\left(\pi_{0}, \bar{\pi}\right)=\pi_{0}+\lambda_{2} p\left(\pi_{0} \mid \bar{\pi}\right) \bar{V}^{*}\left(\pi_{0}\right)+\alpha_{2} \pi_{0} \frac{\partial \underline{V}^{*}\left(\pi_{0}, \bar{\pi}\right)}{\partial \pi_{0}} .
$$


this drawback and draw several conclusions about the impact on disinvestment of a random number of random-length cycles whenever the probability that a pre-specified cycle will be the last one is equal to 1 . That is, if the decline phase of cycle $N$ (where $N$ is a known integer greater than 1 ) is the last one with probability 1 - if it is ever reached-, but any previous decline phase is the last one only with prior probability $1-p_{0} \in(0,1)$, then it is possible to work backwards from the last cycle and show existence of a unique optimal exit policy function for each cycle. In addition, it is also possible to characterize the properties of such set of functions. The main concern with this approach, of course, has to do with the distortionary effect of the existence of a known last cycle, but such effect vanishes for large enough $N$, and hence has a negligible effect on the exit policy functions for each of the cycles.

The properties of optimal disinvestment behavior whenever a market can go through a maximum of $N \geq 2$ cycles as described above turn out to be quite simple and intuitive. ${ }^{29}$ First, exit never takes place during a period of market growth (except of course at the first growing phase if the profit level is low enough, which is ruled out to make the analysis non-trivial).

Second, decline phases of any cycle that is not the $N$ th one are characterized by an update of the firm's beliefs about the probability that the current downturn will be permanent (in the spirit of Lemma 1). In particular, as no growth phase arrives, the firm becomes more pessimistic about the probability that the market ends up declining forever. Given state $\pi_{0}$, how pessimistic the firm is depends on the level $\bar{\pi}$ at which the market starts its decline, so the firm's optimal disinvestment threshold is a function of $\bar{\pi}$, as well as of the number of the cycle through which the market may go. ${ }^{30}$ For a fixed cycle that is not the $N$ th one, cycle $n \in\{1, \ldots, N-1\}$ say, the optimal exit policy during the decline phase is driven by Bernanke's (1983) "bad news principle." In particular, if immediate exit when the market starts its decline at state $\bar{\pi}$ is not optimal, then the firm must equate the marginal cost of waiting to disinvest and the marginal value of delaying exit. Let us denote the optimal level at which the firm prefers exiting by $\underline{\pi}_{n}^{*}(\bar{\pi})$ if the market started its decline phase at $\bar{\pi}$. Then the firm forgoes $r S d t$ over a short time interval, but gains a flow of profits equal to $\underline{\pi}_{n}^{*} d t$. Yet, deferring exit is valuable as well because there is a marginal option value of waiting to disinvest, which arises due to the possible arrival of bad news. Thus, if the firm

\footnotetext{
${ }^{29}$ The formal arguments that give rise to the explanations that follow can be found in the proof of Proposition 4 below.

${ }^{30}$ Of course, the optimal investment threshold in cycle $N$ does not depend on the starting level and rather is a constant. However, if $N$ is large enough the probability of reaching such a cycle becomes negligible, and we do not have to care about such "pathological" disinvestment behavior.
} 
delays disinvestment for a short time interval, then the firm believes that a growth phase might start with some probability, ${ }^{31}$ and the firm would avoid making a poor exit decision, since not exiting when an upturn arrives can be shown to yield an asset value greater than $S$. Based on the firm's updated information, the probability attributed to the arrival of a growth phase over a short time interval given that it has not yet arrived is $p\left(\underline{\pi}_{n}^{*} \mid \bar{\pi}\right) \lambda_{2} d t$.

Assuming that exit is not optimal at the initial date, and letting $\bar{V}_{n}^{*}\left(\pi_{0}\right)$ denote the value of the disinvestment opportunity if the market is at state $\pi_{0}$ in the growing phase of cycle $n \in\{1, \ldots, N\}$, we can formalize the previous arguments as follows:

Proposition 4 There exists a unique optimal disinvestment policy, and it is such that the firm exits only if the market is declining. In addition, if the market is in the declining phase of cycle $n \in\{1, . ., N-1\}$ at state $\pi_{0}$ and the decline has started at state $\bar{\pi} \geq \pi_{0}$, then there exists a unique $\widehat{\pi}_{n}<r S$ such that the threshold $\underline{\pi}_{n}^{*}(\bar{\pi})$ below which exit is optimal satisfies $\underline{\pi}_{n}^{*}(\bar{\pi})=\bar{\pi}$ for $\bar{\pi} \leq \widehat{\pi}_{n}$ and

$$
r S=\underline{\pi}_{n}^{*}(\bar{\pi})+p\left(\underline{\pi}_{n}^{*}(\bar{\pi}) \mid \bar{\pi}\right) \lambda_{2}\left[\bar{V}_{n+1}^{*}\left(\underline{\pi}_{n}^{*}(\bar{\pi})\right)-S\right]
$$

for $\bar{\pi}>\widehat{\pi}_{n}$. It holds that $\bar{V}_{n+1}^{*}\left(\underline{\pi}_{n}^{*}(\bar{\pi})\right)>S$ for all $\bar{\pi}>\widehat{\pi}_{n}$ and that $\underline{\pi}_{n}^{*}(\bar{\pi})$ is an increasing function that approaches $r S$ as $\bar{\pi} \rightarrow \infty$. As for the optimal exit threshold in the declining phase of cycle $N$, it is equal to $\underline{\pi}_{N}^{*}=\max (r S, \bar{\pi})$.

Proof. See Appendix B.

A few comments about this set of results are in order. First, $\widehat{\pi}_{n}$ is the highest state below which the firm prefers exiting immediately if the market suddenly switches from growth to decline in cycle $n \in\{1, \ldots, N-1\}$ (if such cycle is ever reached, of course). Second, for states $\bar{\pi}>\widehat{\pi}_{n}$ at which the market starts declining, the firm does not find it optimal to disinvest right away after the switching date, and it waits to exit so as to gather information and shield against making a poor disinvestment decision if the market switches back to growth. As can be seen from expression (4), optimal decision-making balances the interest lost on the asset's outside value against the flow of profits that can be harvested by remaining a little bit longer in the market and the marginal change in the option value of waiting to disinvest. This marginal option value stems from the bad news principle of irreversible investment, as we already explained. Furthermore, when computing the marginal option value of delaying exit, the firm takes into account what it learns about the probability of the market experiencing another growth phase based on the information gathered as time goes by. Proposition 4 shows that the higher the state at which the market starts its decline, the

\footnotetext{
${ }^{31}$ Note that the beginning of a growth phase constitutes bad news for an exit decision in a declining market.
} 
higher the threshold that optimally triggers exit, which is quite natural given that the firm starts becoming pessimistic earlier. Third and last, the proof of Proposition 4 uses induction and provides the algorithm for computing the optimal exit policy and the value function for arbitrary $N \geq 2$ (no matter how large $N$ is).

As an illustration of Proposition 4, suppose that $N=3, S=1, r=0.1, \alpha_{1}=0.05=-\alpha_{2}$, $\lambda_{1}=\lambda_{2}=0.1$ and $p_{0}=0.8$. Then it holds that $\underline{\pi}_{3}^{*}(\bar{\pi})=\min (0.1, \bar{\pi})$ (where $\bar{\pi}$ is the state at which the market starts declining in the last cycle). In addition, we have that $\underline{\pi}_{2}^{*}(\bar{\pi})=\bar{\pi}$ if $\bar{\pi} \leq 0.0894$, whereas for $\bar{\pi}>0.0894, \underline{\pi}_{2}^{*}(\bar{\pi})$ is given by the following implicit function:

$$
0.1=\underline{\pi}_{2}^{*}+\frac{0.08\left(\bar{V}_{3}^{*}\left(\underline{\pi}_{2}^{*}\right)-1\right)}{0.8+0.2\left(\bar{\pi} / \underline{\pi}_{2}^{*}\right)^{2}}
$$

where $\bar{V}_{3}^{*}\left(\underline{\pi}_{2}^{*}\right)=\frac{\underline{\pi}_{2}^{*}}{0.15}+0.5+555.556\left(\underline{\pi}_{2}^{*}\right)^{4}$. In turn, $\underline{\pi}_{1}^{*}(\bar{\pi})=\bar{\pi}$ if $\bar{\pi} \leq 0.0881$; else, $\underline{\pi}_{1}^{*}(\bar{\pi})$ is the (unique) solution to the following equation: $0.1=\underline{\pi}_{1}^{*}+\frac{0.08\left(\bar{V}_{2}^{*}\left(\underline{\pi}_{1}^{*}\right)-1\right)}{0.8+0.2\left(\bar{\pi} / \underline{\pi}_{1}^{*}\right)^{2}}$, where $\bar{V}_{2}^{*}(\cdot)$ is defined in the proof of Proposition $4 .{ }^{32}$ Figure 5 plots the three exit thresholds as a function of the state at which the market starts declining (the solid curve represents $\underline{\pi}_{1}^{*}(\cdot)$, whereas the dashed and dotted curves represent $\underline{\pi}_{2}^{*}(\cdot)$ and $\underline{\pi}_{3}^{*}(\cdot)$, respectively). As shown in the proposition, $\underline{\pi}_{1}^{*}(\bar{\pi})$ and $\underline{\pi}_{2}^{*}(\bar{\pi})$ are increasing and converge to 0.1 as $\bar{\pi}$ grows large. Also, $\underline{\pi}_{1}^{*}(\bar{\pi})<\underline{\pi}_{2}^{*}(\bar{\pi})$ for $\bar{\pi}>0.0881$, since the firm finds the outside option more tempting when fewer cycles are left (i.e., the firm becomes less patient).

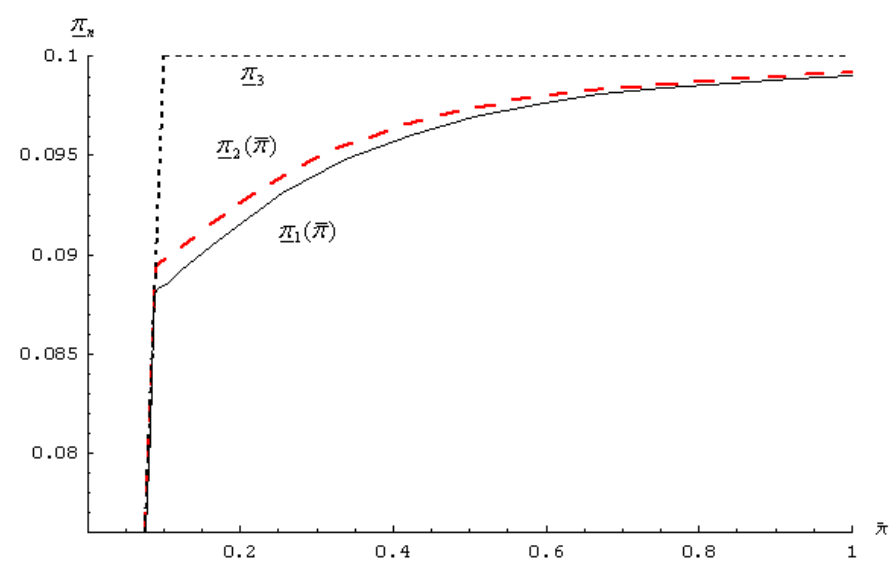

Figure 5: Optimal exit thresholds as a function of $\bar{\pi}$ when $N=3, S=1, r=0.1, \alpha_{1}=0.05=-\alpha_{2}$, $\lambda_{1}=\lambda_{2}=0.1$ and $p_{0}=0.8$

\footnotetext{
${ }^{32}$ We omit the expression for $\bar{V}_{2}^{*}(\cdot)$ because it requires including that for $\underline{V}_{2}^{*}(\cdot)$ because of the recursive structure of the problem.
} 
We now plot a sample path of the process and analyze how the value of the disinvestment opportunity (denoted by $V(t)$ ) evolves based on that sample path. As can be seen in Figure 6 , the switching dates in the sample path (see top of the figure) are $T_{1}=11.20, T_{2}=16.48$, $T_{3}=22.15, T_{4}=47.06$ and $T_{5}=56.90$ because the realized number of cycles is 3 . Note that when the market is going through its first cycle, we have that the declining phase starts at $0.1 e^{0.05(11.20)}=0.1750$, so the firm should have exited at state $\underline{\pi}_{1}^{*}(0.1750)=0.091$ if it had been reached. However, the process starts growing again at state $0.1750 e^{-0.05(16.48-11.20)}=$ 0.1344, so the firm prefers waiting at least until the next declining phase. When the process starts declining again at state $0.1344 e^{0.05(22.15-16.48)}=0.1784$, the firm finds it optimal to wait to exit until state $\underline{\pi}_{2}^{*}(0.1784)=0.092$. Because the sample path reaches such state in the declining phase of the second cycle, the firm exits at $\underline{\pi}_{2}^{*}(0.1784)$, that is, at time $T_{X}=35.40$. The bottom of the figure shows the sample path of the value of the disinvestment opportunity, which is characterized by upward (downward) jumps whenever a growth phase begins (stops), unless the firm chooses to remain inactive.

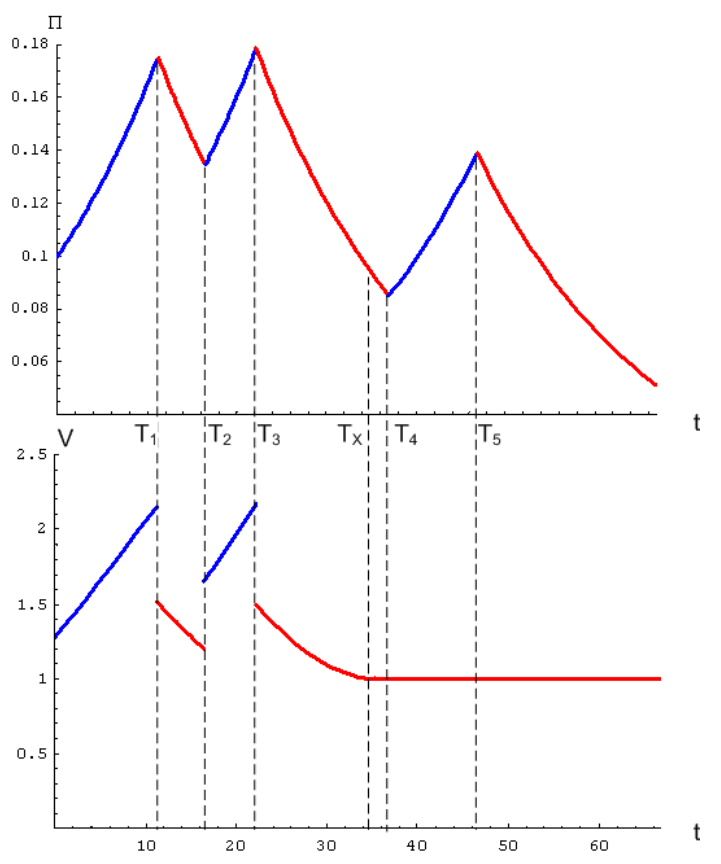

Figure 6: Sample path of $\Pi(t)$ and induced sample path of $V(t)$ when $N=3, S=1, r=0.1$, $\alpha_{1}=0.05=-\alpha_{2}, \lambda_{1}=\lambda_{2}=0.1$ and $p_{0}=0.8$

Despite it can be seen from this numerical example that the value of the disinvestment opportunity experiences jumps upwards (downwards) whenever an upturn (downturn) starts, it has proven intractable when trying to show it analytically. However, this conclusion (and several others) can be easily drawn with the aid of the mathematical results in Section 3 in 
the relevant case in which $p_{0}=1$ (and $N=\infty$ ). In this case, it can be shown that exit takes place only if the market is declining, ${ }^{33}$ so the homogeneity of the (Markov) stochastic process implies that the firm simply chooses a threshold $\pi_{X}$ such that the firm exits the market the first time the process hits such threshold from above. Formally, the firm solves the following problem:

$$
\begin{aligned}
\max _{\pi_{X}} \underline{V}_{X}\left(\pi_{X} \mid \pi_{0}\right) & =\underline{\mathcal{E}}\left(\pi_{0}, \pi_{X} \mid \pi_{0}\right)+S \underline{\varphi}_{2}\left(\pi_{0}, \pi_{X} \mid \pi_{0}\right) \\
& =\rho_{2}\left(\pi_{0}, \pi_{0}\right) \pi_{0}\left[1-\left(\frac{\pi_{0}}{\pi_{X}}\right)^{\beta_{2}-1}\right]+S\left(\frac{\pi_{0}}{\pi_{X}}\right)^{\beta_{2}} .
\end{aligned}
$$

Thus, starting from a level $\pi_{0}$ at which the market is declining, the firm collects a discounted stream of profits until state $\pi_{X}<\pi_{0}$ is first reached (from above). The value of such discounted profit stream while transitioning from $\pi_{0}$ to $\pi_{X}$ is $\underline{\mathcal{E}}\left(\pi_{0}, \pi_{X} \mid \pi_{0}\right)=$ $\rho_{2}\left(\pi_{0}, \pi_{0}\right) \pi_{0}\left[1-\left(\pi_{0} / \pi_{X}\right)^{\beta_{2}-1}\right]$, as shown by Theorem 4 for the case in which $p_{0}=1$ (since in such a case $\phi=1)$. However, there is an additional source of value, since the firm seizes the outside option, whose value is $S$, when state $\pi_{X}$ is first reached. The proper expected discount factor, given that the market is in a decline phase, is $\underline{\varphi}_{2}\left(\pi_{0}, \pi_{X} \mid \pi_{0}\right)=\left(\pi_{0} / \pi_{X}\right)^{\beta_{2}}$, as shown by Theorem 3 (for $p_{0}=1$ ).

Since $p_{0}=1$ implies that $\rho_{2}\left(\pi_{0}, \pi_{0}\right)$ does not actually depend on $\pi_{0}$, we can drop its arguments. Noticing that $\underline{V}_{X}\left(\pi_{X} \mid \pi_{0}\right)$ is strictly quasi-concave and

$$
\frac{\partial \underline{V}_{X}\left(\pi_{X} \mid \pi_{0}\right)}{\partial \pi_{X}}=\left[\frac{\rho_{2}\left(\beta_{2}-1\right) \pi_{X}-\beta_{2} S}{\pi_{X}}\right]\left(\frac{\pi_{0}}{\pi_{X}}\right)^{\beta_{2}}
$$

it is easy to prove the following results:

Proposition 5 Suppose that the market is declining and that the firm is currently active. Then the firm's optimal exit rule is to disinvest as soon as the market reaches state

$$
\pi_{X}^{*}=\frac{1}{\rho_{2}} \frac{\beta_{2}}{\beta_{2}-1} S,
$$

\footnotetext{
${ }^{33}$ The argument is identical in spirit to the one used for entry, and it makes use of the fact that $r>$ $\frac{\beta_{2}}{\left(\beta_{2}-1\right) \rho_{2}}$, which is shown in the proof of Proposition 7 .
} 
where $\beta_{2}=\frac{\alpha_{1}\left(r+\lambda_{2}\right)+\alpha_{2}\left(r+\lambda_{1}\right)+\sqrt{\Delta}}{2 \alpha_{1} \alpha_{2}}<0$. The value of the firm is

$$
\underline{V}_{X}^{*}\left(\pi_{0}\right) \equiv \underline{V}_{X}\left(\pi_{X}^{*} \mid \pi_{0}\right)=\left\{\begin{array}{cc}
\rho_{2} \pi_{0}+\left(S-\rho_{2} \pi_{X}^{*}\right)\left(\frac{\pi_{0}}{\pi_{X}^{*}}\right)^{\beta_{2}} & \text { if } \pi_{0} \geq \pi_{X}^{*} \\
S & \text { if } \pi_{0}<\pi_{X}^{*}
\end{array} .\right.
$$

Similarly to the canonical real options literature, Proposition 5 implies that the firm exits when the expected net present value of its disinvestment falls below $S /\left(\beta_{2}-1\right)<0$, since there exists an opportunity cost for exercising the option to disinvest. As with the entry problem, the value of the option to disinvest conditional upon the market being in a decline phase is quite similar to that derived in a Geometric Brownian Motion setting. The same applies to the optimal disinvestment threshold.

As usual, the firm's optimal exit rule lends itself to a marginal interpretation linked to the "bad news principle":

Proposition 6 The optimal disinvestment threshold $\pi_{X}^{*}$ satisfies the following equation:

$$
r S=\pi_{X}^{*}+\lambda_{2}\left(\gamma_{1} \pi_{X}^{*}+\delta_{1} S-S\right)
$$

Proof. See Appendix B.

When deciding whether or not to exit the market, the firm must compare the marginal value and the marginal cost of waiting. By delaying exit a short time period of length $d t$, the firm forgoes earning (approximately) $r S d t$. As usual, the marginal value of waiting to disinvest consists of two components. Delaying exit a little bit allows the firm to reap a flow of profits equal to $\pi_{X}^{*} d t$. There is also a marginal option value of waiting to disinvest, which of course stems from avoiding making a poor disinvestment decision. With conditional probability $\lambda_{2} d t$, the market switches to growth, so waiting would allow the firm to remain in operation and keep the option to exit in the future alive, which is worth $\gamma_{1} \pi_{X}^{*}+\delta_{1} S$, at the expense of sacrificing $S .{ }^{34}$

To conclude this section, we show that the value of a disinvestment opportunity experiences an upward (downward) jump if a declining phase ends (begins). Our next proposition establishes this result, which is conceptually very different from Proposition 1. Thus, while the jump in the firm's value for a potential entrant is simply due to the discount factor re-

\footnotetext{
${ }^{34}$ Note from Corollary 2 that $\gamma_{1} \pi_{X}^{*}$ quantifies the expected stream of discounted profits while the market transitions from a state $\pi_{X}^{*}$ in which the market is growing until such state is first hit from above. In turn, note from Corollary 1 that $\delta_{1} S$ quantifies the expected discounted value of getting an asset worth $S$ when the market first reaches state $\pi_{X}^{*}$ from above, given that the market is currently at a growing phase whose state is $\pi_{X}^{*}$.
} 
lated to the delay in entry caused by a regime shift, the abrupt change of value for an active firm is the result of a trade-off between the expected stream of discounted profits reaped and the delayed recovery of the outside value. More specifically, if the market is declining at $\pi_{0}>\pi_{X}^{*}$, the firm has a value equal to $\underline{V}_{X}^{*}\left(\pi_{0}\right)$. However, if the market switches to growth at $\pi_{0}$, then the firm's value function becomes $\bar{V}_{X}^{*}\left(\pi_{0}\right)=\gamma_{1} \pi_{0}+\delta_{1} \underline{V}_{X}^{*}\left(\pi_{0}\right)$, because the firm never exits a growing market and hence must wait at least until the profit flow comes back to the same state. The first term, $\gamma_{1} \pi_{0}$, denotes the expected stream of discounted profits collected while the market moves from state $\pi_{0}$ until it first comes back to $\pi_{0}$ from above (see Corollary 2). The second term, $\delta_{1} \underline{V}_{X}^{*}\left(\pi_{0}\right)$, denotes the expected continuation value after the market declines back to state $\pi_{0}$ (see Corollary 1 ). By comparing $\underline{V}_{X}^{*}\left(\pi_{0}\right)$ and $\bar{V}_{X}^{*}\left(\pi_{0}\right)$, we have the following result:

Proposition 7 Let $\pi_{0}$ be the current state of the market. If the firm is active, then $\bar{V}_{X}^{*}\left(\pi_{0}\right)>$ $\underline{V}_{X}^{*}\left(\pi_{0}\right)$.

Proof. See Appendix B.

\section{Conclusion}

This paper has modeled the stochastic evolution of demand for high-tech goods by means of a regime-switching stochastic process in which the regime shifts a random number of times. We have studied a firm's optimal (dis)investment behavior in this type of markets under the assumption that the realized number of regime switches is never observed. This creates a signal extraction problem because the firm can update its beliefs about the probability that the regime does not experience any additional shifts based on the length of the period during which the regime does not switch. Given this setting in which flow profit alternates between growth and decline phases at random times, we have shown that the existence of regime shifts gives rise to an option value of waiting to (dis)invest and to discontinuities in the sample path of firm value. Thus, even though the sample path of profits is continuous, firm value experiences jumps upwards (downwards) whenever a growth (decline) phase starts. This holds regardless of whether the firm has an option to invest or an option to disinvest. Lastly, we have provided a marginal interpretation to optimal entry/exit rules in the light of the bad news principle of irreversible investment, thus providing a strong conceptual foundation to the theory of real options.

There are at least a couple of aspects that are worthwhile emphasizing about our framework and that may prove to be useful for future work on the properties of irreversible 
(dis)investment in stochastically cyclical markets. On the one hand, we would like to remark that our setting does not require making use of the heavy mathematical apparatus traditionally employed by the real options literature. For instance, direct applications of our theorems when there is no signal extraction problem allow us to avoid using stochastic calculus and even dynamic programming techniques when solving (dis)investment models that involve lumpiness. As we have shown, (dis)investment timing problems can be directly formulated and solved using ordinary calculus and are amenable to an economic interpretation. Indeed, if reentry is not allowed, it is straightforward to solve analytically the problem faced by a single firm that must choose when to enter and when to exit. The case in which there is a signal extraction problem is typically more complex from an analytical standpoint, and may require using numerical methods when solving it (e.g., in the case of exit). Still, we believe this is an important feature of the dynamics of product markets such as those for high-tech goods. Because such markets are usually oligopolistic, it would be interesting to analyze the dynamics of competition among several firms with private information about the prior probability that the current cycle is the last one. For the case of disinvestment, we conjecture that firms would play a war of attrition in which the winner would face a "survivor's curse" (see Moscarini and Squintani 2004).

Another aspect worthwhile pointing out is that the model can be enriched in order to improve its explanatory power, possibly at the expense of analytical tractability. Thus, when a switching date is realized, the sample path of the process that governs profit evolution is assumed to change to a different but known growth rate. Assuming that the growth rate is random may lead to possibly different (dis)investment dynamics given a market evolution that need not be stochastically cyclical despite the regime shifts. This may prove fruitful for the construction of random processes that better represent the stochastic dynamics of a variety of economic and financial variables. Both theoretical and empirical work may benefit from pursuing this promising research avenue. 


\section{Appendix A}

Proof of Lemma 1. By Bayes' rule, the posterior probability that the current decline phase is not the last one given that the period of time elapsed between $\bar{\pi}$ and $\pi_{0}$ is $t\left(\pi_{0}, \bar{\pi}\right)=$ $\frac{1}{\alpha_{2}} \ln \left(\pi_{0} / \bar{\pi}\right)$ equals $p\left(\pi_{0} \mid \bar{\pi}\right)=\frac{e^{-\lambda_{2} t\left(\pi_{0}, \bar{\pi}\right)} p_{0}}{e^{-\lambda_{2} t\left(\pi_{0}, \bar{\pi}\right)} p_{0}+1-p_{0}}$, so it is straightforward to see that $p\left(\pi_{0} \mid \bar{\pi}\right)=\frac{p_{0}\left(\bar{\pi} / \pi_{0}\right)^{\frac{\lambda_{2}}{\alpha_{2}}}}{p_{0}\left(\bar{\pi} / \pi_{0}\right)^{\frac{\lambda_{2}}{\alpha_{2}}}+\left(1-p_{0}\right)}$. That the posterior belief increases with $\pi_{0}$ can be seen by inspection.

Proof of Theorem 1. Let $\overline{\mathcal{E}}\left(\pi_{0}, 0\right)$ denote the expected stream of discounted profits when the process is growing at $\pi_{0}$. Also, let $\underline{\mathcal{E}}\left(\pi_{0}, 0 \mid \bar{\pi}\right)$ denote the expected stream of discounted profits when the process started declining at $\bar{\pi}$ and the current level of the process is $\pi_{0}$ (the expectation is conditional upon the information gathered during the transition between states $\bar{\pi}$ and $\left.\pi_{0}\right)$. We can relate $\overline{\mathcal{E}}\left(\pi_{0}, 0\right)$ and $\underline{\mathcal{E}}\left(\pi_{0}, 0 \mid \bar{\pi}\right)$ as follows:

$$
\overline{\mathcal{E}}\left(\pi_{0}, 0\right)=\int_{0}^{\infty} \lambda_{1} e^{-\lambda_{1} \tau_{1}}\left[\int_{0}^{\tau_{1}} \pi_{0} e^{\alpha_{1} s} e^{-r s} d s+\underline{\mathcal{E}}\left(\pi_{0} e^{\alpha_{1} \tau_{1}}, 0 \mid \pi_{0} e^{\alpha_{1} \tau_{1}}\right) e^{-r \tau_{1}}\right] d \tau_{1}
$$

and

$$
\begin{aligned}
\underline{\mathcal{E}}\left(\pi_{0}, 0 \mid \bar{\pi}\right)= & \left(1-p\left(\pi_{0} \mid \bar{\pi}\right)\right) \int_{0}^{\infty} \pi_{0} e^{\alpha_{2} s} e^{-r s} d s+ \\
& p\left(\pi_{0} \mid \bar{\pi}\right) \int_{0}^{\infty} \lambda_{2} e^{-\lambda_{2} \tau_{2}}\left[\int_{0}^{\tau_{2}} \pi_{0} e^{\alpha_{2} s} e^{-r s} d s+\overline{\mathcal{E}}\left(\pi_{0} e^{\alpha_{2} \tau_{2}}, 0\right) e^{-r \tau_{2}}\right] d \tau_{2} .
\end{aligned}
$$

To see how $\overline{\mathcal{E}}\left(\pi_{0}, 0\right)$ arises, note that the process will start declining at some random future date $\tau_{1}$, so the firm gets a stream of discounted profits until the switching date, together with an asset worth $\underline{\mathcal{E}}\left(\pi_{0} e^{\alpha_{1} \tau_{1}}, 0 \mid \pi_{0} e^{\alpha_{1} \tau_{1}}\right) e^{-r \tau_{1}}$. Regarding $\underline{\mathcal{E}}\left(\pi_{0}, 0 \mid \bar{\pi}\right)$, note that with updated probability $1-p\left(\pi_{0} \mid \bar{\pi}\right)$ the process will never grow again, and hence the firm collects a discounted profit stream given that the process declines forever. With probability $p\left(\pi_{0} \mid \bar{\pi}\right)$, though, the process will expand at some random future date $\tau_{2}$, so the firm collects a discounted profit stream until the process stops declining, and the discounted value of an asset worth $\overline{\mathcal{E}}\left(\pi_{0} e^{\alpha_{2} \tau_{2}}, 0\right)$ at such switching date.

Because $p\left(\pi_{0} \mid \pi_{0}\right)=p_{0}$ and $\overline{\mathcal{E}}\left(\pi_{0} e^{\alpha_{2} \tau_{2}}, 0\right)=e^{\alpha_{2} \tau_{2}} \overline{\mathcal{E}}\left(\pi_{0}, 0\right)$ (since random variables are independent), some simple manipulations in (8) imply that

$$
\underline{\mathcal{E}}\left(\pi_{0}, 0 \mid \pi_{0}\right)=\frac{\left[r-\alpha_{2}+\left(1-p_{0}\right) \lambda_{2}\right] \pi_{0}}{\left(r-\alpha_{2}\right)\left(r+\lambda_{2}-\alpha_{2}\right)}+\frac{p_{0} \lambda_{2} \overline{\mathcal{E}}\left(\pi_{0}, 0\right)}{r+\lambda_{2}-\alpha_{2}} .
$$


As a result, it follows that

$$
\underline{\mathcal{E}}\left(\pi_{0} e^{\alpha_{1} \tau_{1}}, 0 \mid \pi_{0} e^{\alpha_{1} \tau_{1}}\right)=\frac{\left[r-\alpha_{2}+\left(1-p_{0}\right) \lambda_{2}\right] \pi_{0} e^{\alpha_{1} \tau_{1}}}{\left(r-\alpha_{2}\right)\left(r+\lambda_{2}-\alpha_{2}\right)}+\frac{p_{0} \lambda_{2} \overline{\mathcal{E}}\left(\pi_{0} e^{\alpha_{1} \tau_{1}}, 0\right)}{r+\lambda_{2}-\alpha_{2}},
$$

whence (7) yields

$$
\overline{\mathcal{E}}\left(\pi_{0}, 0\right)=\left[\frac{\left(r-\alpha_{2}\right)\left(r+\lambda_{1}+\lambda_{2}-\alpha_{2}\right)+\lambda_{1} \lambda_{2}\left(1-p_{0}\right)}{\left(r-\alpha_{2}\right)\left[\left(r+\lambda_{2}-\alpha_{2}\right)\left(r+\lambda_{1}-\alpha_{1}\right)-p_{0} \lambda_{2} \lambda_{1}\right]}\right] \pi_{0}
$$

Hence, $\overline{\mathcal{E}}\left(\pi_{0}, 0\right)=\rho_{1} \pi_{0}$, as we claimed.

As for the functional form of $\underline{\mathcal{E}}\left(\pi_{0}, 0 \mid \bar{\pi}\right)$, performing some manipulations in (8) using that $\overline{\mathcal{E}}\left(\pi_{0} e^{\alpha_{2} \tau_{2}}, 0\right)=\rho_{1} \pi_{0} e^{\alpha_{2} \tau_{2}}$ and that

$$
\frac{\left[\left(r-\alpha_{2}\right) \rho_{1}-1\right] \lambda_{2}}{\left(r+\lambda_{2}-\alpha_{2}\right)}=\frac{\lambda_{2}\left(\alpha_{1}-\alpha_{2}\right)}{\left[\left(r+\lambda_{2}-\alpha_{2}\right)\left(r+\lambda_{1}-\alpha_{1}\right)-p_{0} \lambda_{2} \lambda_{1}\right]}
$$

lead to

$$
\begin{aligned}
\underline{\mathcal{E}}\left(\pi_{0}, 0 \mid \bar{\pi}\right) & =\left[\frac{1}{r-\alpha_{2}}\left(1+\frac{\left[\left(r-\alpha_{2}\right) \rho_{1}-1\right] \lambda_{2} p\left(\pi_{0} \mid \bar{\pi}\right)}{\left(r+\lambda_{2}-\alpha_{2}\right)}\right)\right] \pi_{0} \\
& =\rho_{2}\left(\pi_{0}, \bar{\pi}\right) \pi_{0} .
\end{aligned}
$$

To complete the proof, notice that the inequality in (1) holds by assumption and just make use of the fact that $0<\rho_{2}\left(\pi_{0}, \bar{\pi}\right) \leq \rho_{2}\left(\pi_{0}, \pi_{0}\right)<\rho_{1}$.

Proof of Theorem 2. Let $\bar{\varphi}_{1}\left(\pi_{0}, \pi^{*}\right)$ denote the expected discounted value of a claim to a dollar when the process first hits $\pi^{*}$ from below, conditional on the process being in a growth phase and on its state being $\pi_{0}<\pi^{*}$. Also, let $\underline{\varphi}_{1}\left(\pi_{0}, \pi^{*} \mid \bar{\pi}\right)$ denote the expected discounted value of a claim to a dollar when the process first hits $\pi^{*}$ from below, conditional on the process being in a decline phase that started at level $\bar{\pi}$ and on the current state being $\pi_{0} \leq \bar{\pi}$. By definition, the following holds given our (memoryless) assumptions on the independent random variables involved:

$$
\begin{aligned}
\bar{\varphi}_{1}\left(\pi_{0}, \pi^{*}\right)= & \int_{0}^{\frac{1}{\alpha_{1}} \ln \left(\pi^{*} / \pi_{0}\right)} \lambda_{1} e^{-\lambda_{1} \tau_{1}} \underline{\varphi}_{1}\left(\pi_{0} e^{\alpha_{1} \tau_{1}}, \pi^{*} \mid \pi_{0} e^{\alpha_{1} \tau_{1}}\right) e^{-r \tau_{1}} d \tau_{1}+ \\
& \int_{\frac{1}{\alpha_{1}} \ln \left(\pi^{*} / \pi_{0}\right)}^{\infty} \lambda_{1} e^{-\lambda_{1} \tau_{1}} e^{-r \frac{1}{\alpha_{1}} \ln \left(\pi^{*} / \pi_{0}\right)} d \tau_{1}
\end{aligned}
$$

and

$$
\underline{\varphi}_{1}\left(\pi_{0}, \pi^{*} \mid \bar{\pi}\right)=p\left(\pi_{0} \mid \bar{\pi}\right) \int_{0}^{\infty} \lambda_{2} e^{-\lambda_{2} \tau_{2}} \bar{\varphi}_{1}\left(\pi_{0} e^{\alpha_{2} \tau_{2}}, \pi^{*}\right) e^{-r \tau_{2}} d \tau_{2}
$$


To understand the relation between $\bar{\varphi}_{1}\left(\pi_{0}, \pi^{*}\right)$ and $\underline{\varphi}_{1}\left(\pi_{0}, \pi^{*} \mid \bar{\pi}\right)$, note that if the realized length of the growth phase $\tau_{1}$ is smaller than $\frac{1}{\alpha_{1}} \ln \left(\frac{\pi^{*}}{\pi_{0}}\right)$ (the time it would take to hit state $\pi^{*}$ from below during the current growth phase), then the firm would acquire an asset whose discounted value would be $\underline{\varphi}_{1}\left(\pi_{0} e^{\alpha_{1} \tau_{1}}, \pi^{*} \mid \pi_{0} e^{\alpha_{1} \tau_{1}}\right) e^{-r \tau_{1}}$; if $\tau_{1}$ were greater than $\frac{1}{\alpha_{1}} \ln \left(\frac{\pi^{*}}{\pi_{0}}\right)$, then the firm would acquire the discounted value of a dollar. As for $\underline{\varphi}_{1}\left(\pi_{0}, \pi^{*} \mid \bar{\pi}\right)$, note that with updated probability $1-p\left(\pi_{0} \mid \bar{\pi}\right)$ the process will permanently decline and hence it will never hit state $\pi^{*}$ from below, whereas with complementary probability the process will start growing at some random future date $\tau_{2}$, and hence the firm will acquire at such date an asset whose discounted value is $\bar{\varphi}_{1}\left(\pi_{0} e^{\alpha_{2} \tau_{2}}, \pi^{*}\right) e^{-r \tau_{2}}$.

To solve the system of functional equations that consists of (9) and (10), guess that $\bar{\varphi}_{1}\left(\pi_{0}, \pi^{*}\right)=Y\left(\frac{\pi_{0}}{\pi^{*}}\right)^{\theta_{1}}$ (where $Y$ and $\theta_{1}$ are constants to be found out), and use such functional form for $\bar{\varphi}_{1}\left(\pi_{0}, \pi^{*}\right)$ in (10) so as to get:

$$
\underline{\varphi}_{1}\left(\pi_{0}, \pi^{*} \mid \bar{\pi}\right)=\frac{Y p\left(\pi_{0} \mid \bar{\pi}\right) \lambda_{2}}{r+\lambda_{2}-\alpha_{2} \theta_{1}}\left(\frac{\pi_{0}}{\pi^{*}}\right)^{\theta_{1}} .
$$

Because $p\left(\pi_{0} \mid \pi_{0}\right)=p_{0}$, we have

$$
\underline{\varphi}_{1}\left(\pi_{0} e^{\alpha_{1} \tau_{1}}, \pi^{*} \mid \pi_{0} e^{\alpha_{1} \tau_{1}}\right)=\frac{Y p_{0} \lambda_{2} e^{\alpha_{1} \theta_{1} \tau_{1}}}{r+\lambda_{2}-\alpha_{2} \theta_{1}}\left(\frac{\pi_{0}}{\pi^{*}}\right)^{\theta_{1}}
$$

so (9) becomes:

$$
\begin{aligned}
\bar{\varphi}_{1}\left(\pi_{0}, \pi^{*}\right) & =\frac{Y p_{0} \lambda_{1} \lambda_{2}}{r+\lambda_{2}-\alpha_{2} \theta_{1}}\left(\frac{\pi_{0}}{\pi^{*}}\right)^{\theta_{1}} \int_{0}^{\frac{1}{\alpha_{1}} \ln \left(\pi^{*} / \pi_{0}\right)} e^{-\left(r+\lambda_{1}-\alpha_{1} \theta_{1}\right) \tau_{1}} d \tau_{1}+\left(\frac{\pi_{0}}{\pi^{*}}\right)^{\frac{r+\lambda_{1}}{\alpha_{1}}} \\
& =\frac{Y p_{0} \lambda_{1} \lambda_{2}}{\left(r+\lambda_{2}-\alpha_{2} \theta_{1}\right)\left(r+\lambda_{1}-\alpha_{1} \theta_{1}\right)}\left(\frac{\pi_{0}}{\pi^{*}}\right)^{\theta_{1}}\left[1-\left(\frac{\pi_{0}}{\pi^{*}}\right)^{\frac{r+\lambda_{1}-\alpha_{1} \theta_{1}}{\alpha_{1}}}\right]+\left(\frac{\pi_{0}}{\pi^{*}}\right)^{\frac{r+\lambda_{1}}{\alpha_{1}}}
\end{aligned}
$$

We assumed that $\bar{\varphi}_{1}\left(\pi_{0}, \pi^{*}\right)=Y\left(\frac{\pi_{0}}{\pi^{*}}\right)^{\theta_{1}}$, so the following must hold:

$$
Y\left(\frac{\pi_{0}}{\pi^{*}}\right)^{\theta_{1}}-\left(\frac{\pi_{0}}{\pi^{*}}\right)^{\frac{\lambda_{1}+r}{\alpha_{1}}}=\frac{Y p_{0} \lambda_{1} \lambda_{2}}{\left(r+\lambda_{2}-\alpha_{2} \theta_{1}\right)\left(r+\lambda_{1}-\alpha_{1} \theta_{1}\right)}\left[\left(\frac{\pi_{0}}{\pi^{*}}\right)^{\theta_{1}}-\left(\frac{\pi_{0}}{\pi^{*}}\right)^{\frac{r+\lambda_{1}}{\alpha_{1}}}\right] .
$$

Therefore, $Y=1$, whereas $\theta_{1}$ must satisfy the following equation:

$$
\left(r+\lambda_{2}-\alpha_{2} \theta_{1}\right)\left(r+\lambda_{1}-\alpha_{1} \theta_{1}\right)=p_{0} \lambda_{1} \lambda_{2}
$$

There exist two values of $\theta_{1}$ that solve this quadratic equation. One of the roots, $\beta_{1}$ say, can be easily shown to be greater than 1 (since $\left.\lambda_{1}\left(r+\lambda_{2}\left(1-p_{0}\right)-\alpha_{2}\right)+\left(r+\lambda_{2}-\alpha_{2}\right)\left(r-\alpha_{1}\right)>0\right)$, 
whereas the other one, $\beta_{2}$ say, is negative (since $\left.\left(r+\lambda_{2}-\alpha_{2}\right)\left(r+\lambda_{1}-\alpha_{1}\right)>p_{0} \lambda_{1} \lambda_{2}\right)$. Clearly, only $\beta_{1}$ can be an admissible solution, so recalling that

$$
\Delta=\left[\alpha_{1}\left(\lambda_{2}+r\right)-\alpha_{2}\left(\lambda_{1}+r\right)\right]^{2}+4 p_{0} \alpha_{1} \alpha_{2} \lambda_{1} \lambda_{2}
$$

yields that

$$
\theta_{1}=\beta_{1} \equiv \frac{\alpha_{1}\left(r+\lambda_{2}\right)+\alpha_{2}\left(r+\lambda_{1}\right)-\sqrt{\Delta}}{2 \alpha_{1} \alpha_{2}}
$$

Also, note that expression (11) with $Y=1$ and $\theta_{1}=\beta_{1}$ implies that

$$
\begin{aligned}
\underline{\varphi}_{1}\left(\pi_{0}, \pi^{*} \mid \bar{\pi}\right) & =\frac{p\left(\pi_{0} \mid \bar{\pi}\right) \lambda_{2}}{r+\lambda_{2}-\alpha_{2} \beta_{1}}\left(\frac{\pi_{0}}{\pi^{*}}\right)^{\beta_{1}} \\
& =\left(-\frac{\alpha_{1}\left(r+\lambda_{2}\right)-\alpha_{2}\left(r+\lambda_{1}\right)-\sqrt{\Delta}}{2 \alpha_{2} \lambda_{1}}\right) \frac{p\left(\pi_{0} \mid \bar{\pi}\right)}{p_{0}}\left(\frac{\pi_{0}}{\pi^{*}}\right)^{\beta_{1}}
\end{aligned}
$$

since

$$
r+\lambda_{2}-\alpha_{2} \beta_{1}=\frac{\alpha_{1}\left(r+\lambda_{2}\right)-\alpha_{2}\left(r+\lambda_{1}\right)+\sqrt{\Delta}}{2 \alpha_{1}}=\frac{\left[\alpha_{1}\left(r+\lambda_{2}\right)-\alpha_{2}\left(r+\lambda_{1}\right)\right]^{2}-\Delta}{2 \alpha_{1}\left[\alpha_{1}\left(r+\lambda_{2}\right)-\alpha_{2}\left(r+\lambda_{1}\right)-\sqrt{\Delta}\right]}
$$

and $\left[\alpha_{1}\left(\lambda_{2}+r\right)-\alpha_{2}\left(\lambda_{1}+r\right)\right]^{2}-\Delta=-4 p_{0} \alpha_{1} \alpha_{2} \lambda_{1} \lambda_{2}$. This completes the proof.

Proof of Theorem 3. Let $\bar{\varphi}_{2}\left(\pi_{0}, \pi^{*}\right)$ denote the expected discounted value of a claim to a dollar when the process first hits $\pi^{*}$ from above, conditional on the process being in a growth phase and on the current state being $\pi_{0} \geq \pi^{*}$. In addition, let $\underline{\varphi}_{2}\left(\pi_{0}, \pi^{*} \mid \bar{\pi}\right)$ denote the expected discounted value of a claim to a dollar when the process first hits $\pi^{*}$ from above, conditional on the process being in a decline phase that started at level $\bar{\pi}$ and on the current state being $\pi_{0} \in\left(\pi^{*}, \bar{\pi}\right]$. Then we have the following:

$$
\bar{\varphi}_{2}\left(\pi_{0}, \pi^{*}\right)=\int_{0}^{\infty} \lambda_{1} e^{-\lambda_{1} \tau_{1}} \underline{\varphi}_{2}\left(\pi_{0} e^{\alpha_{1} \tau_{1}}, \pi^{*} \mid \pi_{0} e^{\alpha_{1} \tau_{1}}\right) e^{-r \tau_{1}} d \tau_{1}
$$

and

$$
\begin{aligned}
\underline{\varphi}_{2}\left(\pi_{0}, \pi^{*} \mid \bar{\pi}\right)= & \left(1-p\left(\pi_{0} \mid \bar{\pi}\right)\right) e^{-r \frac{1}{\alpha_{2}} \ln \left(\pi^{*} / \pi_{0}\right)}+ \\
& p\left(\pi_{0} \mid \bar{\pi}\right)\left(\begin{array}{c}
\int_{0}^{\frac{1}{\alpha_{2}} \ln \left(\pi^{*} / \pi_{0}\right)} \lambda_{2} e^{-\lambda_{2} \tau_{2}} \bar{\varphi}_{2}\left(\pi_{0} e^{\alpha_{2} \tau_{2}}, \pi^{*}\right) e^{-r \tau_{2}} d \tau_{2}+ \\
\int_{\frac{1}{\alpha_{2}} \ln \left(\pi^{*} / \pi_{0}\right)}^{\infty} \lambda_{2} e^{-\lambda_{2} \tau_{2}} e^{-r \frac{1}{\alpha_{2}} \ln \left(\pi^{*} / \pi_{0}\right)} d \tau_{2}
\end{array}\right) .
\end{aligned}
$$

To understand the relation between $\bar{\varphi}_{2}\left(\pi_{0}, \pi^{*}\right)$ and $\underline{\varphi}_{2}\left(\pi_{0}, \pi^{*} \mid \bar{\pi}\right)$, note that if the process is going through a growth phase at $\pi_{0}$, then state $\pi^{*} \leq \pi_{0}$ will never be hit in such a phase. 
Hence, when a switching date is realized at $\tau_{1}$, the firm will get $\underline{\varphi}_{2}\left(\pi_{0} e^{\alpha_{1} \tau_{1}}, \pi^{*} \mid \pi_{0} e^{\alpha_{1} \tau_{1}}\right) e^{-r \tau_{1}}$. However, if the process is going through a declining phase at $\pi_{0}$, and such phase has started at $\bar{\pi} \geq \pi_{0}$, then with updated probability $1-p\left(\pi_{0} \mid \bar{\pi}\right)$ the process hits level $\pi^{*}<\pi_{0}$ for sure and hence the firm gets the properly discounted value of a dollar. With complementary probability $p\left(\pi_{0} \mid \bar{\pi}\right)$, the process will grow at some random future date $\tau_{2}$, so two situations must be distinguished. On the one hand, if the process starts declining before reaching $\pi^{*}$ at the random date $\tau_{2}$, then the firm will attain $\bar{\varphi}_{2}\left(\pi_{0} e^{\alpha_{2} \tau_{2}}, \pi^{*}\right) e^{-r \tau_{2}}$. On the other hand, if the declining process goes all the way down to $\pi^{*}$, then the firm will attain the discounted value of the dollar when state $\pi^{*}$ is hit for the first time.

Suppose that $\bar{\varphi}_{2}\left(\pi_{0}, \pi^{*}\right)=A\left(\frac{\pi_{0}}{\pi^{*}}\right)^{\theta_{2}}+C\left(\frac{\pi_{0}}{\pi^{*}}\right)^{\frac{r}{\alpha_{2}}}$ (where $A, C$, and $\theta_{2}$ are constants to be found out). In this case, letting $\bar{p} \equiv p\left(\pi_{0} \mid \bar{\pi}\right)$, we have

$$
\begin{aligned}
& \underline{\varphi}_{2}\left(\pi_{0}, \pi^{*} \mid \bar{\pi}\right)=(1-\bar{p})\left(\frac{\pi_{0}}{\pi^{*}}\right)^{\frac{r}{\alpha_{2}}}+\bar{p}\left(\frac{\pi_{0}}{\pi^{*}}\right)^{\frac{r+\lambda_{2}}{\alpha_{2}}}+ \\
& \bar{p}\left\{\int_{0}^{\frac{1}{\alpha_{2}} \ln \left(\pi^{*} / \pi_{0}\right)} \lambda_{2} e^{-\lambda_{2} \tau_{2}}\left[A\left(\frac{\pi_{0} e^{\alpha_{2} \tau_{2}}}{\pi^{*}}\right)^{\theta_{2}}+C\left(\frac{\pi_{0} e^{\alpha_{2} \tau_{2}}}{\pi^{*}}\right)^{\frac{r}{\alpha_{2}}}\right] e^{-r \tau_{2}} d \tau_{2}\right\} \\
= & (1-\bar{p})\left(\frac{\pi_{0}}{\pi^{*}}\right)^{\frac{r}{\alpha_{2}}}+\frac{\bar{p} \lambda_{2} A}{\lambda_{2}+r-\alpha_{2} \theta_{2}}\left(\frac{\pi_{0}}{\pi^{*}}\right)^{\theta_{2}}- \\
& \frac{\bar{p} \lambda_{2} A}{\lambda_{2}+r-\alpha_{2} \theta_{2}}\left(\frac{\pi_{0}}{\pi^{*}}\right)^{\frac{r+\lambda_{2}}{\alpha_{2}}}+\bar{p} C\left(\frac{\pi_{0}}{\pi^{*}}\right)^{\frac{r}{\alpha_{2}}}-\bar{p} C\left(\frac{\pi_{0}}{\pi^{*}}\right)^{\frac{r+\lambda_{2}}{\alpha_{2}}}+\bar{p}\left(\frac{\pi_{0}}{\pi^{*}}\right)^{\frac{r+\lambda_{2}}{\alpha_{2}}} \\
= & (1-\bar{p}+C \bar{p})\left(\frac{\pi_{0}}{\pi^{*}}\right)^{\frac{r}{\alpha_{2}}}+\frac{\lambda_{2} A \bar{p}}{\lambda_{2}+r-\alpha_{2} \theta_{2}}\left(\frac{\pi_{0}}{\pi^{*}}\right)^{\theta_{2}}+\bar{p}\left(\frac{\lambda_{2}(1-A)+r-\alpha_{2} \theta_{2}}{\lambda_{2}+r-\alpha_{2} \theta_{2}}-C\right)\left(\frac{\pi_{0}}{\pi^{*}}\right)^{\left.\frac{\lambda_{2}+r}{(13}\right)}
\end{aligned}
$$

Note that $\bar{p}=p_{0}$ for $\pi_{0}=\bar{\pi}$ (since $\bar{p}=p\left(\pi_{0} \mid \bar{\pi}\right)$ ), so

$$
\begin{aligned}
\bar{\varphi}_{2}\left(\pi_{0}, \pi^{*}\right)= & \int_{0}^{\infty} \lambda_{1} e^{-\left(r+\lambda_{1}\right) \tau_{1}}\left[\begin{array}{c}
\left(1-p_{0}+C p_{0}\right)\left(\frac{\pi_{0} e^{\alpha_{1} \tau_{1}}}{\pi^{*}}\right)^{\frac{r}{\alpha_{2}}}+\frac{\lambda_{2} A p_{0}}{\lambda_{2}+r-\alpha_{2} \theta_{2}}\left(\frac{\pi_{0} e^{\alpha_{1} \tau_{1}}}{\pi^{*}}\right)^{\theta_{2}} \\
+p_{0}\left(\frac{\lambda_{2}(1-A)+r-\alpha_{2} \theta_{2}}{\lambda_{2}+r-\alpha_{2} \theta_{2}}-C\right)\left(\frac{\pi_{0} e^{\alpha_{1} \tau_{1}}}{\pi^{*}}\right)^{\frac{\lambda_{2}+r}{\alpha_{2}}}
\end{array}\right] d \tau_{1} \\
= & \frac{\lambda_{1}\left(1-p_{0}+C p_{0}\right)}{\lambda_{1}+r\left(1-\frac{\alpha_{1}}{\alpha_{2}}\right)}\left(\frac{\pi_{0}}{\pi^{*}}\right)^{\frac{r}{\alpha_{2}}}+\frac{\lambda_{1} \lambda_{2} A p_{0}}{\left(r+\lambda_{2}-\alpha_{2} \theta_{2}\right)\left(r+\lambda_{1}-\alpha_{1} \theta_{2}\right)}\left(\frac{\pi_{0}}{\pi^{*}}\right)^{\theta_{2}}+ \\
& p_{0}\left[\frac{\lambda_{2}(1-A)+r-\alpha_{2} \theta_{2}}{r+\lambda_{2}-\alpha_{2} \theta_{2}}-C\right]\left(\frac{\pi_{0}}{\pi^{*}}\right)^{\frac{\lambda_{2}+r}{\alpha_{2}}}\left(\frac{\lambda_{1}}{r+\lambda_{1}-\frac{\alpha_{1}}{\alpha_{2}}\left(\lambda_{2}+r\right)}\right)
\end{aligned}
$$

Because we supposed that $\bar{\varphi}_{2}\left(\pi_{0}, \pi^{*}\right)=A\left(\frac{\pi_{0}}{\pi^{*}}\right)^{\theta_{2}}+C\left(\frac{\pi_{0}}{\pi^{*}}\right)^{\frac{r}{\alpha_{2}}}$, we must have that the following three equations hold:

$$
\frac{\lambda_{1}\left(1-p_{0}+C p_{0}\right)}{\lambda_{1}+r\left(1-\frac{\alpha_{1}}{\alpha_{2}}\right)}=C
$$




$$
\frac{\lambda_{1} \lambda_{2} A p_{0}}{\left(r+\lambda_{2}-\alpha_{2} \theta_{2}\right)\left(r+\lambda_{1}-\alpha_{1} \theta_{2}\right)}=A
$$

and

$$
\frac{\lambda_{2}(1-A)+r-\alpha_{2} \theta_{2}}{\lambda_{2}+r-\alpha_{2} \theta_{2}}-C=0 .
$$

Using expression (14) yields that

$$
C=\frac{\lambda_{1}\left(1-p_{0}\right)}{\lambda_{1}\left(1-p_{0}\right)+r\left(1-\frac{\alpha_{1}}{\alpha_{2}}\right)} .
$$

In turn, equation (15) implies that $\theta_{2}$ solves

$$
\left(\lambda_{2}+r-\alpha_{2} \theta_{2}\right)\left(\lambda_{1}+r-\alpha_{1} \theta_{2}\right)=p_{0} \lambda_{1} \lambda_{2}
$$

We have shown before (see (12)) that the roots of this quadratic equation are $\beta_{1}>1$ and $\beta_{2}<0$. Only the negative root can be admissible now, so

$$
\theta_{2}=\beta_{2} \equiv \frac{\alpha_{1}\left(r+\lambda_{1}\right)+\alpha_{2}\left(r+\lambda_{1}\right)+\sqrt{\Delta}}{2 \alpha_{1} \alpha_{2}}<0 .
$$

In addition, equations (16) and (17) with $\theta_{2}=\beta_{2}$ imply that

$$
A=\frac{r\left(1-\frac{\alpha_{1}}{\alpha_{2}}\right)\left(r+\lambda_{2}-\alpha_{2} \beta_{2}\right)}{\lambda_{2}\left[\lambda_{1}\left(1-p_{0}\right)+r\left(1-\frac{\alpha_{1}}{\alpha_{2}}\right)\right]} .
$$

Therefore, recalling that $\phi=\frac{r\left(\alpha_{1}-\alpha_{2}\right)}{r\left(\alpha_{1}-\alpha_{2}\right)-\lambda_{1} \alpha_{2}\left(1-p_{0}\right)}$ yields that

$$
\begin{aligned}
\bar{\varphi}_{2}\left(\pi_{0}, \pi^{*}\right)= & \frac{r\left(\alpha_{1}-\alpha_{2}\right)}{r\left(\alpha_{1}-\alpha_{2}\right)-\lambda_{1} \alpha_{2}\left(1-p_{0}\right)}\left(\frac{r+\lambda_{2}-\alpha_{2} \beta_{2}}{\lambda_{2}}\right)\left(\frac{\pi_{0}}{\pi^{*}}\right)^{\beta_{2}}+ \\
& \left(1-\frac{r\left(\alpha_{1}-\alpha_{2}\right)}{r\left(\alpha_{1}-\alpha_{2}\right)-\lambda_{1} \alpha_{2}\left(1-p_{0}\right)}\right)\left(\frac{\pi_{0}}{\pi^{*}}\right)^{\frac{r}{\alpha_{2}}} \\
= & \frac{\phi\left(r+\lambda_{2}-\alpha_{2} \beta_{2}\right)}{\lambda_{2}}\left(\frac{\pi_{0}}{\pi^{*}}\right)^{\beta_{2}}+(1-\phi)\left(\frac{\pi_{0}}{\pi^{*}}\right)^{\frac{r}{\alpha_{2}}} \\
= & \left(\phi+\delta_{1}-1\right)\left(\frac{\pi_{0}}{\pi^{*}}\right)^{\beta_{2}}+(1-\phi)\left(\frac{\pi_{0}}{\pi^{*}}\right)^{\frac{r}{\alpha_{2}}} .
\end{aligned}
$$


Note that using the definitions of $\phi$ and $\beta_{2}$ implies that

$$
\begin{aligned}
\delta_{1} & =1-\phi+\frac{\phi\left(r+\lambda_{2}-\alpha_{2} \beta_{2}\right)}{\lambda_{2}} \\
& =\frac{r\left(\alpha_{1}-\alpha_{2}\right)\left[\frac{\alpha_{1}\left(r+\lambda_{2}\right)-\alpha_{2}\left(r+\lambda_{1}\right)-\sqrt{\Delta}}{2 \alpha_{1} \lambda_{2}}\right]-\lambda_{1} \alpha_{2}\left(1-p_{0}\right)}{r\left(\alpha_{1}-\alpha_{2}\right)-\lambda_{1} \alpha_{2}\left(1-p_{0}\right)} \in(0,1) .
\end{aligned}
$$

Finally, making use of (13) and (16) with $\theta_{2}=\beta_{2}$, together with expressions (17) and (18), yields that

$$
\underline{\varphi}_{2}\left(\pi_{0}, \pi^{*} \mid \bar{\pi}\right)=\phi p\left(\pi_{0} \mid \bar{\pi}\right)\left(\frac{\pi_{0}}{\pi^{*}}\right)^{\beta_{2}}+\left(1-\phi p\left(\pi_{0} \mid \bar{\pi}\right)\right)\left(\frac{\pi_{0}}{\pi^{*}}\right)^{\frac{r}{\alpha_{2}}}
$$

Proof of Theorem 4. Let $\overline{\mathcal{E}}\left(\pi_{0}, \pi^{*}\right)$ denote the expected value of the stream of discounted profits collected while the process transitions from $\pi_{0}$ until it first hits $\pi^{*}$ from above, conditional on the process being in a growth phase and on the current state being $\pi_{0} \geq \pi^{*}$. Also, let $\underline{\mathcal{E}}\left(\pi_{0}, \pi^{*} \mid \bar{\pi}\right)$ be the expected stream of discounted profits collected while the process transitions from $\pi_{0}$ until it first hits $\pi^{*}$ from above, conditional on the process being in a decline phase that started at level $\bar{\pi}$ and on the current state being $\pi_{0} \in\left(\pi^{*}, \bar{\pi}\right]$. By definition, we have:

$$
\overline{\mathcal{E}}\left(\pi_{0}, \pi^{*}\right)=\int_{0}^{\infty} \lambda_{1} e^{-\lambda_{1} \tau_{1}}\left[\int_{0}^{\tau_{1}} \pi_{0} e^{\alpha_{1} s} e^{-r s} d s+\underline{\mathcal{E}}\left(\pi_{0} e^{\alpha_{1} \tau_{1}}, \pi^{*} \mid \pi_{0} e^{\alpha_{1} \tau_{1}}\right) e^{-r \tau_{1}}\right] d \tau_{1}
$$

and

$$
\begin{aligned}
& \underline{\mathcal{E}}\left(\pi_{0}, \pi^{*} \mid \bar{\pi}\right)=\left(1-p\left(\pi_{0} \mid \bar{\pi}\right)\right)\left(\int_{0}^{\frac{1}{\alpha_{2}} \ln \left(\pi^{*} / \pi_{0}\right)} \pi_{0} e^{\alpha_{2} s} e^{-r s} d s\right)+ \\
& p\left(\pi_{0} \mid \bar{\pi}\right)\left[\begin{array}{c}
\int_{0}^{\frac{1}{\alpha_{2}} \ln \left(\pi^{*} / \pi_{0}\right)} \lambda_{2} e^{-\lambda_{2} \tau_{2}}\left[\int_{0}^{\tau_{2}} \pi_{0} e^{\alpha_{2} s} e^{-r s} d s+\overline{\mathcal{E}}\left(\pi_{0} e^{\alpha_{2} \tau_{2}}, \pi^{*}\right) e^{-r \tau_{2}}\right] d \tau_{2}+ \\
\int_{\frac{1}{\alpha_{2}} \ln \left(\pi^{*} / \pi_{0}\right)}^{\infty} \lambda_{2} e^{-\lambda_{2} \tau_{2}}\left(\int_{0}^{\frac{1}{\alpha_{2}} \ln \left(\pi^{*} / \pi_{0}\right)} \pi_{0} e^{\alpha_{2} s} e^{-r s} d s\right) d \tau_{2}
\end{array}\right] .
\end{aligned}
$$

To see how $\overline{\mathcal{E}}\left(\pi_{0}, \pi^{*}\right)$ arises, note that the process will start declining at some random future date $\tau_{1}$, so the firm gets a stream of discounted profits since the current date until then, and also the discounted value of an asset worth $\underline{\mathcal{E}}\left(\pi_{0} e^{\alpha_{1} \tau_{1}}, \pi^{*} \mid \pi_{0} e^{\alpha_{1} \tau_{1}}\right)$. As for $\underline{\mathcal{E}}\left(\pi_{0}, \pi^{*} \mid \bar{\pi}\right)$, we have that with updated probability $1-p\left(\pi_{0} \mid \bar{\pi}\right)$ the process never grows again, and hence it hits $\pi^{*}$ from above for sure; in the meantime, the firm collects a stream of profits that has to be properly discounted. With complementary probability $p\left(\pi_{0} \mid \bar{\pi}\right)$, the process grows at some random future date $\tau_{2}$, although it may start growing before hitting $\pi^{*}$ (from above). 
If it does not, then the firm reaps a stream of discounted profits until $\pi^{*}$ is hit at time $\frac{\ln \left(\pi^{*} / \pi_{0}\right)}{\alpha_{2}}$. If the process starts growing before hitting $\pi^{*}$, then the firm reaps a discounted profit stream until the process stops declining, and the discounted value of an asset worth $\overline{\mathcal{E}}\left(\pi_{0} e^{\alpha_{2} \tau_{2}}, \pi^{*}\right)$ at such switching date.

Let us suppose that $\overline{\mathcal{E}}\left(\pi_{0}, \pi^{*}\right)=E\left(\pi_{0}\right)^{\theta_{6}}+F\left(\pi_{0}\right)^{\theta_{7}}+G\left(\pi_{0}\right)^{\theta_{8}}$ (where $\theta_{6}, \theta_{7}$ and $\theta_{8}$ are constants, whereas $E, F$ and $G$ do not depend on $\pi_{0}$ although they may depend on $\pi^{*}$ ), so that letting $\bar{p}=p\left(\pi_{0} \mid \bar{\pi}\right)$, plugging the assumed functional form of $\overline{\mathcal{E}}\left(\pi_{0}, \pi^{*}\right)$ into (20) and performing several manipulations yields:

$$
\begin{aligned}
& \underline{\mathcal{E}}\left(\pi_{0}, \pi^{*} \mid \bar{\pi}\right)=\frac{(1-\bar{p}) \pi_{0}\left(1-\left(\frac{\pi_{0}}{\pi^{*}}\right)^{\frac{\left(r-\alpha_{2}\right)}{\alpha_{2}}}\right)}{r-\alpha_{2}}+\frac{\bar{p} \pi_{0}\left(1-\left(\frac{\pi_{0}}{\pi^{*}}\right)^{\frac{\left(r-\alpha_{2}\right)}{\alpha_{2}}}\right)\left(\frac{\pi_{0}}{\pi^{*}}\right)^{\frac{\lambda_{2}}{\alpha_{2}}}}{r-\alpha_{2}}+ \\
& \frac{\bar{p} \pi_{0}}{r+\lambda_{2}-\alpha_{2}}-\frac{\bar{p} \pi_{0}\left(\frac{\pi_{0}}{\pi^{*}}\right)^{\frac{\lambda_{2}}{\alpha_{2}}}}{r-\alpha_{2}}+\frac{\bar{p} \lambda_{2} \pi_{0}\left(\frac{\pi_{0}}{\pi^{*}}\right)^{\frac{\left(r+\lambda_{2}-\alpha_{2}\right)}{\alpha_{2}}}}{\left(r-\alpha_{2}\right)\left(r+\lambda_{2}-\alpha_{2}\right)}+ \\
& \frac{\bar{p} \lambda_{2} E \pi_{0}^{\theta_{6}}\left(1-\left(\frac{\pi_{0}}{\pi^{*}}\right)^{\frac{\left(r+\lambda_{2}-\alpha_{2} \theta_{6}\right)}{\alpha_{2}}}\right)}{\left(r+\lambda_{2}-\alpha_{2} \theta_{6}\right)}+\frac{\bar{p} \lambda_{2} F \pi_{0}^{\theta_{7}}\left(1-\left(\frac{\pi_{0}}{\pi^{*}}\right)^{\frac{\left(r+\lambda_{2}-\alpha_{2} \theta_{7}\right)}{\alpha_{2}}}\right)}{\left(r+\lambda_{2}-\alpha_{2} \theta_{7}\right)}+ \\
& \frac{\bar{p} \lambda_{2} G \pi_{0}^{\theta_{8}}\left(1-\left(\frac{\pi_{0}}{\pi^{*}}\right)^{\frac{\left(r+\lambda_{2}-\alpha_{2} \theta_{8}\right)}{\alpha_{2}}}\right)}{\left(r+\lambda_{2}-\alpha_{2} \theta_{8}\right)} \\
& =\left(\frac{1-\bar{p}}{r-\alpha_{2}}+\frac{\bar{p}}{r+\lambda_{2}-\alpha_{2}}\right) \pi_{0}-\frac{(1-\bar{p})\left(\pi^{*}\right)^{-\frac{\left(r-\alpha_{2}\right)}{\alpha_{2}}}\left(\pi_{0}\right)^{\frac{r}{\alpha_{2}}}}{r-\alpha_{2}}+ \\
& \frac{\bar{p} \lambda_{2} E \pi_{0}^{\theta_{6}}}{r+\lambda_{2}-\alpha_{2} \theta_{6}}+\frac{\bar{p} \lambda_{2} F \pi_{0}^{\theta_{7}}}{r+\lambda_{2}-\alpha_{2} \theta_{7}}+\frac{\bar{p} \lambda_{2} G \pi_{0}^{\theta_{8}}}{r+\lambda_{2}-\alpha_{2} \theta_{8}}-
\end{aligned}
$$

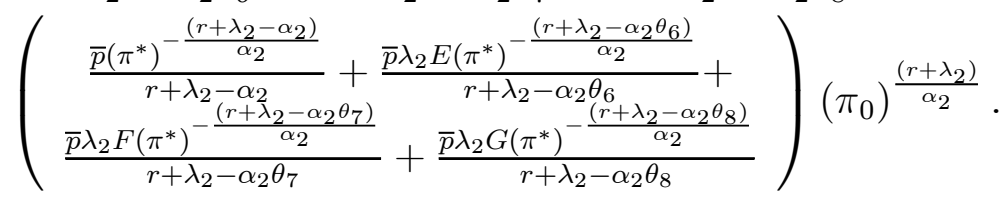

Substitute this into (19) taking into account that $p\left(\pi_{0} e^{\alpha_{1} \tau_{1}} \mid \pi_{0} e^{\alpha_{1} \tau_{1}}\right)=p_{0}$ so as to get the 
following after some manipulations:

$$
\begin{aligned}
& \overline{\mathcal{E}}\left(\pi_{0}, \pi^{*}\right)=\left(1+\frac{\left(1-p_{0}\right) \lambda_{1}}{\left(r-\alpha_{2}\right)}+\frac{p_{0} \lambda_{1}}{r+\lambda_{2}-\alpha_{2}}\right) \frac{\pi_{0}}{\left(r+\lambda_{1}-\alpha_{1}\right)}- \\
& \frac{p_{0} \lambda_{1} \alpha_{2}\left(\pi_{0}\right)^{\frac{\left(r+\lambda_{2}\right)}{\alpha_{2}}}}{\left(\alpha_{2}\left(r+\lambda_{1}\right)-\alpha_{1}\left(r+\lambda_{2}\right)\right)}\left(\begin{array}{c}
\frac{\left(\pi^{*}\right)^{-\frac{\left(r+\lambda_{2}-\alpha_{2}\right)}{\alpha_{2}}}}{\left(r+\lambda_{2}-\alpha_{2}\right)}+\frac{\lambda_{2} E\left(\pi^{*}\right)^{-\frac{\left(r+\lambda_{2}-\alpha_{2} \theta_{6}\right)}{\alpha_{2}}}}{\left(r+\lambda_{2}-\alpha_{2} \theta_{6}\right)}+ \\
\frac{\lambda_{2} F\left(\pi^{*}\right)^{-\frac{\left(r+\lambda_{2}-\alpha_{2} \theta_{7}\right)}{\alpha_{2}}}}{\left(r+\lambda_{2}-\alpha_{2} \theta_{7}\right)}+\frac{\lambda_{2} G\left(\pi^{*}\right)^{-\frac{\left(r+\lambda_{2}-\alpha_{2} \theta_{8}\right)}{\alpha_{2}}}}{\left(r+\lambda_{2}-\alpha_{2} \theta_{8}\right)}
\end{array}\right)- \\
& \frac{\left(1-p_{0}\right)\left(\pi^{*}\right)^{-\frac{\left(r-\alpha_{2}\right)}{\alpha_{2}}} \lambda_{1} \alpha_{2}\left(\pi_{0}\right)^{\frac{r}{\alpha_{2}}}}{\left(r-\alpha_{2}\right)\left(\alpha_{2}\left(r+\lambda_{1}\right)-r \alpha_{1}\right)}+\frac{p_{0} \lambda_{2} E\left(\pi_{0}\right)^{\theta_{6}} \lambda_{1}}{\left(r+\lambda_{2}-\alpha_{2} \theta_{6}\right)\left(r+\lambda_{1}-\alpha_{1} \theta_{6}\right)}+ \\
& \frac{p_{0} \lambda_{2} F\left(\pi_{0}\right)^{\theta_{7}} \lambda_{1}}{\left(r+\lambda_{2}-\alpha_{2} \theta_{7}\right)\left(r+\lambda_{1}-\alpha_{1} \theta_{7}\right)}+\frac{p_{0} \lambda_{2} G\left(\pi_{0}\right)^{\theta_{8}} \lambda_{1}}{\left(r+\lambda_{2}-\alpha_{2} \theta_{8}\right)\left(r+\lambda_{1}-\alpha_{1} \theta_{8}\right)} .
\end{aligned}
$$

Assume that $\theta_{6}=1$ and $\theta_{7}=\frac{r}{\alpha_{2}}$ so that the assumption that $\overline{\mathcal{E}}\left(\pi_{0}, \pi^{*}\right)=E\left(\pi_{0}\right)^{\theta_{6}}+$ $F\left(\pi_{0}\right)^{\theta_{7}}+G\left(\pi_{0}\right)^{\theta_{8}}$ implies that the following must hold:

$$
\begin{gathered}
E=\frac{\left(r+\lambda_{2}-\alpha_{2}\right)\left(r+\lambda_{1}-\alpha_{2}\right)-p_{0} \lambda_{1} \lambda_{2}}{\left(r-\alpha_{2}\right)\left(\left(r+\lambda_{2}-\alpha_{2}\right)\left(r+\lambda_{1}-\alpha_{1}\right)-p_{0} \lambda_{1} \lambda_{2}\right)}=\rho_{1}, \\
F=\frac{\left(1-p_{0}\right) \alpha_{2} \lambda_{1}\left(\pi^{*}\right)^{-\frac{\left(r-\alpha_{2}\right)}{\alpha_{2}}}}{\left(r-\alpha_{2}\right)\left[r\left(\alpha_{1}-\alpha_{2}\right)-\lambda_{1} \alpha_{2}\left(1-p_{0}\right)\right]}, \\
G=-\frac{\left(r+\lambda_{2}-\alpha_{2} \theta_{8}\right)}{\lambda_{2}\left(\pi^{*}\right)^{\left(\theta_{8}-1\right)}}\left(\begin{array}{c}
\frac{1+\lambda_{2} \rho_{1}}{r+\lambda_{2}-\alpha_{2}}+ \\
\frac{\left(1-p_{0}\right) \alpha_{2} \lambda_{1}}{\left(r-\alpha_{2}\right)\left[r\left(\alpha_{1}-\alpha_{2}\right)-\lambda_{1} \alpha_{2}\left(1-p_{0}\right)\right]}
\end{array}\right),
\end{gathered}
$$

and

$$
\left(r+\lambda_{2}-\alpha_{2} \theta_{8}\right)\left(r+\lambda_{1}-\alpha_{1} \theta_{8}\right)=p_{0} \lambda_{1} \lambda_{2}
$$

We must clearly have that $\theta_{8}=\beta_{2}<0$, so using this result as well as expressions (21), (22) 
and (23) yields after very laborious manipulations:

$$
\begin{aligned}
& \underline{\mathcal{E}}\left(\pi_{0}, \pi^{*} \mid \bar{\pi}\right)=\left(\frac{1-\bar{p}}{r-\alpha_{2}}+\frac{\bar{p}\left(1+\lambda_{2} \rho_{1}\right)}{r+\lambda_{2}-\alpha_{2}}\right) \pi_{0}-\frac{(1-\bar{p})\left(\pi^{*}\right)^{-\frac{\left(r-\alpha_{2}\right)}{\alpha_{2}}}\left(\pi_{0}\right)^{\frac{r}{\alpha_{2}}}}{r-\alpha_{2}}- \\
& \left(\begin{array}{c}
\frac{\left(\pi^{*}\right)^{-\frac{\left(r+\lambda_{2}-\alpha_{2}\right)}{\alpha_{2}}}}{r+\lambda_{2}-\alpha_{2}}+\frac{\lambda_{2} E\left(\pi^{*}\right)^{-\frac{\left(r+\lambda_{2}-\alpha_{2}\right)}{\alpha_{2}}}}{r+\lambda_{2}-\alpha_{2}}+ \\
F\left(\pi^{*}\right)^{-\frac{\left(r+\lambda_{2}-\alpha_{2} \theta_{7}\right)}{\alpha_{2}}}+\frac{\lambda_{2} G\left(\pi^{*}\right)^{-\frac{\left(r+\lambda_{2}-\alpha_{2} \beta_{2}\right)}{\alpha_{2}}}}{r+\lambda_{2}-\alpha_{2} \beta_{2}}
\end{array}\right) \bar{p}\left(\pi_{0}\right)^{\frac{\left(r+\lambda_{2}\right)}{\alpha_{2}}}+ \\
& \bar{p} F\left(\pi_{0}\right)^{\frac{r}{\alpha_{2}}}+\frac{\bar{p} \lambda_{2} G \pi_{0}^{\beta_{2}}}{r+\lambda_{2}-\alpha_{2} \beta_{2}} \\
& =\rho_{2}\left(\pi_{0}, \bar{\pi}\right) \pi_{0}-\left[\frac{r\left(\alpha_{1}-\alpha_{2}\right)(1-\bar{p})-\lambda_{1} \alpha_{2}\left(1-p_{0}\right)}{r\left(\alpha_{1}-\alpha_{2}\right)-\lambda_{1} \alpha_{2}\left(1-p_{0}\right)}\right]\left(\frac{\pi_{0}}{r-\alpha_{2}}\right)\left(\frac{\pi_{0}}{\pi^{*}}\right)^{\frac{r-\alpha_{2}}{\alpha_{2}}}- \\
& \bar{p} \pi_{0}\left(\frac{\pi_{0}}{\pi^{*}}\right)^{\beta_{2}-1}\left(\begin{array}{c}
\frac{1+\lambda_{2} \rho_{1}}{r+\lambda_{2}-\alpha_{2}}+ \\
\frac{\left(1-p_{0}\right) \alpha_{2} \lambda_{1}}{\left(r-\alpha_{2}\right)\left[r\left(\alpha_{1}-\alpha_{2}\right)-\lambda_{1} \alpha_{2}\left(1-p_{0}\right)\right]}
\end{array}\right) \\
& =\rho_{2}\left(\pi_{0}, \bar{\pi}\right) \pi_{0}-\left(\frac{r\left(\alpha_{1}-\alpha_{2}\right)(1-\bar{p})-\lambda_{1} \alpha_{2}\left(1-p_{0}\right)}{r\left(\alpha_{1}-\alpha_{2}\right)-\lambda_{1} \alpha_{2}\left(1-p_{0}\right)}\right)\left(\frac{\pi_{0}}{r-\alpha_{2}}\right)\left(\frac{\pi_{0}}{\pi^{*}}\right)^{\frac{r-\alpha_{2}}{\alpha_{2}}}- \\
& \frac{\pi_{0}\left(\frac{\pi_{0}}{\pi^{*}}\right)^{\beta_{2}-1}}{r-\alpha_{2}}\left(\begin{array}{c}
\frac{\lambda_{2}\left(\alpha_{1}-\alpha_{2}\right) \bar{p}}{\left(r+\lambda_{2}-\alpha_{2}\right)\left(r+\lambda_{1}-\alpha_{1}\right)-p_{0} \lambda_{1} \lambda_{2}}+ \\
\frac{r\left(\alpha_{1}-\alpha_{2}\right) \bar{p}}{r\left(\alpha_{1}-\alpha_{2}\right)-\lambda_{1} \alpha_{2}\left(1-p_{0}\right)}
\end{array}\right) \\
& =\rho_{2}\left(\pi_{0}, \bar{\pi}\right) \pi_{0}\left[1-\left(\frac{\pi_{0}}{\pi^{*}}\right)^{\beta_{2}-1}\right]- \\
& \left(\frac{\pi_{0}}{r-\alpha_{2}}\right)\left(\frac{r\left(\alpha_{1}-\alpha_{2}\right)(1-\bar{p})-\lambda_{1} \alpha_{2}\left(1-p_{0}\right)}{r\left(\alpha_{1}-\alpha_{2}\right)-\lambda_{1} \alpha_{2}\left(1-p_{0}\right)}\right)\left[\left(\frac{\pi_{0}}{\pi^{*}}\right)^{\frac{\left(r-\alpha_{2}\right)}{\alpha_{2}}}-\left(\frac{\pi_{0}}{\pi^{*}}\right)^{\beta_{2}-1}\right],
\end{aligned}
$$

where the second equality makes use of the fact that

$$
\frac{\left(\pi^{*}\right)^{-\frac{\left(r+\lambda_{2}-\alpha_{2}\right)}{\alpha_{2}}}}{r+\lambda_{2}-\alpha_{2}}+\frac{\lambda_{2} E\left(\pi^{*}\right)^{-\frac{\left(r+\lambda_{2}-\alpha_{2}\right)}{\alpha_{2}}}}{r+\lambda_{2}-\alpha_{2}}+F\left(\pi^{*}\right)^{-\frac{\left(r+\lambda_{2}-\alpha_{2} \theta_{7}\right)}{\alpha_{2}}}+\frac{\lambda_{2} G\left(\pi^{*}\right)^{-\frac{\left(r+\lambda_{2}-\alpha_{2} \beta_{2}\right)}{\alpha_{2}}}}{r+\lambda_{2}-\alpha_{2} \beta_{2}}=0
$$

the third equality uses

$$
\frac{1+\lambda_{2} \rho_{1}}{r+\lambda_{2}-\alpha_{2}}=\frac{1}{r-\alpha_{2}}+\frac{\lambda_{2}\left(\alpha_{1}-\alpha_{2}\right)}{\left(r-\alpha_{2}\right)\left[\left(r+\lambda_{2}-\alpha_{2}\right)\left(r+\lambda_{1}-\alpha_{1}\right)-p_{0} \lambda_{1} \lambda_{2}\right]}
$$

and the last equality follows because

$$
\frac{\lambda_{2}\left(\alpha_{1}-\alpha_{2}\right) \bar{p}}{\left(r-\alpha_{2}\right)\left[\left(r+\lambda_{1}-\alpha_{1}\right)\left(r+\lambda_{2}-\alpha_{2}\right)-p_{0} \lambda_{1} \lambda_{2}\right]}=\rho_{2}\left(\pi_{0}, \bar{\pi}\right)-\frac{1}{r-\alpha_{2}} .
$$


As a result, the desired expression for $\underline{\mathcal{E}}\left(\pi_{0}, \pi^{*} \mid \bar{\pi}\right)$ follows by simply using

$$
\phi=\frac{r\left(\alpha_{1}-\alpha_{2}\right)}{r\left(\alpha_{1}-\alpha_{2}\right)-\lambda_{1} \alpha_{2}\left(1-p_{0}\right)}
$$

In turn, note that

$$
\begin{aligned}
\overline{\mathcal{E}}\left(\pi_{0}, \pi^{*}\right)= & E \pi_{0}+F\left(\pi_{0}\right)^{\frac{r}{\alpha_{2}}}+G\left(\pi_{0}\right)^{\beta_{2}} \\
= & \rho_{1} \pi_{0}+\frac{\lambda_{1} \alpha_{2}\left(1-p_{0}\right) \pi_{0}}{\left(r-\alpha_{2}\right)\left[r\left(\alpha_{1}-\alpha_{2}\right)-\lambda_{1} \alpha_{2}\left(1-p_{0}\right)\right]}\left(\frac{\pi_{0}}{\pi^{*}}\right)^{\frac{r-\alpha_{2}}{\alpha_{2}}}- \\
& \frac{\left(r+\lambda_{2}-\alpha_{2} \beta_{2}\right) \pi_{0}}{\lambda_{2}}\left(\begin{array}{c}
\frac{1+\lambda_{2} \rho_{1}}{r+\lambda_{2}-\alpha_{2}}+ \\
\frac{\lambda_{1} \alpha_{2}\left(1-p_{0}\right)}{\left(r-\alpha_{2}\right)\left[r\left(\alpha_{1}-\alpha_{2}\right)-\lambda_{1} \alpha_{2}\left(1-p_{0}\right)\right]}
\end{array}\right)\left(\frac{\pi_{0}}{\pi^{*}}\right)^{\beta_{2}-1} \\
= & \rho_{1} \pi_{0}-\frac{(1-\phi) \pi_{0}}{r-\alpha_{2}}\left(\frac{\pi_{0}}{\pi^{*}}\right)^{\frac{r-\alpha_{2}}{\alpha_{2}}}+ \\
& \frac{\left(1-\delta_{1}-\phi\right) \pi_{0}}{\phi}\left(\frac{1+\lambda_{2} \rho_{1}}{r+\lambda_{2}-\alpha_{2}}-\frac{1-\phi}{r-\alpha_{2}}\right)\left(\frac{\pi_{0}}{\pi^{*}}\right)^{\beta_{2}-1}
\end{aligned}
$$

where the last equality makes use of the definition of $\delta_{1}$.

\section{Appendix B}

\section{Proof of Lemma 2.}

Let $\bar{\pi}_{E}^{*}$ denote the threshold that triggers investment when the process is above or at such state for the first time given that the market is growing. In addition, let $\underline{\pi}_{E}^{*}$ denote the threshold that triggers investment when the process is above or at such state for the first time given that the market is declining. We claim that $\underline{\pi}_{E}^{*} \geq \bar{\pi}_{E}^{*},{ }^{35}$ so suppose to the contrary that $\underline{\pi}_{E}^{*}<\bar{\pi}_{E}^{*}$, and consider states such that the firm does not invest immediately if the market is in growth, but such that the arrival of the next switching date would trigger immediate investment: formally, $\pi_{0} \in\left[\underline{\pi}_{E}^{*}, \bar{\pi}_{E}^{*}\right)$. In this case, the dynamics of the value of the investment opportunity while the market is growing, denoted by $\bar{V}_{E}^{*}\left(\pi_{0}\right)$, are given by the following Bellman equation:

$\bar{V}_{E}^{*}\left(\pi_{0}\right)=\max \left\{\rho_{1} \pi_{0}-K,(1-r d t)\left[\lambda_{1} d t\left(\rho_{2}\left(\pi_{0}+\alpha_{1} \pi_{0} d t\right)-K\right)+\left(1-\lambda_{1} d t\right) \bar{V}_{E}^{*}\left(\pi_{0}+\alpha_{1} \pi_{0} d t\right)\right]\right\}$.

On the waiting region, a Taylor expansion and straightforward manipulations ignoring terms

\footnotetext{
${ }^{35}$ Note that this implies that the firm invests only if the market is growing owing to the continuous sample path of the stochastic process.
} 
of order higher than $d t$ yield

$$
\left(r+\lambda_{1}\right) \bar{V}_{E}^{*}\left(\pi_{0}\right)=\alpha_{1} \pi_{0} \frac{d \bar{V}_{E}^{*}\left(\pi_{0}\right)}{d \pi_{0}}+\lambda_{1}\left(\rho_{2} \pi_{0}-K\right)
$$

The solution to this differential equation is

$$
\bar{V}_{E}^{*}\left(\pi_{0}\right)=\frac{\lambda_{1} \rho_{2} \pi_{0}}{r+\lambda_{1}-\alpha_{1}}-\frac{\lambda_{1} K}{r+\lambda_{1}}+B\left(\pi_{0}\right)^{\frac{r+\lambda_{1}}{\alpha_{1}}}
$$

where $B$ is a constant. In particular, we know that $\bar{V}_{E}^{*}\left(\bar{\pi}_{E}^{*}\right)=\rho_{1} \bar{\pi}_{E}^{*}-K$, whence one can get the value of $B$ and plug it into expression (25) so as to get

$$
\bar{V}_{E}^{*}\left(\pi_{0} \mid \bar{\pi}_{E}^{*}\right)=\frac{\lambda_{1} \rho_{2} \pi_{0}}{r+\lambda_{1}-\alpha_{1}}-\frac{\lambda_{1} K}{r+\lambda_{1}}+\left[\rho_{1} \bar{\pi}_{E}^{*}-K+\frac{\lambda_{1} K}{r+\lambda_{1}}-\frac{\lambda_{1} \rho_{2} \bar{\pi}_{E}^{*}}{r+\lambda_{1}-\alpha_{1}}\right]\left(\frac{\pi_{0}}{\bar{\pi}_{E}^{*}}\right)^{\frac{r+\lambda_{1}}{\alpha_{1}}}
$$

Using the fact that

$$
\left(r+\lambda_{1}-\alpha_{1}\right) \rho_{1}=1+\lambda_{1} \rho_{2}
$$

and maximizing $\bar{V}_{E}^{*}\left(\pi_{0} \mid \bar{\pi}_{E}^{*}\right)$ with respect to $\bar{\pi}_{E}^{*}$ yields that $\bar{\pi}_{E}^{*}=r K$ (since $\bar{V}_{E}^{*}\left(\pi_{0} \mid \bar{\pi}_{E}^{*}\right)$ is strictly quasi-concave). As a result, we have that the value of an optimally managed investment opportunity if the market is growing at $\pi_{0} \in\left[\underline{\pi}_{E}^{*}, \bar{\pi}_{E}^{*}\right)$ equals

$$
\bar{V}_{E}^{*}\left(\pi_{0} \mid r K\right)=\frac{\lambda_{1} \rho_{2} \pi_{0}}{r+\lambda_{1}-\alpha_{1}}-\frac{\lambda_{1} K}{r+\lambda_{1}}+\frac{\alpha_{1} r K}{\left(r+\lambda_{1}-\alpha_{1}\right)\left(r+\lambda_{1}\right)}\left(\frac{\pi_{0}}{r K}\right)^{\frac{r+\lambda_{1}}{\alpha_{1}}} .
$$

Once the value of $\bar{\pi}_{E}^{*}$ has been found out, it simply remains to find out that of $\underline{\pi}_{E}^{*}$. To this end, let us examine the value of the investment opportunity in a growing market for $\pi_{0}<\underline{\pi}_{E}^{*}$. Let $\underline{V}_{E}^{*}\left(\pi_{0}\right)$ denote the value of the investment opportunity if the market is declining, and note that the dynamics of $\underline{V}_{E}^{*}\left(\pi_{0}\right)$ are given by the following differential equation:

$$
\left(r+\lambda_{2}\right) \underline{V}_{E}^{*}\left(\pi_{0}\right)=\alpha_{2} \pi_{0} \frac{d \underline{V}_{E}^{*}\left(\pi_{0}\right)}{d \pi_{0}}+\lambda_{2} \bar{V}_{E}^{*}\left(\pi_{0}\right)
$$

since the firm is in the waiting region during the decline phase. Similarly, $\bar{V}_{E}^{*}\left(\pi_{0}\right)$ satisfies the following differential equation for $\pi_{0}<\underline{\pi}_{E}^{*}$ :

$$
\left(r+\lambda_{1}\right) \bar{V}_{E}^{*}\left(\pi_{0}\right)=\alpha_{1} \pi_{0} \frac{d \bar{V}_{E}^{*}\left(\pi_{0}\right)}{d \pi_{0}}+\lambda_{1} V_{E}^{-}\left(\pi_{0}\right)
$$

since the firm does not invest right away if the market switches from growth to decline. Solving the system of differential equations comprised by (28) and (29), and using the boundary 
condition that $\bar{V}_{E}^{*}(0)=0$ leads to the following solution:

$$
\bar{V}_{E}^{*}\left(\pi_{0}\right)=R\left(\pi_{0}\right)^{\beta_{1}},
$$

where $R$ is a constant to found out, and $\beta_{1}=\frac{\alpha_{1}\left(r+\lambda_{2}\right)+\alpha_{2}\left(r+\lambda_{1}\right)-\sqrt{\Delta}}{2 \alpha_{1} \alpha_{2}}>1$. Evaluating (27) at $\pi_{0}=\underline{\pi}_{E}^{*}$, we have that the boundary condition from which $R$ can be derived is

$$
R\left(\underline{\pi}_{E}^{*}\right)^{\beta_{1}}=\frac{\lambda_{1} \rho_{2} \underline{\pi}_{E}^{*}}{r+\lambda_{1}-\alpha_{1}}-\frac{\lambda_{1} K}{r+\lambda_{1}}+\frac{\alpha_{1} r K}{\left(r+\lambda_{1}-\alpha_{1}\right)\left(r+\lambda_{1}\right)}\left(\frac{\underline{\pi}_{E}^{*}}{r K}\right)^{\frac{r+\lambda_{1}}{\alpha_{1}}}
$$

so expression (30) becomes

$$
\bar{V}_{E}^{*}\left(\pi_{0} \mid \underline{\pi}_{E}^{*}\right)=\left[\frac{\lambda_{1} \rho_{2} \underline{\pi}_{E}^{*}}{r+\lambda_{1}-\alpha_{1}}-\frac{\lambda_{1} K}{r+\lambda_{1}}+\frac{\alpha_{1} r K}{\left(r+\lambda_{1}-\alpha_{1}\right)\left(r+\lambda_{1}\right)}\left(\frac{\underline{\pi}_{E}^{*}}{r K}\right)^{\frac{r+\lambda_{1}}{\alpha_{1}}}\right]\left(\frac{\pi_{0}}{\underline{\pi}_{E}^{*}}\right)^{\beta_{1}}
$$

Performing some manipulations, we have that the derivative of $\bar{V}_{E}^{*}\left(\pi_{0} \mid \underline{\pi}_{E}^{*}\right)$ with respect to $\underline{\pi}_{E}^{*}$ is

$$
\frac{\partial \bar{V}_{E}^{*}\left(\pi_{0} \mid \underline{\pi}_{E}^{*}\right)}{\partial \underline{\pi}_{E}^{*}}=\left[\frac{\lambda_{1} \rho_{2}\left(1-\beta_{1}\right) \underline{\pi}_{E}^{*}}{r+\lambda_{1}-\alpha_{1}}+\frac{r K\left(r+\lambda_{1}-\alpha_{1} \beta_{1}\right)}{\left(r+\lambda_{1}-\alpha_{1}\right)\left(r+\lambda_{1}\right)}\left(\frac{\underline{\pi}_{E}^{*}}{r K}\right)^{\frac{r+\lambda_{1}}{\alpha_{1}}}+\frac{\beta_{1} \lambda_{1} K}{r+\lambda_{1}}\right] \frac{\left(\pi_{0}\right)^{\beta_{1}}}{\left(\underline{\pi}_{E}^{*}\right)^{\beta_{1}+1}} .
$$

We claim that $\frac{\partial^{2} \bar{V}_{E}^{*}\left(\pi_{0} \mid \underline{\pi}_{E}^{*}\right)}{\partial\left(\underline{\pi}_{E}^{*}\right)^{2}}<0$ whenever $\frac{\partial \bar{V}_{E}^{*}\left(\pi_{0} \mid \underline{\pi}_{E}^{*}\right)}{\partial \underline{\pi}_{E}^{*}}=0$ holds (i.e., we claim that $\bar{V}_{E}^{*}\left(\pi_{0} \mid \underline{\pi}_{E}^{*}\right)$ is strictly quasi-concave). For the value of $\underline{\pi}_{E}^{*}$ such that $\frac{\partial \bar{V}_{E}^{*}\left(\pi_{0} \mid \underline{\pi}_{E}^{*}\right)}{\partial \underline{\pi}_{E}^{*}}=0$ holds, we have that

$$
\begin{aligned}
\frac{\partial^{2} \bar{V}_{E}^{*}\left(\pi_{0} \mid \underline{\pi}_{E}^{*}\right)}{\partial\left(\underline{\pi}_{E}^{*}\right)^{2}} & =\left[\frac{\lambda_{1} \rho_{2}\left(1-\beta_{1}\right)}{r+\lambda_{1}-\alpha_{1}}+\frac{r K\left(r+\lambda_{1}-\alpha_{1} \beta_{1}\right)}{\alpha_{1}\left(r+\lambda_{1}-\alpha_{1}\right) \underline{\pi}_{E}^{*}}\left(\frac{\underline{\pi}_{E}^{*}}{r K}\right)^{\frac{r+\lambda_{1}}{\alpha_{1}}}\right] \frac{\left(\pi_{0}\right)^{\beta_{1}}}{\left(\underline{\pi}_{E}^{*}\right)^{\beta_{1}+1}} \\
& =\frac{\lambda_{1}\left[\left(\beta_{1}-1\right) \rho_{2} \underline{\pi}_{E}^{*}-\beta_{1} K\right]\left(\pi_{0}\right)^{\beta_{1}}}{\alpha_{1}\left(\underline{\pi}_{E}^{*}\right)^{\beta_{1}+2}} \\
& <\frac{\lambda_{1} \beta_{1}\left(\pi_{0}\right)^{\beta_{1}}}{\alpha_{1}\left(\underline{\pi}_{E}^{*}\right)^{\beta_{1}+2}}\left(\frac{\pi_{E}^{*}-r K}{r}\right)<0,
\end{aligned}
$$

where the last equality follows because $\frac{\partial \bar{V}_{E}^{*}\left(\pi_{0} \mid \underline{\pi}_{E}^{*}\right)}{\partial \underline{\pi}_{E}^{*}}=0$, the first inequality holds because 
$\rho_{2}<\rho_{1}<\frac{\beta_{1}}{r\left(\beta_{1}-1\right)},{ }^{36}$ and the last inequality follows since $\underline{\pi}_{E}^{*}<r K$. Evaluate $\frac{\partial \bar{V}_{E}^{*}\left(\pi_{0} \mid \underline{\pi}_{E}^{*}\right)}{\partial \underline{\pi}_{E}^{*}}$ at $\underline{\pi}_{E}^{*}=r K$ so as to get

$$
\begin{aligned}
\frac{\partial \bar{V}_{E}^{*}\left(\pi_{0} \mid r K\right)}{\partial \underline{\pi}_{E}^{*}}= & {\left[\frac{\lambda_{1} \rho_{2}\left(1-\beta_{1}\right)\left(r+\lambda_{1}\right) r K}{\left(r+\lambda_{1}\right)\left(r+\lambda_{1}-\alpha_{1}\right)}+\frac{r K\left(r+\lambda_{1}-\alpha_{1} \beta_{1}\right)}{\left(r+\lambda_{1}-\alpha_{1}\right)\left(r+\lambda_{1}\right)}+\frac{\beta_{1} \lambda_{1}\left(r+\lambda_{1}-\alpha_{1}\right) K}{\left(r+\lambda_{1}\right)\left(r+\lambda_{1}-\alpha_{1}\right)}\right] \times } \\
& \frac{\left(\pi_{0}\right)^{\beta_{1}}}{\left(\underline{\pi}_{E}^{*}\right)^{\beta_{1}+1}} \\
= & \frac{\left[\left(1-\beta_{1}\right) r \rho_{1}+\beta_{1}\right] K\left(\pi_{0}\right)^{\beta_{1}}}{\left(\underline{\pi}_{E}^{*}\right)^{\beta_{1}+1}}>0,
\end{aligned}
$$

since $\lambda_{1} \rho_{2}=\left(\lambda_{1}+r-\alpha_{1}\right) \rho_{1}-1$ by expression $(26)$ and $\rho_{1}<\frac{\beta_{1}}{r\left(\beta_{1}-1\right)}$ was just shown to always hold. This proves that $\bar{V}_{E}^{*}\left(\pi_{0} \mid \underline{\pi}_{E}^{*}\right)$ is an increasing function for $\underline{\pi}_{E}^{*}<r K$, and (31) implies that the payoff expected by the firm would be bounded above by

$$
\begin{aligned}
\bar{V}_{E}^{*}\left(\pi_{0} \mid r K\right) & =\left[\frac{\lambda_{1} \rho_{2} r K}{r+\lambda_{1}-\alpha_{1}}-\frac{\lambda_{1} K}{r+\lambda_{1}}+\frac{\alpha_{1} r K}{\left(r+\lambda_{1}-\alpha_{1}\right)\left(r+\lambda_{1}\right)}\right]\left(\frac{\pi_{0}}{r K}\right)^{\beta_{1}} \\
& =\left(\rho_{1} r K-K\right)\left(\frac{\pi_{0}}{r K}\right)^{\beta_{1}},
\end{aligned}
$$

which is the (expected) payoff if the firm does not invest during the market decline and it invests the first time the market reaches state $r K$, conditional upon the current state being

${ }^{36}$ To show that $\rho_{1}<\frac{\beta_{1}}{r\left(\beta_{1}-1\right)}$, note that

$$
\left(r+\lambda_{1}-\alpha_{1}\right)\left(r+\lambda_{2}-\alpha_{2}\right)-p_{0} \lambda_{1} \lambda_{2}=\alpha_{1} \alpha_{2}\left(1-\beta_{1}\right)\left(1-\beta_{2}\right),
$$

so some algebraic manipulations yield that $\rho_{1}$ can be rewritten as follows:

$$
\begin{aligned}
\rho_{1} & =\frac{\left(r-\alpha_{2}\right)\left(r+\lambda_{1}+\lambda_{2}-\alpha_{2}\right)+\left(1-p_{0}\right) \lambda_{1} \lambda_{2}}{\alpha_{1} \alpha_{2}\left(r-\alpha_{2}\right)\left(1-\beta_{1}\right)\left(1-\beta_{2}\right)} \\
& =\frac{1}{r}\left[\frac{r\left(r+\lambda_{1}+\lambda_{2}\right)+\left(1-p_{0}\right) \lambda_{1} \lambda_{2}-r \alpha_{2}}{\alpha_{1} \alpha_{2}\left(1-\beta_{1}\right)\left(1-\beta_{2}\right)}+\frac{\left(1-p_{0}\right) \lambda_{1} \lambda_{2}}{\alpha_{1}\left(r-\alpha_{2}\right)\left(1-\beta_{1}\right)\left(1-\beta_{2}\right)}\right] \\
& =\frac{1}{r}\left[\frac{\beta_{1} \beta_{2}-\frac{r}{\alpha_{1}}}{\left(1-\beta_{1}\right)\left(1-\beta_{2}\right)}+\frac{\left(1-p_{0}\right) \lambda_{1} \lambda_{2}}{\alpha_{1}\left(r-\alpha_{2}\right)\left(1-\beta_{1}\right)\left(1-\beta_{2}\right)}\right],
\end{aligned}
$$

where the last equality makes use of the fact that $\alpha_{1} \alpha_{2} \beta_{1} \beta_{2}=r\left(r+\lambda_{1}+\lambda_{2}\right)+\left(1-p_{0}\right) \lambda_{1} \lambda_{2}$. As a result, it follows that

$$
\begin{aligned}
\frac{\left(\beta_{1}-1\right) \rho_{1} r-\beta_{1}}{\rho_{1}\left(\beta_{1}-1\right)} & =\frac{\beta_{1} \beta_{2}-\frac{r}{\alpha_{1}}-\beta_{1}\left(\beta_{2}-1\right)}{\rho_{1}\left(\beta_{1}-1\right)\left(\beta_{2}-1\right)}+\frac{\left(1-p_{0}\right) \lambda_{1} \lambda_{2}}{\alpha_{1} \rho_{1}\left(r-\alpha_{2}\right)\left(\beta_{1}-1\right)\left(\beta_{2}-1\right)} \\
& <\frac{\alpha_{1} \beta_{1}-r}{\alpha_{1} \rho_{1}\left(\beta_{1}-1\right)\left(\beta_{2}-1\right)}<0
\end{aligned}
$$

since $\beta_{1}>1, \beta_{2}<0$ and $\alpha_{1} \beta_{1}-r=\lambda_{1}\left(1-\delta_{2}\right)>0$. Hence, we must have that $\rho_{1}<\frac{\beta_{1}}{r\left(\beta_{1}-1\right)}$. 
$\pi_{0}<r K$. As we show below (see Proposition 1), the firm's maximal payoff conditional upon investing only if the market is growing is $\max _{\pi_{E}}\left(\rho_{1} \pi_{E}-K\right)\left(\pi_{0} / \pi_{E}\right)^{\beta_{1}}$. The fact that

$$
\bar{V}_{E}^{*}\left(\pi_{0} \mid r K\right)=\left(\rho_{1} r K-K\right)\left(\frac{\pi_{0}}{r K}\right)^{\beta_{1}}<\max _{\pi_{E}}\left(\rho_{1} \pi_{E}-K\right)\left(\frac{\pi_{0}}{\pi_{E}}\right)^{\beta_{1}}
$$

contradicts the optimality of investing during the market decline, which concludes the proof.

Proof of Proposition 2. Note that expression (2) holds if and only if

$$
\rho_{1}\left(\alpha_{1} \beta_{1}-\alpha_{1}\right) \pi_{E}^{*}=\alpha_{1} \beta_{1} K
$$

is satisfied. Because we have that $\alpha_{1} \beta_{1}=\lambda_{1}\left(1-\delta_{2}\right)+r$, condition (32) is equivalent to

$$
\left(r-\alpha_{1}\right) \rho_{1} \pi_{E}^{*}=r K+\lambda_{1}\left(1-\delta_{2}\right)\left(K-\rho_{1} \pi_{E}^{*}\right)
$$

It is simple to show that it holds that $\left(r-\alpha_{1}\right) \rho_{1}=1+\lambda_{1}\left(\rho_{2}-\rho_{1}\right)$, so plugging this equality into the previous expression yields the desired result after canceling some terms and rearranging:

$$
\pi_{E}^{*}=r K+\lambda_{1}\left[\delta_{2}\left(\rho_{1} \pi_{E}^{*}-K\right)-\left(\rho_{2} \pi_{E}^{*}-K\right)\right] .
$$

Proof of Proposition 4. We solve the firm's dynamic optimization problem by working backwards. If the declining phase of cycle $N$ is ever reached, then the firm knows that there will be no growth phase in the future. Letting $\pi_{0}$ be the profit level that an active firm makes at the current date, and denoting the level at which it chooses to exit by $\underline{\pi}_{N}$, we have that the optimal exit threshold is given by the maximization of the following function with respect to $\underline{\pi}_{N}$ :

$$
\underline{V}_{N}\left(\underline{\pi}_{N} \mid \pi_{0}\right)=\int_{0}^{\frac{1}{\alpha_{2}} \ln \left(\underline{\pi}_{N} / \pi_{0}\right)} \pi_{0} e^{\alpha_{2} s} e^{-r s} d s+S\left(\frac{\pi_{0}}{\underline{\pi}_{N}}\right)^{\frac{r}{\alpha_{2}}} .
$$

Hence, the firm earns a discounted profit stream in a contracting market until level $\underline{\pi}_{N}$ is first hit; at such date, the firm exits irreversibly and seizes the discounted value of the outside option.

Accounting for corner solutions, it is simple to show that the optimal disinvestment rule calls for exiting the first time the process is below level $\underline{\pi}_{N}^{*} \equiv \min \left(r S, \pi_{0}\right)$. Hence, letting $\underline{V}_{N}^{*}\left(\pi_{0}\right) \equiv \underline{V}_{N}\left(\underline{\pi}_{N}^{*} \mid \pi_{0}\right)$ denote the value of an optimally managed disinvestment opportunity given state $\pi_{0}$, we have that the firm's value in the declining phase of cycle $N$ when the state 
is $\pi_{0}$ is:

$$
\underline{V}_{N}^{*}\left(\pi_{0}\right)=\left\{\begin{array}{cl}
\frac{\pi_{0}}{r-\alpha_{2}}-\frac{\alpha_{2} S}{r-\alpha_{2}}\left(\frac{\pi_{0}}{r S}\right)^{\frac{r}{\alpha_{2}}} & \text { if } \pi_{0}>r S \\
S & \text { if } \pi_{0} \leq r S
\end{array} .\right.
$$

Suppose now that the growing phase of cycle $N$ is reached with the firm active in the market. Let $\pi_{0}$ denote the current market state, and denote the exit threshold in the last growing phase by $\bar{\pi}_{N}$. Then the firm must choose a level $\bar{\pi}_{N}$ that maximizes the following function:

$$
\begin{aligned}
\bar{V}_{N}\left(\bar{\pi}_{N} \mid \pi_{0}\right)= & \int_{0}^{\frac{1}{\alpha_{1}} \ln \left(\bar{\pi}_{N} / \pi_{0}\right)} \lambda_{1} e^{-\lambda_{1} \tau_{1}}\left[\int_{0}^{\tau_{1}} \pi_{0} e^{\alpha_{1} s} e^{-r s} d s+\underline{V}_{N}^{*}\left(\pi_{0} e^{\alpha_{1} \tau_{1}}\right) e^{-r \tau_{1}}\right] d \tau_{1}+ \\
& \int_{\frac{1}{\alpha_{1}} \ln \left(\bar{\pi}_{N} / \pi_{0}\right)}^{\infty} \lambda_{1} e^{-\lambda_{1} \tau_{1}}\left[\int_{0}^{\frac{1}{\alpha_{1}} \ln \left(\bar{\pi}_{N} / \pi_{0}\right)} \pi_{0} e^{\alpha_{1} s} e^{-r s} d s+S\left(\frac{\pi_{0}}{\bar{\pi}_{N}}\right)^{\frac{r}{\alpha_{1}}}\right] d \tau_{1} .
\end{aligned}
$$

Therefore, if the market stops growing at the random time $\tau_{1} \in\left(0, \frac{1}{\alpha_{1}} \ln \left(\bar{\pi}_{N} / \pi_{0}\right)\right)$, then the firm collects a discounted profit stream until time $\tau_{1}$, and attains the discounted value of a disinvestment opportunity in a declining market at level $\pi_{0} e^{\alpha_{1} \tau_{1}}$. If the market stops growing at some $\tau_{1} \geq \frac{1}{\alpha_{1}} \ln \left(\bar{\pi}_{N} / \pi_{0}\right)$, then the firm exits at state $\bar{\pi}_{N}$ and hence reaps a stream of discounted profits until such state is first reached, and the properly discounted value of the outside option.

Differentiating $\bar{V}_{N}\left(\bar{\pi}_{N} \mid \pi_{0}\right)$ with respect to $\bar{\pi}_{N}$ and performing some tedious algebra yields that

$$
\frac{\partial \bar{V}_{N}\left(\bar{\pi}_{N} \mid \pi_{0}\right)}{\partial \bar{\pi}_{N}}=\frac{\bar{\pi}_{N}-r S+\lambda_{1}\left(\underline{V}_{N}^{*}\left(\bar{\pi}_{N}\right)-S\right)}{\alpha_{1} \bar{\pi}_{N}}\left(\frac{\pi_{0}}{\bar{\pi}_{N}}\right)^{\frac{r+\lambda_{1}}{\alpha_{1}}}
$$

Therefore, using (33) implies that $\operatorname{sign}\left(\frac{\partial \bar{V}_{N}\left(\bar{\pi}_{N} \mid \pi_{0}\right)}{\partial \bar{\pi}_{N}}\right)=\operatorname{sign}\left(\bar{\pi}_{N}-r S\right)$, and hence the unique value of $\bar{\pi}_{N}$ such that $\partial \bar{V}_{N}\left(\bar{\pi}_{N} \mid \pi_{0}\right) / \partial \bar{\pi}_{N}=0$ is $\bar{\pi}_{N}=r S$. Using the differentiability of $\underline{V}_{N}^{*}\left(\pi_{0}\right)$, it is easy to show that $\partial^{2} \bar{V}_{N}\left(r S \mid \pi_{0}\right) / \partial \bar{\pi}_{N}^{2}=1>0\left(\right.$ since $\left.d \underline{V}_{N}^{*}(r S) / d \pi_{0}=0\right)$, which implies that $\bar{V}_{N}\left(\bar{\pi}_{N} \mid \pi_{0}\right)$ is strictly quasi-convex. As a result, the firm must choose at state $\pi_{0}$ whether to exit immediately and seize $S$ or to wait until the market starts decaying and gain an expected payoff equal to:

$$
\begin{gathered}
\bar{V}_{N}\left(\infty \mid \pi_{0}\right)=\int_{0}^{\frac{1}{\alpha_{1}} \ln \left(\max \left(r S, \pi_{0}\right) / \pi_{0}\right)} \lambda_{1} e^{-\lambda_{1} \tau_{1}}\left(\int_{0}^{\tau_{1}} \pi_{0} e^{\alpha_{1} s} e^{-r s} d s+S e^{-r \tau_{1}}\right) d \tau_{1}+ \\
\int_{\frac{1}{\alpha_{1}} \ln \left(\max \left(r S, \pi_{0}\right) / \pi_{0}\right)}^{\infty} \lambda_{1} e^{-\lambda_{1} \tau_{1}}\left\{\left(\frac{\pi_{0} e^{\alpha_{1} \tau_{1}}}{r-\alpha_{2}}-\frac{\alpha_{2} S}{r-\alpha_{2}}\left(\frac{\pi_{0} e^{\alpha_{1} \tau_{1}}}{r S}\right)^{\frac{r}{\alpha_{2}}}\right) e^{-r \tau_{1}}\right\} d \tau_{1} .
\end{gathered}
$$


It is easy to see that there exists a unique $\bar{\pi}_{N}^{*}<r S$ such that

$$
\bar{V}_{N}^{*}\left(\pi_{0}\right)=\left\{\begin{array}{cc}
\frac{\left(\lambda_{1}+r-\alpha_{2}\right) \pi_{0}}{\left(r-\alpha_{2}\right)\left(\lambda_{1}+r-\alpha_{1}\right)}-\frac{\lambda_{1} \alpha_{2}^{2} S}{\left(r-\alpha_{2}\right)\left[\lambda_{1} \alpha_{2}+r\left(\alpha_{2}-\alpha_{1}\right)\right]}\left(\frac{\pi_{0}}{r S}\right)^{\frac{r}{\alpha_{2}}} & \text { if } \pi_{0}>r S \\
\frac{\pi_{0}}{r+\lambda_{1}-\alpha_{1}}+\frac{\lambda_{1} S}{r+\lambda_{1}}+ & \text { if } \bar{\pi}_{N}^{*}<\pi_{0} \leq r S \\
\frac{\lambda_{1} \alpha_{1}^{2} r S}{\left(r+\lambda_{1}\right)\left(r+\lambda_{1}-\alpha_{1}\right)\left(r\left(\alpha_{1}-\alpha_{2}\right)-\alpha_{2} \lambda_{1}\right)}\left(\frac{\pi_{0}}{r S}\right)^{\frac{r+\lambda_{1}}{\alpha_{1}}} & \text { if } \pi_{0} \leq \bar{\pi}_{N}^{*} \\
S &
\end{array}\right.
$$

where $\bar{V}_{N}^{*}\left(\pi_{0}\right) \equiv \bar{V}_{N}\left(\bar{\pi}_{N}^{*} \mid \pi_{0}\right)$ (and $\bar{\pi}_{N}^{*}$ satisfies $\bar{V}_{N}\left(\infty \mid \bar{\pi}_{N}^{*}\right)=S$ ).

This completes the characterization of the optimal disinvestment policy whenever cycle $N$ is reached. In the set of cycles $N-k$ (where $k$ is an integer weakly greater than 1 ), the optimal disinvestment policy must account for the fact that the firm is unsure about whether the decline phase of the cycle is going to be the permanent or not. In the remainder of the proof, we prove inductively a claim similar to that made in the statement of the proposition, namely:

Claim 1 For each cycle $N-k$ (with $k \geq 1$ ), there exists a unique optimal disinvestment policy, and it is such that the firm exits only if the market is declining. In addition, if the market is in the declining phase of cycle $N-k$ at state $\pi_{0}$ and the decline has started at state $\bar{\pi} \geq \pi_{0}$, then there exists a unique $\widehat{\pi}_{N-k}<r S$ such that the threshold $\underline{\pi}_{N-k}^{*}(\bar{\pi})$ below which exit is optimal satisfies $\underline{\pi}_{N-k}^{*}(\bar{\pi})=\bar{\pi}$ for $\bar{\pi} \leq \widehat{\pi}_{N-k}$ and

$$
r S=\underline{\pi}_{N-k}^{*}(\bar{\pi})+p\left(\underline{\pi}_{N-k}^{*}(\bar{\pi}) \mid \bar{\pi}\right) \lambda_{2}\left[\bar{V}_{N-k+1}^{*}\left(\underline{\pi}_{N-k}^{*}(\bar{\pi})\right)-S\right]
$$

for $\bar{\pi}>\widehat{\pi}_{N-k}$. It holds that $\underline{\pi}_{N-k}^{*}(\bar{\pi})$ is an increasing function that approaches $r S$ as $\bar{\pi} \rightarrow \infty$. Also, it holds that

$$
\bar{V}_{N-k}^{*}\left(\pi_{0}\right)=\left\{\begin{array}{cl}
\bar{V}_{N-k}\left(\infty \mid \pi_{0}\right) & \text { if } \pi_{0}>\bar{\pi}_{N-k}^{*} \\
S & \text { if } \pi_{0} \leq \bar{\pi}_{N-k}^{*}
\end{array}\right.
$$

for some $\bar{\pi}_{N-k}^{*}$, and that $\bar{V}_{N-k+1}^{*}\left(\underline{\pi}_{N-k}^{*}(\bar{\pi})\right)>S$ for all $\bar{\pi}>\widehat{\pi}_{N-k}$, where $\widehat{\pi}_{N-k}$ is uniquely given by $\widehat{\pi}_{N-k}+p_{0} \lambda_{2}\left[\bar{V}_{N-k+1}^{*}\left(\widehat{\pi}_{N-k}\right)-S\right]=r S$.

We start by showing that Claim 1 holds for $k=1$. So suppose that the market is in a declining stage that started at level $\bar{\pi}$, with the current state being $\pi_{0} \leq \bar{\pi}$. Letting $\underline{\pi}_{N-1}$ be the level at which the firm would find it optimal to exit should the market be declining by then, we can compute the firm's expected payoff to exiting at $\underline{\pi}_{N-1}$ using the posterior 
belief in Lemma 1:

$$
\begin{aligned}
& \underline{V}_{N-1}\left(\underline{\pi}_{N-1} \mid \pi_{0}, \bar{\pi}\right)=\left(1-p\left(\pi_{0} \mid \bar{\pi}\right)\right)\left[\int_{0}^{\frac{1}{\alpha_{2}} \ln \left(\underline{\pi}_{N-1} / \pi_{0}\right)} \pi_{0} e^{\alpha_{2} s} e^{-r s} d s+S\left(\frac{\pi_{0}}{\underline{\pi}_{N-1}}\right)^{\frac{r}{\alpha_{2}}}\right]+ \\
& p\left(\pi_{0} \mid \bar{\pi}\right)\left\{\begin{array}{c}
\int_{0}^{\frac{1}{\alpha_{2}} \ln \left(\underline{\pi}_{N-1} / \pi_{0}\right)} \lambda_{2} e^{-\lambda_{2} \tau_{2}}\left[\int_{0}^{\tau} \pi_{0} e^{\alpha_{2} s} e^{-r s} d s+\bar{V}_{N}^{*}\left(\pi_{0} e^{\alpha_{2} \tau}\right) e^{-r \tau}\right] d \tau_{2}+ \\
\int_{\frac{1}{\alpha_{2}} \ln \left(\underline{\pi}_{N-1} / \pi_{0}\right)}^{\infty} \lambda_{2} e^{-\lambda_{2} \tau_{2}}\left[\int_{0}^{\frac{1}{\alpha_{2}} \ln \left(\underline{\pi}_{N-1} / \pi_{0}\right)} \pi_{0} e^{\alpha_{2} s} e^{-r s} d s+S\left(\frac{\pi_{0}}{\underline{\pi}_{N-1}}\right)^{\frac{r}{\alpha_{2}}}\right] d \tau_{2}
\end{array}\right\} .
\end{aligned}
$$

Because $\frac{p\left(\pi_{0} \mid \bar{\pi}\right)\left(\frac{\pi_{0}}{\underline{\pi}_{N-1}}\right)^{\frac{\lambda_{2}}{\alpha_{2}}}}{1-p\left(\pi_{0} \mid \bar{\pi}\right)+p\left(\pi_{0} \mid \bar{\pi}\right)\left(\frac{\pi_{0}}{\underline{\pi}_{N-1}}\right)^{\frac{\lambda_{2}}{\alpha_{2}}}}=p\left(\underline{\pi}_{N-1} \mid \bar{\pi}\right)$, algebraic manipulations yield that

$$
\begin{aligned}
\frac{\partial \underline{V}_{N-1}\left(\underline{\pi}_{N-1} \mid \pi_{0}, \bar{\pi}\right)}{\partial \underline{\pi}_{N-1}}= & \frac{\left(1-p\left(\pi_{0} \mid \bar{\pi}\right)+p\left(\pi_{0} \mid \bar{\pi}\right)\left(\frac{\pi_{0}}{\underline{\pi}_{N-1}}\right)^{\frac{\lambda_{2}}{\alpha_{2}}}\right)}{\alpha_{2} \underline{\pi}_{N-1}} \times \\
& {\left[\underline{\pi}_{N-1}-r S+p\left(\underline{\pi}_{N-1} \mid \bar{\pi}\right) \lambda_{2}\left(\bar{V}_{N}^{*}\left(\underline{\pi}_{N-1}\right)-S\right)\right]\left(\frac{\pi_{0}}{\underline{\pi}_{N-1}}\right)^{\frac{r}{\alpha_{2}}} . }
\end{aligned}
$$

Hence, the (candidate) maximizer $\underline{\pi}_{N-1}^{*}$ is given by the following first-order condition:

$$
r S=\underline{\pi}_{N-1}^{*}+p\left(\underline{\pi}_{N-1}^{*} \mid \bar{\pi}\right) \lambda_{2}\left(\bar{V}_{N}^{*}\left(\underline{\pi}_{N-1}^{*}\right)-S\right),
$$

where $\underline{\pi}_{N-1}^{*}>\bar{\pi}_{N}^{*}$ (since otherwise $\bar{V}_{N}^{*}\left(\underline{\pi}_{N-1}^{*}\right)=S$, and hence $\underline{\pi}_{N-1}^{*}=r S>\bar{\pi}_{N}^{*} \geq \underline{\pi}_{N-1}^{*}$, which is a contradiction). This implies that $\bar{\pi}_{N}^{*}<\underline{\pi}_{N-1}^{*}<r S$, and therefore the firm would never exit if another growth phase started. ${ }^{37}$

Using equation (35) yields that

$$
\begin{aligned}
\frac{\partial^{2} \underline{V}_{N-1}\left(\underline{\pi}_{N-1}^{*} \mid \pi_{0}, \bar{\pi}\right)}{\partial \underline{\pi}_{N-1}^{2}}= & \frac{\left(1-p\left(\pi_{0} \mid \bar{\pi}\right)+p\left(\pi_{0} \mid \bar{\pi}\right)\left(\frac{\pi_{0}}{\underline{\underline{\underline{\pi}}}_{N-1}^{*}}\right)^{\frac{\lambda_{2}}{\alpha_{2}}}\right)\left(\frac{\pi_{0}}{\underline{\pi}_{N-1}^{*}}\right)^{\frac{r}{\alpha_{2}}}}{\alpha_{2} \underline{\pi}_{N-1}^{*}} \times \\
& {\left[1+\lambda_{2} p\left(\underline{\pi}_{N-1}^{*} \mid \bar{\pi}\right) \frac{d \bar{V}_{N}^{*}\left(\underline{\pi}_{N-1}^{*}\right)}{d \pi_{0}}+\lambda_{2}\left(\bar{V}_{N}^{*}\left(\underline{\pi}_{N-1}^{*}\right)-S\right) \frac{\partial p\left(\underline{\pi}_{N-1}^{*} \mid \bar{\pi}\right)}{\partial \pi_{0}}\right], }
\end{aligned}
$$

so the facts that $\frac{\partial p\left(\underline{\pi}_{N-1}^{*} \mid \bar{\pi}\right)}{\partial \pi_{0}}=-\frac{\lambda_{2} p\left(\underline{\pi}_{N-1}^{*} \mid \bar{\pi}\right)\left(1-p\left(\underline{\pi}_{N-1}^{*} \mid \bar{\pi}\right)\right)}{\alpha_{2} \underline{\pi}_{N-1}^{*}}>0, \bar{V}_{N}^{*}\left(\underline{\pi}_{N-1}^{*}\right)>S$, and $\frac{d \bar{V}_{N}^{*}\left(\pi_{0}\right)}{d \pi_{0}}>0$ for any $\pi_{0}>\bar{\pi}_{N}^{*}$ imply that the global maximizer of $\underline{V}_{N-1}\left(\underline{\pi}_{N-1} \mid \pi_{0}, \bar{\pi}\right)$ is

\footnotetext{
${ }^{37}$ Suppose to the contrary that the firm found itself in a situation in which it would exit in the next growth phase. Then the continuity of the process implies that the firm should have already exited the market during the previous decline phase, which contradicts the hypothesis that the firm was active at the end of the downturn.
} 
indeed given by (35), at least if the solution to the maximization problem is interior.

Of course, the solution may not be interior and the firm may find it optimal to exit immediately once the market switches to decline. That is, for $\pi_{0}=\bar{\pi}$,

$$
\left.\frac{\partial \underline{V}_{N-1}\left(\underline{\pi}_{N-1} \mid \bar{\pi}, \bar{\pi}\right)}{\partial \underline{\pi}_{N-1}}\right|_{\underline{\pi}_{N-1}=\bar{\pi}} \geq 0
$$

might hold. This inequality is satisfied if and only if

$$
\bar{\pi}+p_{0} \lambda_{2}\left(\bar{V}_{N}^{*}(\bar{\pi})-S\right) \leq r S .
$$

Because the left hand side is increasing in $\bar{\pi}$, and exceeds $r S$ when $\bar{\pi}=r S$, there exists a unique state $\widehat{\pi}_{N-1}<r S$ such that the inequality holds with equality. Hence, condition (36) holds if and only if $\pi \leq \widehat{\pi}_{N-1}$, which means that the firm finds it optimal to disinvest right away whenever the market switches to decline at some level $\bar{\pi} \leq \widehat{\pi}_{N-1}$. Otherwise, the firm prefers waiting to disinvest and uses the threshold given by (35). Notice that the optimal investment threshold is an implicit function of the starting level $\bar{\pi}$, that is, we have that $\underline{\pi}_{N-1}^{*}(\bar{\pi})$. Hence, $\underline{\pi}_{N-1}^{*}(\bar{\pi})$ denotes the level below which the firm prefers exiting right away if the market is in the declining phase of the $N-1$ th cycle. For $\bar{\pi} \leq \widehat{\pi}_{N-1}, \underline{\pi}_{N-1}^{*}(\bar{\pi})=\bar{\pi}$, whereas $\underline{\pi}_{N-1}^{*}(\bar{\pi})$ is given by (35) for $\bar{\pi}>\widehat{\pi}_{N-1}$. In the latter case, the implicit function theorem and the fact that $\partial p\left(\underline{\pi}_{N-1}^{*} \mid \bar{\pi}\right) / \partial \bar{\pi}<0$ imply that

$$
\frac{d \underline{\pi}_{N-1}^{*}}{d \bar{\pi}}=-\frac{\lambda_{2}\left(\bar{V}_{N}^{*}\left(\underline{\pi}_{N-1}^{*}\right)-S\right) \frac{\partial p\left(\underline{\pi}_{N-1}^{*} \mid \bar{\pi}\right)}{\partial \bar{\pi}}}{1+\lambda_{2} p\left(\underline{\pi}_{N-1}^{*} \mid \bar{\pi}\right) \frac{d \bar{V}_{N}^{*}\left(\underline{\pi}_{N-1}^{*}\right)}{d \pi_{0}}+\lambda_{2}\left(\bar{V}_{N}^{*}\left(\underline{\pi}_{N-1}^{*}\right)-S\right) \frac{\partial p\left(\underline{\pi}_{N-1}^{*} \mid \bar{\pi}\right)}{\partial \pi_{0}}}>0 .
$$

Hence, the optimal disinvestment threshold increases with $\bar{\pi}$ for $\bar{\pi} \geq \widehat{\pi}_{N-1}$, with $\underline{\pi}_{N-1}^{*}(\bar{\pi}) \rightarrow$ $r S$ as $\bar{\pi} \rightarrow \infty$.

Let $\underline{V}_{N-1}^{*}\left(\pi_{0} \mid \bar{\pi}\right) \equiv \underline{V}_{N-1}\left(\underline{\pi}_{N-1}^{*}(\bar{\pi}) \mid \pi_{0}, \bar{\pi}\right)$, and consider the growing stage of cycle $N-1$. As usual, let $\pi_{0}$ denote the current state of the market, and denote the threshold at which the firm exits if the market is growing by $\bar{\pi}_{N-1}$. Then the firm's expected payoff is

$$
\begin{gathered}
\bar{V}_{N-1}\left(\bar{\pi}_{N-1} \mid \pi_{0}\right)=\int_{0}^{\frac{1}{\alpha_{1}} \ln \left(\bar{\pi}_{N-1} / \pi_{0}\right)} \lambda_{1} e^{-\lambda_{1} \tau_{1}}\left(\begin{array}{c}
\int_{0}^{\tau_{1}} \pi_{0} e^{\alpha_{1} s} e^{-r s} d s+ \\
\underline{V}_{N-1}^{*}\left(\pi_{0} e^{\alpha_{1} \tau_{1}} \mid \pi_{0} e^{\alpha_{1} \tau_{1}}\right) e^{-r \tau_{1}}
\end{array}\right) d \tau_{1}+ \\
\int_{\frac{1}{\alpha_{1}} \ln \left(\bar{\pi}_{N-1} / \pi_{0}\right)}^{\infty} \lambda_{1} e^{-\lambda_{1} \tau_{1}}\left(\int_{0}^{\frac{1}{\alpha_{1}} \ln \left(\bar{\pi}_{N-1} / \pi_{0}\right)} \pi_{0} e^{\alpha_{1} s} e^{-r s} d s+S\left(\frac{\pi_{0}}{\bar{\pi}_{N-1}}\right)^{\frac{r}{\alpha_{1}}}\right) d \tau_{1} .
\end{gathered}
$$


Noticing the similarity of this function and $\bar{V}_{N}\left(\bar{\pi}_{N} \mid \pi_{0}\right)$ yields that its derivative is equal to:

$$
\frac{\partial \bar{V}_{N-1}\left(\bar{\pi}_{N-1} \mid \pi_{0}\right)}{\partial \bar{\pi}_{N-1}}=\frac{\bar{\pi}_{N-1}-r S+\lambda_{1}\left(\underline{V}_{N-1}^{*}\left(\bar{\pi}_{N-1} \mid \bar{\pi}_{N-1}\right)-S\right)}{\alpha_{1} \bar{\pi}_{N-1}}\left(\frac{\pi_{0}}{\bar{\pi}_{N-1}}\right)^{\frac{r+\lambda_{1}}{\alpha_{1}}} .
$$

Consider a state $\bar{\pi}_{N-1}$ such that $\frac{\partial \bar{V}_{N-1}\left(\bar{\pi}_{N-1} \mid \pi_{0}\right)}{\partial \bar{\pi}_{N-1}}=0$ holds, and evaluate the second derivative at such state so as to get

$$
\frac{\partial^{2} \bar{V}_{N-1}\left(\bar{\pi}_{N-1} \mid \pi_{0}\right)}{\partial \bar{\pi}_{N-1}^{2}}=\frac{1}{\alpha_{1} \bar{\pi}_{N-1}}\left(\frac{\pi_{0}}{\bar{\pi}_{N-1}}\right)^{\frac{r+\lambda_{1}}{\alpha_{1}}}\left[1+\lambda_{1} \frac{d \underline{V}_{N-1}^{*}\left(\bar{\pi}_{N-1} \mid \bar{\pi}_{N-1}\right)}{d \pi_{0}}\right] .
$$

Because $\frac{d \underline{V}_{N-1}^{*}\left(\bar{\pi}_{N-1} \mid \bar{\pi}_{N-1}\right)}{d \pi_{0}} \geq 0$, it follows that the firm's objective function is strictly quasi-convex, and as a result, the firm must choose at state $\pi_{0}$ whether to exit immediately and seize $S$ or to wait until the market starts decaying and gain an expected payoff equal to

$$
\bar{V}_{N-1}\left(\infty \mid \pi_{0}\right)=\int_{0}^{\infty} \lambda_{1} e^{-\lambda_{1} \tau_{1}}\left(\int_{0}^{\tau_{1}} \pi_{0} e^{\alpha_{1} s} e^{-r s} d s+\underline{V}_{N-1}^{*}\left(\pi_{0} e^{\alpha_{1} \tau_{1}} \mid \pi_{0} e^{\alpha_{1} \tau_{1}}\right) e^{-r \tau_{1}}\right) d \tau_{1}
$$

Let $\bar{\pi}_{N-1}^{*}<r S$ be the unique level such that $\bar{V}_{N-1}\left(\infty \mid \pi_{0}\right)=S$ holds, and let $\bar{V}_{N-1}^{*}\left(\pi_{0}\right) \equiv$ $\max \left(S, \bar{V}_{N-1}\left(\infty \mid \pi_{0}\right)\right)$, that is,

$$
\bar{V}_{N-1}^{*}\left(\pi_{0}\right)=\left\{\begin{array}{cl}
\bar{V}_{N-1}\left(\infty \mid \pi_{0}\right) & \text { if } \pi_{0}>\bar{\pi}_{N-1}^{*} \\
S & \text { if } \pi_{0} \leq \bar{\pi}_{N-1}^{*}
\end{array}\right.
$$

To complete the proof, it only remains to show that the claim is true for $k \geq 2$ whenever it is true for $k-1$. So suppose that Claim 1 holds for cycle $N-k+1$, and let us derive the optimal disinvestment policy for cycle $N-k$. Although the proof follows verbatim the one for $k=1$ because of the recursive structure of the problem, we briefly go over each step for the sake of completeness. As for the declining phase, the firm's expected payoff is

$$
\begin{aligned}
& \underline{V}_{N-k}\left(\underline{\pi}_{N-k} \mid \pi_{0}, \bar{\pi}\right)=\left(1-p\left(\pi_{0} \mid \bar{\pi}\right)\right)\left[\int_{0}^{\frac{1}{\alpha_{2}} \ln \left(\underline{\underline{\pi}}_{N-k} / \pi_{0}\right)} \pi_{0} e^{\alpha_{2} s} e^{-r s} d s+S\left(\frac{\pi_{0}}{\underline{\pi}_{N-k}}\right)^{\frac{r}{\alpha_{2}}}\right]+ \\
& p\left(\pi_{0} \mid \bar{\pi}\right)\left[\begin{array}{c}
\int_{0}^{\frac{1}{\alpha_{2}} \ln \left(\underline{\pi}_{N-k} / \pi_{0}\right)} \lambda_{2} e^{-\lambda_{2} \tau_{2}}\left[\int_{0}^{\tau_{2}} \pi_{0} e^{\alpha_{2} s} e^{-r s} d s+\bar{V}_{N-k+1}^{*}\left(\pi_{0} e^{\alpha_{2} \tau_{2}}\right) e^{-r \tau_{2}}\right] d \tau_{2}+ \\
\int_{\frac{1}{\alpha_{2}} \ln \left(\underline{\pi}_{N-k} / \pi_{0}\right)}^{\infty} \lambda_{2} e^{-\lambda_{2} \tau_{2}}\left[\int_{0}^{\frac{1}{\alpha_{2}} \ln \left(\underline{\pi}_{N-k} / \pi_{0}\right)} \pi_{0} e^{\alpha_{2} s} e^{-r s} d s+S\left(\frac{\pi_{0}}{\underline{\pi}_{N-k}}\right)^{\frac{r}{\alpha_{2}}}\right] d \tau_{2}
\end{array}\right],
\end{aligned}
$$




$$
\begin{aligned}
\frac{\partial \underline{V}_{N-k}\left(\underline{\pi}_{N-k} \mid \pi_{0}, \bar{\pi}\right)}{\partial \underline{\pi}_{N-k}}= & \frac{\left(1-p\left(\pi_{0} \mid \bar{\pi}\right)+p\left(\pi_{0} \mid \bar{\pi}\right)\left(\frac{\pi_{0}}{\underline{\pi}_{N-k}}\right)^{\frac{\lambda_{2}}{\alpha_{2}}}\right)}{\alpha_{2} \underline{\pi}_{N-k}} \\
& {\left[\underline{\pi}_{N-k}-r S+p\left(\underline{\pi}_{N-k} \mid \bar{\pi}\right) \lambda_{2}\left(\bar{V}_{N-k+1}^{*}\left(\underline{\pi}_{N-k}\right)-S\right)\right]\left(\frac{\pi_{0}}{\underline{\pi}_{N-k}}\right)^{\frac{r}{\alpha_{2}}} . }
\end{aligned}
$$

Hence, in an interior solution we have that

$$
r S=\underline{\pi}_{N-k}^{*}+p\left(\underline{\pi}_{N-k}^{*} \mid \bar{\pi}\right) \lambda_{2}\left(\bar{V}_{N-k+1}^{*}\left(\underline{\pi}_{N-k}^{*}\right)-S\right),
$$

and it is standard to show that (34) (for the appropriate subscript) implies that $\underline{\pi}_{N-k}^{*}>$ $\bar{\pi}_{N-k+1}^{*}$. This yields that $\bar{\pi}_{N-k+1}^{*}<\underline{\pi}_{N-k}^{*}<r S$, and therefore the firm will never exit the market in the growing phase of cycle number $N-k+1$.

If the solution is not interior, that is, if for $\pi_{0}=\bar{\pi}$

$$
\left.\frac{\partial \underline{V}_{N-k}\left(\underline{\pi}_{N-k} \mid \pi_{0}, \bar{\pi}\right)}{\partial \underline{\pi}_{N-k}}\right|_{{\underline{\underline{\pi}_{N-k}}}_{\bar{\pi}}} \geq 0
$$

holds, then we must have that

$$
\bar{\pi}+p_{0} \lambda_{2}\left(\bar{V}_{N-k+1}^{*}(\bar{\pi})-S\right) \leq r S
$$

The left hand side is increasing in $\bar{\pi}$, and exceeds $r S$ when $\bar{\pi}=r S$, so there exists a unique state $\widehat{\pi}_{N-k}$ such that the inequality holds with equality. Hence, $\underline{\pi}_{N-k}^{*}(\bar{\pi})=\bar{\pi}$ for $\bar{\pi} \leq \widehat{\pi}_{N-k}$, while $\underline{\pi}_{N-k}^{*}(\bar{\pi})$ is given by (37) for $\bar{\pi}>\widehat{\pi}_{N-k}$. Again, it is easy to prove that $\frac{d \underline{\pi}_{N-k}^{*}}{d \bar{\pi}}>0$ for $\bar{\pi}>\widehat{\pi}_{N-k}$.

As for the growth stage, letting $\underline{V}_{N-k}^{*}\left(\pi_{0} \mid \bar{\pi}\right) \equiv \underline{V}_{N-k}\left(\underline{\pi}_{N-k}^{*}(\bar{\pi}) \mid \pi_{0}, \bar{\pi}\right)$ yields that the expected payoff of the firm at state $\pi_{0}$ if it chooses to exit at level $\bar{\pi}_{N-k}$ is

$$
\begin{aligned}
\bar{V}_{N-k}\left(\bar{\pi}_{N-k} \mid \pi_{0}\right)= & \int_{0}^{\frac{1}{\alpha_{1}} \ln \left(\bar{\pi}_{N-k} / \pi_{0}\right)} \lambda_{1} e^{-\lambda_{1} \tau_{1}}\left(\begin{array}{c}
\int_{0}^{\tau_{1}} \pi_{0} e^{\alpha_{1} s} e^{-r s} d s+ \\
\underline{V}_{N-k}^{*}\left(\pi_{0} e^{\alpha_{1} \tau_{1}} \mid \pi_{0} e^{\alpha_{1} \tau_{1}}\right) e^{-r \tau_{1}}
\end{array}\right) d \tau_{1}+ \\
& \int_{\frac{1}{\alpha_{1}} \ln \left(\bar{\pi}_{N-k} / \pi_{0}\right)}^{\infty} \lambda_{1} e^{-\lambda_{1} \tau_{1}}\left[\int_{0}^{\frac{1}{\alpha_{1}} \ln \left(\bar{\pi}_{N-k} / \pi_{0}\right)} \pi_{0} e^{\alpha_{1} s} e^{-r s} d s+S\left(\frac{\pi_{0}}{\bar{\pi}_{N-k}}\right)^{\frac{r}{\alpha_{1}}}\right] d \tau_{1} .
\end{aligned}
$$

This function can be easily shown to be strictly quasi-convex, ${ }^{38}$ so the firm must choose at

\footnotetext{
${ }^{38}$ Because $\underline{\pi}_{N-k}^{*}(\cdot)$ has a kink at $\widehat{\pi}_{N-k}$, it is reasonable to think that $\underline{V}_{N-k}^{*}\left(\pi_{0} \mid \pi_{0}\right)$ might not be differentiable at $\pi_{0}=\widehat{\pi}_{N-k}$, which would imply that $\bar{V}_{N-k}\left(\bar{\pi}_{N-k} \mid \pi_{0}\right)$ is not everywhere differentiable with
} 
state $\pi_{0}$ whether to exit immediately and seize $S$ or to wait until the market starts declining and gain an expected payoff equal to $\bar{V}_{N-k}\left(\infty \mid \pi_{0}\right)$. If $\bar{\pi}_{N-k}^{*}<r S$ denotes the unique level for which $\bar{V}_{N-k}\left(\infty \mid \pi_{0}\right)=S$ holds and we let $\bar{V}_{N-k}^{*}\left(\pi_{0}\right) \equiv \max \left(S, \bar{V}_{N-k}\left(\infty \mid \pi_{0}\right)\right)$, then the following holds:

$$
\bar{V}_{N-k}^{*}\left(\pi_{0}\right)=\left\{\begin{array}{cl}
\bar{V}_{N-k}\left(\infty \mid \pi_{0}\right) & \text { if } \pi_{0}>\bar{\pi}_{N-k}^{*} \\
S & \text { if } \pi_{0} \leq \bar{\pi}_{N-k}^{*}
\end{array} .\right.
$$

This shows that Claim 1 holds for cycle $N-k$, and hence completes the proof.

Proof of Proposition 6. Note that equation (5) holds if and only if

$$
\pi_{X}^{*} \alpha_{2} \rho_{2}\left(\beta_{2}-1\right)-\alpha_{2} \beta_{2} S=0
$$

is satisfied. Adding up $r S$ on both sides of this expression, and using the facts that $r-\alpha_{2} \beta_{2}=$ $\lambda_{2}\left(\delta_{1}-1\right)$ (see definition of $\delta_{1}$ in Theorem 3 for $\phi=1$ ) and $1+\lambda_{2} \gamma_{1}=\rho_{2}\left[\lambda_{2}\left(1-\delta_{1}\right)+r-\alpha_{2}\right]$ (so $1+\lambda_{2} \gamma_{1}=\alpha_{2} \rho_{2}\left(\beta_{2}-1\right)$ ) leads to the desired result.

Proof of Proposition 7. First, we prove that $\bar{V}_{X}^{*}\left(\pi_{X}^{*}\right)-\underline{V}_{X}^{*}\left(\pi_{X}^{*}\right)=\gamma_{1} \pi_{X}^{*}-\left(1-\delta_{1}\right) S>0$. By Proposition 6,

$$
\gamma_{1} \pi_{X}^{*}+\delta_{1} S-S=\frac{r S-\pi_{X}^{*}}{\lambda_{2}}
$$

so we simply have to show that $r>\frac{\beta_{2}}{\rho_{2}\left(\beta_{2}-1\right)}$ holds. To prove this, notice that

$$
\left(r+\lambda_{1}-\alpha_{1}\right)\left(r+\lambda_{2}-\alpha_{2}\right)-\lambda_{1} \lambda_{2}=\alpha_{1} \alpha_{2}\left(1-\beta_{1}\right)\left(1-\beta_{2}\right),
$$

respect to $\bar{\pi}_{N-k}$. To clear this doubt, we proceed to show that $\underline{V}_{N-k}^{*}\left(\pi_{0} \mid \pi_{0}\right) \equiv \underline{V}_{N-k}\left(\underline{\pi}_{N-k}^{*} \mid \pi_{0}, \pi_{0}\right)$ has a right-derivative at $\pi_{0}=\widehat{\pi}_{N-k}$ equal to 0 (since the left-derivative is 0 ). Because

$$
\begin{aligned}
\underline{V}_{N-k}^{*}\left(\pi_{0} \mid \pi_{0}\right)= & \left(1-p_{0}\right)\left[\int_{0}^{\frac{1}{\alpha_{2}} \ln \left(\underline{\pi}_{N-k}^{*} / \pi_{0}\right)} \pi_{0} e^{\alpha_{2} s} e^{-r s} d s+S\left(\frac{\pi_{0}}{\underline{\pi}_{N-k}^{*}}\right)^{\frac{r}{\alpha_{2}}}\right]+ \\
& p_{0}\left[\begin{array}{l}
\int_{0}^{\frac{1}{\alpha_{2}} \ln \left(\underline{\pi}_{N-k}^{*} / \pi_{0}\right)} \lambda_{2} e^{-\lambda_{2} \tau}\left[\int_{0}^{\tau} \pi_{0} e^{\alpha_{2} s} e^{-r s} d s+\bar{V}_{N-k+1}^{*}\left(\pi_{0} e^{\alpha_{2} \tau}\right) e^{-r \tau}\right] d \tau+ \\
\int_{\frac{1}{\alpha_{2}} \ln \left(\underline{\pi}_{N-k}^{*} / \pi_{0}\right)}^{\infty} \lambda_{2} e^{-\lambda_{2} \tau}\left[\int_{0}^{\frac{1}{\alpha_{2}} \ln \left(\underline{\pi}_{N-k}^{*} / \pi_{0}\right)} \pi_{0} e^{\alpha_{2} s} e^{-r s} d s+S\left(\frac{\pi_{0}}{\underline{\pi}_{N-k}^{*}}\right)^{\frac{r}{\pi_{2}}}\right] d \tau
\end{array}\right],
\end{aligned}
$$

the envelope theorem and the fact that $\underline{\pi}_{N-k}^{*}\left(\pi_{0}\right) \downarrow \widehat{\pi}_{N-k}$ as $\pi_{0} \downarrow \widehat{\pi}_{N-k}$ yield after canceling several terms that

$$
\left.\frac{d \underline{V}_{N-k}^{*}\left(\pi_{0} \mid \pi_{0}\right)}{d \pi_{0}}\right|_{\pi_{0} \downarrow \widehat{\pi}_{N-k}}=\left(1-p_{0}\right)\left(\frac{r S-\widehat{\pi}_{N-k}}{\alpha_{2} \widehat{\pi}_{N-k}}\right)+p_{0}\left(\frac{r S-\widehat{\pi}_{N-k}+\lambda_{2} S-\lambda_{2} \bar{V}_{N-k+1}^{*}\left(\widehat{\pi}_{N-k}\right)}{\alpha_{2} \widehat{\pi}_{N-k}}\right) .
$$

Since $\widehat{\pi}_{N-k}+p_{0} \lambda_{2}\left(\bar{V}_{N-k+1}^{*}\left(\widehat{\pi}_{N-k}\right)-S\right)=r S$ holds by definition of $\widehat{\pi}_{N-k}$, it follows that $\left.\frac{d \underline{V}_{N-k}^{*}\left(\pi_{0} \mid \pi_{0}\right)}{d \pi_{0}}\right|_{\pi_{0} \downarrow \widehat{\pi}_{N-k}}=0$, as desired. 
so we have that

$$
\rho_{2}=\frac{1}{r}\left[\frac{r\left(r+\lambda_{1}+\lambda_{2}\right)-r \alpha_{1}}{\alpha_{1} \alpha_{2}\left(1-\beta_{1}\right)\left(1-\beta_{2}\right)}\right]=\frac{1}{r}\left[\frac{\beta_{1} \beta_{2}-\frac{r}{\alpha_{2}}}{\left(1-\beta_{1}\right)\left(1-\beta_{2}\right)}\right],
$$

where the last equality follows because it holds that $\alpha_{1} \alpha_{2} \beta_{1} \beta_{2}=r\left(r+\lambda_{1}+\lambda_{2}\right)$. As a result, using the fact that $\alpha_{2} \beta_{2}-r=\lambda_{2}\left(1-\delta_{1}\right)$ (see definition of $\delta_{1}$ in Theorem 3 if $\phi=1$ ) yields that

$$
\frac{\left(\beta_{2}-1\right) \rho_{2} r-\beta_{2}}{\left(\beta_{2}-1\right) \rho_{2}}=\frac{\beta_{1} \beta_{2}-\frac{r}{\alpha_{2}}-\beta_{2}\left(\beta_{1}-1\right)}{\rho_{2}\left(\beta_{1}-1\right)\left(\beta_{2}-1\right)}=\frac{\lambda_{2}\left(1-\delta_{1}\right)}{\alpha_{2} \rho_{2}\left(\beta_{1}-1\right)\left(\beta_{2}-1\right)},
$$

whence it is clear that $\frac{\rho_{2} r\left(\beta_{2}-1\right)-\beta_{2}}{\rho_{2}\left(\beta_{2}-1\right)}=r-\frac{\beta_{2}}{\left(\beta_{2}-1\right) \rho_{2}}>0$, since $\delta_{1} \in(0,1), \beta_{1}>1$ and $\beta_{2}<0$.

Therefore, we must have that $\bar{V}_{X}^{*}\left(\pi_{X}^{*}\right)-\underline{V}_{X}^{*}\left(\pi_{X}^{*}\right)>0$, and to complete the proof it suffices to show that $\bar{V}_{X}^{*}\left(\pi_{0}\right)-\underline{V}_{X}^{*}\left(\pi_{0}\right)>\bar{V}_{X}^{*}\left(\pi_{X}^{*}\right)-\underline{V}_{X}^{*}\left(\pi_{X}^{*}\right)$ for $\pi_{0}>\pi_{X}^{*}$. To prove that $\bar{V}_{X}^{*}\left(\pi_{0}\right)-\underline{V}_{X}^{*}\left(\pi_{0}\right)$ is increasing for $\pi_{0}>\pi_{X}^{*}$, let $\pi_{0}>\pi_{X}^{*}$ and use the definition of $\gamma_{1}$ for $p_{0}=1$ so that

$$
\begin{aligned}
\bar{V}_{X}^{*}\left(\pi_{0}\right)-\underline{V}_{X}^{*}\left(\pi_{0}\right) & =\gamma_{1} \pi_{0}-\left(1-\delta_{1}\right) \underline{V}_{X}^{*}\left(\pi_{0}\right) \\
& =\frac{\left\{\rho_{2}\left[\lambda_{2}\left(1-\delta_{1}\right)+r-\alpha_{2}\right]-1\right\} \pi_{0}}{\lambda_{2}}-\left(1-\delta_{1}\right) \underline{V}_{X}^{*}\left(\pi_{0}\right) \\
& =\left(1-\delta_{1}\right)\left(\rho_{2} \pi_{0}-\underline{V}_{X}^{*}\left(\pi_{0}\right)\right)+\frac{\left[\rho_{2}\left(r-\alpha_{2}\right)-1\right] \pi_{0}}{\lambda_{2}} \\
& =\left(1-\delta_{1}\right)\left(\rho_{2} \pi_{X}^{*}-S\right)\left(\frac{\pi_{0}}{\pi_{X}^{*}}\right)^{\beta_{2}}+\frac{\left[\rho_{2}\left(r-\alpha_{2}\right)-1\right] \pi_{0}}{\lambda_{2}} \\
& =\frac{\left(1-\delta_{1}\right) S}{\beta_{2}-1}\left(\frac{\pi_{0}}{\pi_{X}^{*}}\right)^{\beta_{2}}+\frac{\left[\rho_{2}\left(r-\alpha_{2}\right)-1\right] \pi_{0}}{\lambda_{2}}
\end{aligned}
$$

where the last two equalities follow from Proposition 5. Note from expression (24) for $p_{0}=1$ (since $\bar{p} \equiv p\left(\pi_{0} \mid \bar{\pi}\right)$ equals 1 for $p_{0}=1$ ) that

$$
\rho_{2}\left(r-\alpha_{2}\right)-1=\frac{\lambda_{2}\left(\alpha_{1}-\alpha_{2}\right) \rho_{2}}{\left(\lambda_{1}+\lambda_{2}+r-\alpha_{1}\right)}
$$

SO

$$
\bar{V}_{X}^{*}\left(\pi_{0}\right)-\underline{V}_{X}^{*}\left(\pi_{0}\right)=\frac{\left(1-\delta_{1}\right) S}{\beta_{2}-1}\left(\frac{\pi_{X}^{*}}{\pi_{0}}\right)^{-\beta_{2}}+\frac{\left(\alpha_{1}-\alpha_{2}\right) \rho_{2} \pi_{0}}{\left(\lambda_{1}+\lambda_{2}+r-\alpha_{1}\right)} .
$$

Hence, $\bar{V}_{X}^{*}\left(\pi_{0}\right)-\underline{V}_{X}^{*}\left(\pi_{0}\right)$ is clearly increasing in $\pi_{0}$ (since $\delta_{1} \in(0,1)$ and $\beta_{2}<0$ ), which shows that $\bar{V}_{X}^{*}\left(\pi_{0}\right)-\underline{V}_{X}^{*}\left(\pi_{0}\right)>\bar{V}_{X}^{*}\left(\pi_{X}^{*}\right)-\underline{V}_{X}^{*}\left(\pi_{X}^{*}\right)$ for $\pi_{0}>\pi_{X}^{*}$. 


\section{References}

[1] Abel, A. B., and Janice C. Eberly (1996), "Optimal Investment with Costly Reversibility", Review of Economic Studies, 63(4): 581-593.

[2] Adner, R., and Peter Zemsky (2005), "Disruptive Technology and the Emergence of Competition", Rand Journal of Economics, 36(2): 229-254.

[3] Bagwell, K. and Robert Staiger (1997), "Collusion Over the Business Cycle", Rand Journal of Economics, 28(1): 82-106.

[4] Berman, J., and Janet Pfleeger (1997), "Which Industries are Sensitive to Business Cycles?", Monthly Labor Review, February: 19-25.

[5] Bernanke, B. S. (1983), "Irreversibility, Uncertainty, and Cyclical Investment", Quarterly Journal of Economics, 98(1): 85-106.

[6] Décamps, J-P., and Thomas Mariotti (2004), "Investment Timing and Learning Externalities", Journal of Economic Theory, 118(1): 80-102.

[7] Dixit, A. K. (1989), "Entry and Exit Decisions under Uncertainty", Journal of Political Economy, 97(3): 620-638.

[8] Dixit, A. K., and Robert S. Pindyck (1994), Investment under Uncertainty, New Jersey: Princeton University Press.

[9] Driffill, J., Marzia Raybaudi, and Martin Sola (2003), "Investment under Uncertainty with Stochastically Switching Profit Streams: Entry and Exit over the Business Cycle", Studies in Nonlinear Dynamics \& Econometrics, 7(1): 1-38.

[10] Guo, X., Jianjun Miao, and Erwan Morellec (2005), "Irreversible Investment with Regime Shifts", Journal of Economic Theory, 122(1): 37-59.

[11] Keller, G., Sven Rady, and Martin Cripps (2005), "Strategic Experimentation with Exponential Bandits", Econometrica, 73(1): 39-68.

[12] Lucas, R. (1971), "Optimal Management of a Research and Development Project", Management Science, 17(11): 679-697.

[13] McAfee, R. P. (2002), Competitive Solutions, New Jersey: Princeton University Press.

[14] McDonald, R., and Daniel Siegel (1986), "The Value of Waiting to Invest", Quarterly Journal of Economics, 101(4): 707-728. 
[15] Miao, J., and Neng Wang (2006), "Learning, Investment, and Entrepreneurial Survival: A Real Options Approach", mimeo, Boston University.

[16] Moscarini, G., and Francesco Squintani (2004), "Competitive Experimentation with Private Information", mimeo, Yale University.

[17] Pindyck, R. S. (1991), "Irreversibility, Uncertainty, and Investment", Journal of Economic Literature, 29(3): 1110-1148.

[18] Riedel, F., and Xia Su (2007), "On Irreversible Investment", mimeo, University of Bonn. 